\title{
Membrane locking in discrete shell theories
}

Dissertation

zur Erlangung des mathematisch-naturwissenschaftlichen Doktorgrades Doctor rerum naturalium

der Georg-August-Universität Göttingen

vorgelegt von

Alessio Quaglino

aus Cantù

Göttingen 2012 
D7

Referent: Prof. Max Wardetzky

Koreferent: Prof. Gert Lube

Tag der mündlichen Prüfung: 11.05.2012 


\section{Contents}

List of abbreviations

1. Overview 1

1.1. An overview of this work . . . . . . . . . . . . . 1

1.2. An overview of locking . . . . . . . . . . . . . . . 2

2. Monodimensional warm-up $\mathbf{5}$

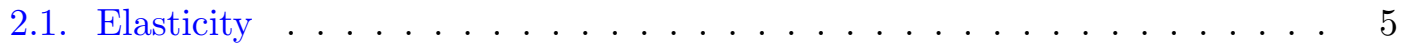

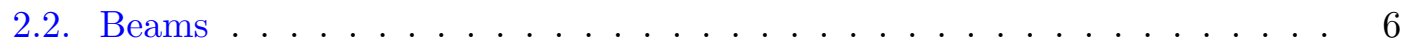

2.3. Rods . . . . . . . . . . . . . . . . . . . . . . . . 11

2.4. A first encounter with locking . . . . . . . . . . . . . . . . . . 14

2.5. Discrete rods . . . . . . . . . . . . . . . . . . . 15

2.6. Adaptivity . . . . . . . . . . . . . . . . . . . . . 17

2.7. Wrinkling . . . . . . . . . . . . . . . . . 20

3. Smooth surfaces 23

3.1. Geometry of shells . . . . . . . . . . . . . . . . . 23

3.2. Plates . . . . . . . . . . . . . . . . 26

3.3. Physics of shells . . . . . . . . . . . . . . . . . . . . 30

3.4. Membranes . . . . . . . . . . . . . . . . . . 33

3.5. Developable surfaces . . . . . . . . . . . . . . . . . . . 35

4. Discrete surfaces $\quad 39$

4.1. Discrete shell theories . . . . . . . . . . . . . . . . . . . 39

4.2. Discrete curvature in plates . . . . . . . . . . . . . . . . 46

4.3. Discrete membranes . . . . . . . . . . . . . . . . . . . 48

4.4. Discrete developable surfaces . . . . . . . . . . . . . . . . . . 49

4.5. Membrane locking . . . . . . . . . . . . . . . . 53

5. Adaptive surfaces $\quad \mathbf{5 9}$

5.1. Edgepoints . . . . . . . . . . . . . . . . . . . 59

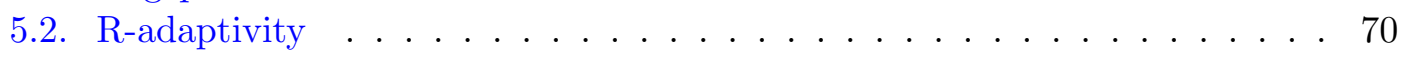

5.3. Dynamics . . . . . . . . . . . . . . . . . . 75

5.4. Energy-based combinatorics . . . . . . . . . . . . . . . 80

$\begin{array}{lr}\text { 6. Mimetic surfaces } & \mathbf{8 3}\end{array}$

6.1. Physics of locking . . . . . . . . . . . . . . . 83 
Contents

6.2. Kinematics . . . . . . . . . . . . . . . . . 87

6.3. Energy . . . . . . . . . . . . . . . . . . . . . . . . 92

6.4. Numerical experiments . . . . . . . . . . . . . . . . . . . . . 97

$\begin{array}{ll}\text { 7. Conclusions } & 105\end{array}$

$\begin{array}{ll}\text { A. Derivatives } & 107\end{array}$

$\begin{array}{lr}\text { B. Implementation } & 113\end{array}$

$\begin{array}{lr}\text { Acknowledgements } & 117\end{array}$

$\begin{array}{lr}\text { Bibliography } & 119\end{array}$ 


\section{List of abbreviations}

$\mathbb{P}^{r} \quad$ Polynomial of degree $r$

AL Augmented Lagrangian, 16

$\mathrm{CCH}$ Constant curvature hinge (discrete curvature), 42

CRT Crouzeix-Raviart triangle (discrete membrane), 41

CST Constant strain triangle (discrete membrane), 41

DDG Discrete differential geometry, 1

DKT Discrete Kirchhoff triangle (discrete curvature), 47

DOF Degree of freedom, 2

dST Discrete linear strain triangle (novel discrete membrane), 94

EAS Enhanced assumed strain, 15

EB Euler-Bernoulli beam theory, 8

EBT English-Bridson triangle (discrete developable surface), 51

ELT Eulerian-Lagrangian triangle (novel discrete membrane), 59

FEM Finite element method, 1

KL Kirchhoff-Love plate theory, 27

LCT Linear curvature triangle (novel discrete curvature), 95

LMT Linear membrane triangle (novel discrete membrane), 92

MSO Midedge shape operator (discrete curvature), 43

PDE Partial differential equation, 2

PPM Push-pull failure mode, 86

RM Reissner-Mindlin plate theory, 28

SSP Simply supported plate, 86 



\section{Overview}

This work is concerned with the study of thin structures in Computational Mechanics. This field is particularly interesting, since together with traditional finite elements methods (FEM), the last years have seen the development of a new approach, called discrete differential geometry (DDG).

The idea of FEM is to approximate smooth solutions using polynomials, providing error estimates that establish convergence in the limit of mesh refinement. The natural language of this field has been found in the formalism of functional analysis.

On the contrary, DDG considers discrete entities, e.g., the mesh, as the only physical system to be studied and discrete theories are being formulated from first principles. In particular, DDG is concerned with the preservation of smooth properties that break down in the discrete setting with FEM.

While the core of traditional FEM is based on function interpolation, usually in Hilbert spaces, discrete theories have an intrinsic physical interpretation, independently from the smooth solutions they converge to. This approach is related to flexible multibody dynamics and finite volumes.

In this work, we focus on the phenomenon of membrane locking, which produces a severe artificial rigidity in discrete thin structures. In the case of FEM, locking arises from a poor choice of finite subspaces where to look for solutions, while in the DDG case, it arises from arbitrary definitions of discrete geometric quantities.

In particular, we underline that a given mesh, or a given finite subspace, are not the physical system of interest, but a representation of it, out of infinitely many. In this work, we use this observation and combine tools from FEM and DDG, in order to build a novel discrete shell theory, free of membrane locking.

\subsection{An overview of this work}

In Chapter 2, we present a self-contained summary of the fundamental concepts of the thesis, for 1D bodies. Although membrane locking is not an issue here, we will introduce some of the challenges stemming from the techniques used to tackle locking in 2D.

In Chapter 3 and 4, we present a novel overview of known smooth and discrete theories of surfaces, attempting an unified presentation of topics from applied mathematics, engineering, and computer graphics. In particular, we remark that thin surfaces exhibit very different asymptotic regimes, whose preservation in the discrete setting is often overlooked, leading to failure modes and membrane locking.

In Chapter 5, we present a variational approach to tackle locking. We start by introducing a novel FE, having degrees of freedom (DOFs) in the undeformed configuration, 


\section{Overview}

which turned out to be a special case of $r$-adaptivity. We illustrate issues regarding degenerate triangles and dynamic simulations, discussing the drawbacks which ultimately led us to abandon this method. We conclude by discussing energy-based $h$-adaptivity to introduce the following chapter.

In Chapter 6, we present our main contribution, which is tailored not to approximate the smooth shell equations, but to mimic the asymptotic behavior of smooth surfaces, inside each triangle of the mesh. This will lead to a novel discrete shell theory, having linear strains and curvatures, which is remarkably free of locking and spurious modes, while being computationally cheaper than high-order FE.

In Appendix A, we provide the explicit calculation of gradients and hessians with respect to undeformed DOFs, for some of the FE considered in Chapter 5.

In Appendix B, we provide details on the implementation of the models described in the thesis. In particular, we sketch the structure of MEshopt, a $\mathrm{C}++$ library for cloth simulations and geometry processing we contributed to develop since 2009.

\subsection{An overview of locking}

A large class of problems in the theory of PDE, is concerned with the solution $u^{t}$ of

$$
\inf _{u \in X} \frac{1}{2}\left(a(u)+\frac{1}{t^{2}} b(u)\right)-(f, u),
$$

where $X$ is a Sobolev space, $t$ is a (small) physical parameter, and $f \in X^{\prime}$ is a given load in the dual space $X^{\prime}$. Let $u_{h}^{t} \in X_{h}$ be the approximation of $u^{t}$, computed using a certain discretization $X_{h}$ on a mesh with resolution $h$. Our discussion will focus on finding a robust discretization for the above system, in accordance with [15]:

Definition 1.1. (Robustness) Given $\delta>0$, there is $\gamma>0$ such that $\left\|u_{h}^{t}-u^{t}\right\|<\delta$ for $h \in(0, \gamma)$ and $t \in\left(0, t_{0}\right)$.

Conversely, when $\gamma=\gamma(t) \rightarrow 0$ for $t \rightarrow 0$ we say that a discretization exhibits locking. In particular, we are interested in the case $b(u)=\|c(u)\|^{2}=\langle c(u), c(u)\rangle$, which can be formulated in two different ways:

- Via penalty methods:

$$
\inf _{u \in X} \frac{1}{2}\left(a(u)+\frac{1}{t^{2}}\|c(u)\|^{2}\right)-(f, u)
$$

leading to an ill-conditioned but convex problem, meaning that any conforming (i.e. $\left.X_{h} \subset X\right)$ discretization is convergent, but not necessarily robust.

- Via Lagrange multipliers:

$$
\inf _{u \in X} \sup _{\sigma \in Q} \frac{1}{2}\left(a(u)-t^{2}\|\sigma\|^{2}\right)+\langle\sigma, c(u)\rangle-(f, u),
$$

leading to a well-conditioned saddle-point problem, which however is well-posed only if the choice of discrete spaces $\left(X_{h}, Q_{h}\right)$ is consistent. 


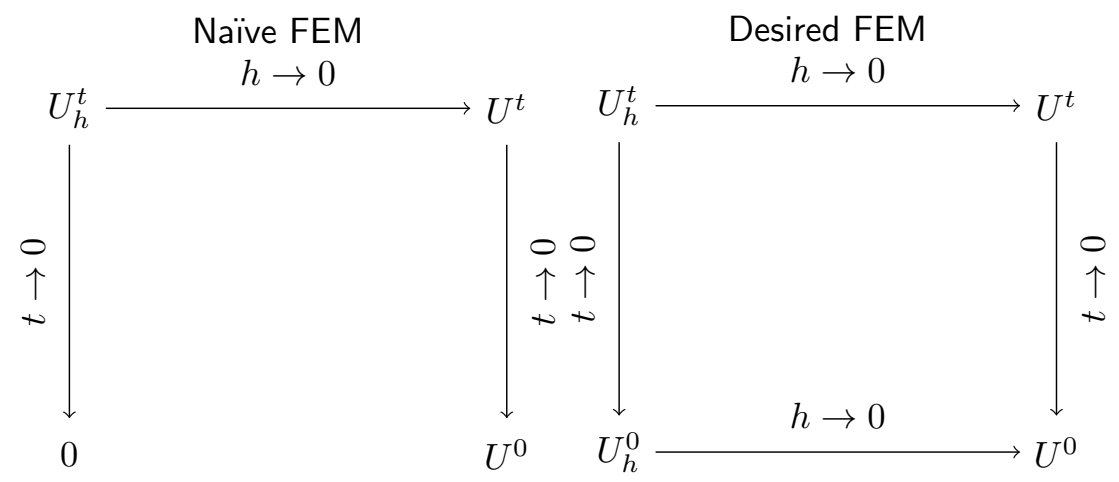

Figure 1.1.: Comparison between locking (on the left) and robust FE (on the right) [27].

In this context, for $t \rightarrow 0$, we will refer to $c(u)=0$ as a constraint. However, as will be explained in Chapter 3, (1.1) reduces to a constrained problem only under certain assumptions regarding $X$ and $f$. Throughout this chapter, we assume this to be the case, since locking appears in this scenario. However, complicated phenomena such as boundary layers, can appear if these assumptions are not satisfied [40].

We sketched in Fig. 1.1 a typical behavior of a locking-inducing discretization $X_{h}$. In order to avoid locking, (1.2) and (1.2) are normally employed with reduced integration or a lower-order space $Q_{h} \subset Q$, respectively. By doing so, one may easily incur in the mistake of allowing too much freedom, such that uncontrolled, or even unbounded, violations of the constraint are possible. Normally, there are two different approaches to avoid such situations:

- Build the discrete constraint such that $\operatorname{Ker}\left(c_{h}\right) \subset \operatorname{Ker}(c)$, where $c_{h}$ is, in general, not the restriction on $X_{h}$ of $c$. This is similar to looking for reduced coordinates.

- Choose a larger space, thus relaxing the constraint, under the condition that $a(u)$, possibly including an appropriate perturbation, must be convex there. This is linked to reduced integration and stabilization techniques.

In this work, we will see that in the case of thin structures, the first strategy often leads to locking, while the second one may fail when $X$ and $f$ are such that $c(u) \neq 0$ in the asymptotic limit $t \rightarrow 0$. There is a wide literature regarding the analysis of the approximations of linearly constrained problems of the form $c(u)=B u$ [13], but no systematic cure for locking.

In order to perform a first analysis of the problem, suppose that $b(u)=0$ and $a(u)$ is quadratic in $u$, i.e., it can be expressed using a bilinear form $a_{0}: X \times X \rightarrow \mathbb{R}$. Then, we have the following classical result.

Theorem 1.1. (Lax-Milgram) Let $X$ be a Hilbert space and let $a_{0}: X \times X \rightarrow \mathbb{R}$ be an elliptic $a_{0}(v, v) \geq \alpha_{0}\|v\|^{2}$ and continuous $a_{0}(u, v) \leq C\|u\|\|v\| \forall u, v, \in X$ bilinear form. 


\section{Overview}

Then, for any $f \in X^{\prime}$ there exists a unique minimizer of:

$$
E_{0}(u)=\frac{1}{2} a_{0}(u, v)-(f, v) .
$$

For a proof, see [12]. Moreover, the following stability result provides an estimate of the ill-conditioning of the approximation in a given discrete space.

Theorem 1.2. (Cea) Let $a_{0}$ be elliptic on $X \subset H^{m}$. If $u$ and $u_{h}$ are the solution of the variational problem in $X$ and $X_{h} \subset X$, then:

$$
\left\|u-u_{h}\right\| \leq \frac{C}{\alpha_{0}} \inf _{v_{h} \in X_{h}}\left\|u-v_{h}\right\|_{m} .
$$

Now, let $a_{0}$ be continuous, symmetric, coercive with $a_{0}(v, v) \geq \alpha_{0}\|v\|^{2}$, and $b(u)=$ $(B u, B u)$, where $B: X \rightarrow L^{2}(\Omega)$ a continuous linear mapping. In addition, let $t$ be a parameter $0 \leq t \leq 1$. Given $f \in X^{\prime}$, we seek a minimizer $u:=u_{t} \in X$ of the functional

$$
E\left(u^{t}\right)=\frac{1}{2} a_{0}\left(u^{t}, u^{t}\right)+\frac{1}{2 t^{2}}\left(B u^{t}, B u^{t}\right)-\left\langle f, u^{t}\right\rangle .
$$

The existence and uniqueness are guaranteed by the coercivity of $a(u, v):=a_{0}(u, v)+$ $t^{-2}(B u, B v)$. Generally, $B$ has nontrivial kernel and $\operatorname{dim} \operatorname{ker} B=\infty$, while the locking effect occurs when $X_{h} \cap \operatorname{ker} B=\{0\}$. We now give lower and upper bound estimates of the solution, more details can be found in [12].

Lemma 1.3. (Lower bound) Let $u_{0}$ such that $B u_{0}=0$ and $d:=\left\langle f, u_{0}\right\rangle>0$. Then, the following holds:

$$
\left\|u^{t}\right\| \geq\|f\|_{X^{\prime}}^{-1} \frac{1}{2} d, \quad \forall t>0 .
$$

Lemma 1.4. (Upper bound) Let $\left\|B v_{h}\right\| \geq C(h)\left\|v_{h}\right\|_{X}$ for all $v_{h} \in X_{h}$. Then, it follows:

$$
\left\|u_{h}^{t}\right\| \leq \alpha^{-1}\|f\|_{X^{\prime}} \leq t^{2} C(h)^{-2}\|f\|_{X^{\prime}} .
$$

For a small parameter $t,(1.8)$ gives a solution which is too small in contrast to (1.7). This is what engineers recognize as locking. The convergence cannot be uniform in $t$ as $h \rightarrow 0$. On the other hand, a finite element method is called robust for a problem with a small parameter $t$, if the convergence is uniform in $t$.

Remark. In the limit $t \rightarrow 0$, a sufficient condition for the state variable $u$ to exist uniquely, is $a(u)$ being convex on $\operatorname{Ker}(c)$ [13]. However, for nonlinearly constrained FE, convexity over $X$ does not imply convexity over $\operatorname{Ker}(c) \subseteq X$, since the set $\operatorname{Ker}(c)$ is not in general a subspace of $X$.

Therefore, the above canonical Sobolev approach, usually applied to the analysis of the linearized problem, must be dropped for the full nonlinear case, including bifurcations and material instabilities [53]. 


\section{Monodimensional warm-up}

In this chapter, we consider the deformation of an elastic body $\mathcal{S} \subset \mathbb{R}$ embedded in $\mathbb{R}^{2}$, in order to illustrate the following concepts:

1. Thick vs thin assumptions.

2. Small vs large deflections.

3. Coordinate vs objective representations.

4. Locking vs robust approximations.

5. Low-order vs higher-order methods.

The goal is to understand how to devise a robust and low-order discretization for large deformations of thin structures. We will discuss our preference towards intrinsic representations in order to gain precious intuition of the physics behind the model. At the end, we present an informal discussion of the two main techniques on which we will rely in the next chapters to defeat locking:

- R-adaptivity as a tool to find the optimal embedding of a mesh with given connectivity.

- Wrinkling as a concept to formulate discrete kinematic theories reproducing the correct physical regime under compressive and tensional loads.

\subsection{Elasticity}

We start this chapter by introducing the fundamental concepts of continuum mechanics. An elastic body $\mathcal{S}$ is a collection of material points $X$, which we consider to be embedded in $\mathbb{R}^{2}$, through a mapping $\phi: \mathcal{S} \rightarrow \mathbb{R}^{2}$, called a configuration of $\mathcal{S}$. In order to be physically realizable, $\phi$ needs to be sufficiently smooth, orientation preserving, and invertible. Points in the image of $\phi$ are denoted with $\mathbf{x} \in \mathbb{R}^{2}$ and are called spatial points. Spatial and material points are related by the kinematic relations

$$
\begin{aligned}
& \mathbf{x}=\phi(X), \\
& \overline{\mathbf{x}}=\bar{\phi}(X),
\end{aligned}
$$




\section{Monodimensional warm-up}

where the bar denotes the undeformed configuration of $\mathcal{S}$. The words configuration and deformation are thus synonymous. The geometric measure of the deformation is the pullback of the metric tensor, called the deformation tensor, which for a curve parametrized as $\mathbf{x}(t)=(x(t), y(t))$, becomes the scalar quantity

$$
C(\phi)=\nabla \mathbf{x}^{T} \nabla \mathbf{x}=\left(\frac{\partial x}{\partial t}\right)^{2}+\left(\frac{\partial y}{\partial t}\right)^{2}
$$

which is the infinitesimal length of the curve described by $\phi$. The geometric measure of the deformation is called the strain tensor, which in this case is the scalar quantity

$$
\epsilon(\phi)=\frac{1}{2}(C-\bar{C}) .
$$

In the case when $\bar{C}=I d$, the strain is small, and the quantity $x-\bar{x}$ contains only infinitesimal rotations, the following approximation is valid

$$
\epsilon \approx \frac{1}{2}\left(C+C^{T}\right)-I d
$$

The physical measure of the deformation is an energy obtained from the weighted Frobenius norm of the strain tensor

$$
U(\phi)=\int_{\mathcal{S}}\|\epsilon\|_{M}^{2} d \bar{x}=\int_{\mathcal{S}} \epsilon: E: \epsilon d \bar{x},
$$

where $E$ is a fourth order tensor containing the material properties, : denotes the operation $A: B=\sum_{i j} A_{k l i j} B_{i j}$. By introducing the stress tensor $\sigma:=E: \epsilon$, which measures the internal forces acting in the body, the energy can be written in the general form

$$
U(\phi)=\int_{\mathcal{S}} \sigma: \epsilon d \bar{x} .
$$

Given the above ingredients, the task is to find the deformation $\phi$ which minimizes the elastic energy, i.e., to solve the problem

$$
\inf _{\phi \in X(\mathcal{S})} U(\phi),
$$

where $X(\mathcal{S})$ is in general a Banach space.

\subsection{Beams}

A beam is a 1-dimensional straight structural element that is capable of withstanding load primarily by resisting bending. Prevailing consensus is that Galileo Galilei made the first attempts at developing a theory of beams, but recent studies argue that Leonardo da Vinci was the first to make the crucial observations. Unfortunately, Da Vinci lacked Hooke's law and calculus to complete the theory, whereas Galileo was held back by an incorrect assumption he made, thus beam theory had to wait until the $18^{\text {th }}$ century to appear in the current form. 


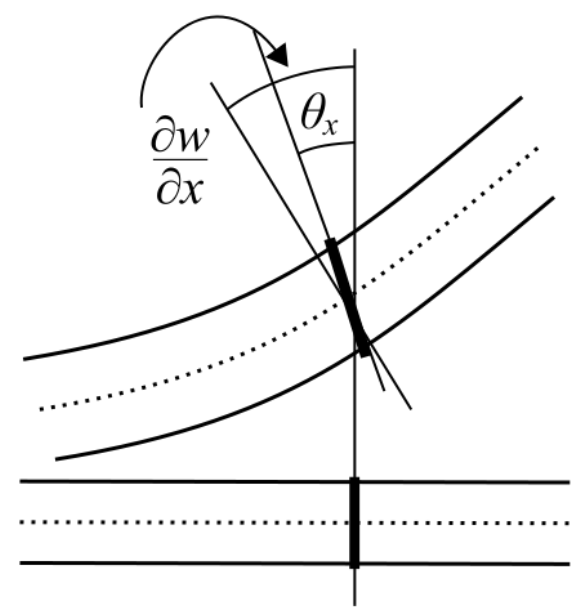

Figure 2.1.: Deformation of a Timoshenko beam. The normal rotates by an amount $\theta_{x}=\theta(x)$ which is not equal to $\partial w / \partial x$.

\subsubsection{Kinematic assumptions}

Let each material point $p \in \mathcal{S}$ be embedded in $\mathbb{R}^{2}$ with undeformed and deformed coordinates given by $\overline{\mathbf{x}}(p)=(x, 0)$ and $\mathbf{x}(p)=\overline{\mathbf{x}}(p)+\mathbf{u}(p)$, where $\mathbf{u}=\left(u_{x}, u_{z}\right) \in \mathbb{R}^{2}$ is the displacement vector of the point. In beam theory, the displacements are assumed to be small so that they can be described by the first-order approximation

$$
\begin{aligned}
& u_{x}(x, z)=u_{0}(x)-z \theta(x), \\
& u_{z}(x, z)=w(x)
\end{aligned}
$$

where $\theta$ is the angle of rotation of the normal to the mid-surface of the beam, $w$ is the displacement of the mid-surface in the z-direction, and we set $u_{0}=0$ for simplicity. Apart from its coordinate system, a beam is characterized by the following physical properties:

- The cross section area $A(x)$ and the length $L$.

- The elastic modulus $E(x)$, which determines the elastic response of the beam.

- The shear modulus $G(x)$, which penalizes the deviation of $\theta$ from $\frac{\partial w}{\partial x}$.

- The second moment of area $I(x)=\int z^{2} d A$, calculated with respect to the centroidal axis perpendicular to the applied loading.

- The applied force per unit length $q(x)$, known as distributed load. 


\section{Monodimensional warm-up}

\section{Timoshenko beam}

The Timoshenko beam theory was developed by Ukrainian-born scientist Stephen Timoshenko in the beginning of the 20th century [54]. As shown in figure 2.1, the Timoshenko beam theory accounts for shear deformation

$$
\theta \neq \frac{\partial w}{\partial x},
$$

making it suitable for describing the behaviour of thick and composite beams. This effectively lowers the stiffness of the beam, yielding a larger deflection under a static load for a given set of boundary conditions. If the shear modulus of the beam material approaches infinity, Timoshenko beam theory converges towards the Euler-Bernoulli beam theory.

\section{Euler-Bernoulli (EB) beam}

The Bernoulli beam is named after Jacob Bernoulli, who made the first significant discoveries, whereas Leonhard Euler and Daniel Bernoulli were the first to put together a useful theory around 1750 . Unfortunately, science and engineering were generally seen as very distinct fields at the time, and there was considerable doubt that a mathematical product of academia could be trusted for practical safety applications. Bridges and buildings continued to be designed by precedent methods until the late 19th century, when the Eiffel Tower demonstrated the validity of the theory on large scales.

This theory is a special case of the Timoshenko beam, formulated with the special assumption

$$
\theta=\frac{\partial w}{\partial x} .
$$

Its importance lies in the fact that in the thin limit the two theories behave identically, and equation (2.12) becomes a constraint in the Timoshenko theory. Therefore, the EB theory is a reduced coordinate representation which becomes available in the case of thin beams. Notice that, in general, such a representation is not explicitly available for nonlinearly constrained systems.

\subsubsection{Strains}

From the kinematics defined in (2.9) - (2.10), the linearized strains of the beam are readily obtained

$$
\begin{aligned}
\varepsilon_{x x} & =\frac{\partial u_{x}}{\partial x}=-z \frac{\partial \theta}{\partial x} \\
\varepsilon_{x z} & =\frac{1}{2}\left(\frac{\partial u_{x}}{\partial z}+\frac{\partial u_{z}}{\partial x}\right)=\frac{1}{2}\left(-\theta+\frac{\partial w}{\partial x}\right) .
\end{aligned}
$$

For the EB beam theory, we obtain $\varepsilon_{x z}=0$ and

$$
\varepsilon_{x x}=-z \frac{\partial^{2} w}{\partial x^{2}} .
$$




\subsubsection{Energy}

The Hooke's law stresses for the strains defined above are

$$
\begin{aligned}
\sigma_{x x} & =E \varepsilon_{x x}=-z E \frac{\partial \theta}{\partial x}, \\
\sigma_{s} & =2 G \varepsilon_{x z}=\kappa G\left(-\theta+\frac{\partial w}{\partial x}\right),
\end{aligned}
$$

where the correction factor $\kappa$ takes in account the fact that the actual shear strain in the beam is not constant over the cross section. Normally, $\kappa=5 / 6$ for a rectangular section. The total energy of the beam is given by the difference between the internal energy $U$ and the external work $W$ as

$$
\begin{aligned}
U-W & =\int_{L}\left[\int_{A}\left(\sigma_{x x} \varepsilon_{x x}+2 \sigma_{s} \varepsilon_{x z}\right) \mathrm{d} A-q w\right] \mathrm{d} L \\
& =\int_{L}\left[\int_{A}\left(E\left(z \frac{\partial \theta}{\partial x}\right)^{2}+\kappa G\left(\frac{\partial w}{\partial x}-\theta\right)^{2}\right) \mathrm{d} A-q w\right] \mathrm{d} L \\
& =\int_{L}\left[E I\left(\frac{\partial \theta}{\partial x}\right)^{2}+\kappa A G\left(\frac{\partial w}{\partial x}-\theta\right)^{2}-q w\right] \mathrm{d} L,
\end{aligned}
$$

where we notice that the second term is a penalty method for enforcing condition (2.12), which is built-in in the EB model. Assuming constant E, I, $A$ and taking variations of $U$, the governing equations for the beam may be expressed as [54]

$$
\begin{aligned}
\frac{\partial w}{\partial x}+\frac{1}{\kappa A G} \frac{\partial}{\partial x}\left(E I \frac{\partial \theta}{\partial x}\right) & =\theta \\
\frac{\partial^{2}}{\partial x^{2}}\left(E I \frac{\partial \theta}{\partial x}\right) & =q .
\end{aligned}
$$

Combining the Timoshenko equations and assuming a homogeneous beam of constant cross-section, gives

$$
E I \frac{\mathrm{d}^{4} w}{\mathrm{~d} x^{4}}=q(x)-\frac{E I}{k A G} \frac{\mathrm{d}^{2} q}{\mathrm{~d} x^{2}} .
$$

The Timoshenko beam theory for the static case is equivalent to the Euler-Bernoulli theory when the last term above is neglected, an approximation that is valid when

$$
\frac{E I}{\kappa L^{2} A G} \ll 1
$$

which yields the biharmonic equation

$$
E I \frac{\mathrm{d}^{4} w}{\mathrm{~d} x^{4}}=q .
$$

This equation is widely used in engineering practice. Tabulated expressions for the deflection $w$ for common beam configurations can be found in engineering handbooks. For 


\section{Monodimensional warm-up}

more complicated situations the deflection can be determined by solving it using techniques such as the "slope deflection method", "moment distribution method", "moment area method, "conjugate beam method", "the principle of virtual work", "direct integration", "Castigliano's method", "Macaulay's method" or the "direct stiffness method".

In particular, engineers are concerned with the computations of the following quantities for given loads:

- $M=-E I \frac{\mathrm{d}^{2} w}{\mathrm{~d} x^{2}}$ is the bending moment in the beam.

- $S=-\frac{\mathrm{d}}{\mathrm{d} x}\left(E I \frac{\mathrm{d}^{2} w}{\mathrm{~d} x^{2}}\right)$ is the shear force in the beam.

Remark. The sign convention has been chosen so the coordinate system is right handed. Forces acting in the positive $x$ and $z$ directions are assumed positive. The sign of the bending moment is chosen so that a positive value leads to a tensile stress at the bottom cords. The sign of the shear force has been chosen such that it matches the sign of the bending moment.

\subsubsection{Von Karman beam}

The original Euler-Bernoulli theory is valid only for infinitesimal strains and small rotations. The theory can be extended to problems involving moderately large rotations provided that the strain remains small by using the von Karman strains, which are found by discarding higher-order in-plane terms in finite Lagrange-Green strain. By considering the general case $u_{0} \neq 0$ in $(2.9)-(2.10)$ and unshearable rods, the strain is

$$
\epsilon_{x x}=\frac{d u_{0}}{d x}-\frac{d^{2} w}{d x^{2}}+\frac{1}{2}\left(\frac{d w}{d x}\right)^{2} .
$$

To close the system of equations we need the constitutive equations that relate stresses

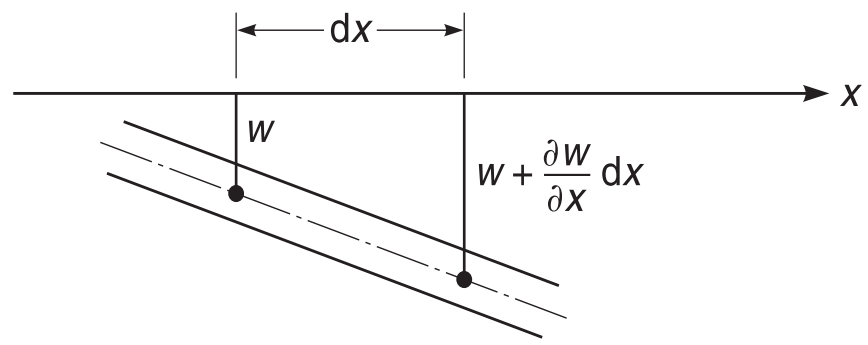

Figure 2.2.: For the Euler-Bernoulli kinematics, any finite lateral displacement induces a membrane stretching, thus making the problem non-linear [58].

to strains. In order to achieve this, we define the stiffnesses [44]

$$
A_{x x}=\int_{A} E d A, \quad B_{x x}=\int_{A} z E d A, \quad D_{x x}=\int_{A} z^{2} E d A,
$$


where the quantity $A_{x x}$ is the extensional stiffness, $B_{x x}$ is the coupled extensionalbending stiffness, and $D_{x x}$ is the bending stiffness. The role of these quantities is to couple the response of the material to the geometry of the deflection, i.e., to the in- and out-of-plane strains. Given these, the stresses are

$$
\begin{aligned}
& \sigma_{x x}=A_{x x}\left[\frac{d u_{0}}{d x}+\frac{1}{2}\left(\frac{d w}{d x}\right)^{2}\right]-B_{x x} \frac{d^{2} w}{d x^{2}}, \\
& m_{x x}=B_{x x}\left[\frac{d u_{0}}{d x}+\frac{1}{2}\left(\frac{d w}{d x}\right)^{2}\right]-D_{x x} \frac{d^{2} w}{d x^{2}},
\end{aligned}
$$

where $\sigma_{x x}$ is the membrane stress and $m_{x x}$ is the bending moment.

As seen in figure 2.2, this model hides the relationship between bending and stretching modes in the stiffness $B_{x x}$. Our aim is to instead develop a more general rod theory, which has a clear separation of membrane and bending modes. This will essentially be achieved by an intrinsic geometric kinematic description of the deformation, rather than with displacements, as done in (2.9) - (2.10).

\subsection{Rods}

We define a rod to be a 1-dimensional structural element, not necessarily straight, which is deformed to assume any planar configuration. This is a considerable simplification with respect to general rod theory, allowing for any configuration in $\mathbb{R}^{3}$, which we make in order to exclude torsional deformations from our treatment, since twist does not generalize to any 2-dimensional structure, which are the focus of this work.

\subsubsection{Euler elastica}

Leonhard Euler and Jakob Bernoulli developed the elastica theory around 1744 [32]. The elastica is the curve minimizing the following energy

$$
E[\kappa(s)]=\int_{0}^{L} \kappa(s)^{2} d s
$$

where $s$ is the arclength of the curve, $\kappa(s)$ is its curvature, and $L$ is its length. In order to find the shape of the curve, Euler moved to Cartesian coordinates and wrote equation (2.28) in terms of the Cartesian coordinates $y=f(x)$ as

$$
E=\int_{a}^{b}\left(\frac{f^{\prime \prime}}{\left(1+f^{\prime 2}\right)^{5 / 4}}\right)^{2} d x
$$

where $f^{\prime}=\partial f / \partial x$. It follows that the minimizers are solutions of the following ODE

$$
f^{\prime}=\frac{a^{2}-c^{2}+x^{2}}{\sqrt{\left(c^{2}-x^{2}\right)\left(2 a^{2}-c^{2}+x^{2}\right)}}
$$




\section{Monodimensional warm-up}

where $a$ and $c$ are parameters, which define $\lambda=a^{2} / 2 c^{2}$, the Lagrange multiplier corresponding to the implicit length constraint of (2.28). Euler performed a buckling analysis of the ODE (2.30) for $\lambda \geq 0$, which became one of the earliest examples of bifurcation theory [32].

\section{Isometric lines}

Contrary to Euler, we are interested in finding the unit-speed minimizer of (2.28), a requirement which can be explicitly added as

$$
E=\int_{a}^{b}\left(\left(\frac{f^{\prime \prime}}{\left(1+f^{\prime 2}\right)^{3 / 2}}\right)^{2}+\frac{1}{t^{2}}\left(\left(\sqrt{1+\left(f^{\prime}\right)^{2}}\right)^{2}-1\right)^{2}\right) d x .
$$

For $t \rightarrow \infty$, the Euler's elastica curve with non-constant arc-lenght is obtained. In order to obtain a truly isometric deformation, it must hold $\left\|f^{\prime}\right\|^{2}=0$. However, from Euler's ODE (2.30) it follows

$$
\left\|f^{\prime}\right\|^{2} \neq 0 \quad \text { if } \quad a, b \in \mathbb{R}
$$

This implies that for certain boundary conditions no solution exists. This counterintuitive result was determined by the unfortunate choice of parametrization. In fact, the following result holds [38].

Theorem 2.1. (Isoparametrization) Let $t \mapsto(x(t), y(t))$ be a smooth curve, then there always exists a choice for $(x(t), y(t))$ such that $x^{\prime} x^{\prime \prime}+y^{\prime} y^{\prime \prime}=0$.

With the choice $t \mapsto(x(t), y(t))$, we obtain a generalization of $(2.31)$

$$
E=\int_{a}^{b}\left(\left(\frac{x^{\prime} y^{\prime \prime}-y^{\prime} x^{\prime \prime}}{\left(\left(x^{\prime}\right)^{2}+\left(y^{\prime}\right)^{2}\right)^{3 / 2}}\right)^{2}+\frac{1}{t^{2}}\left(\left(x^{\prime}\right)^{2}+\left(y^{\prime}\right)^{2}-1\right)^{2}\right) d x
$$

for which we consider the following class of rectified curves:

$$
K_{I}=\left\{(x(t), y(t)): x^{\prime} x^{\prime \prime}+y^{\prime} y^{\prime \prime}=0\right\} .
$$

The set $K_{I}$ is merely a constraint on the parametrization: any smooth one-dimensional curve can fit into it, provided that its parametrization satisfies the above theorem.

A century after Euler's analysis, it became possible to compute closed-form rectified solutions by using Jacobi elliptic functions, with both curvature and Cartesian coordinates given as a certain function of the arclength parameter [32]. Unfortunately, the computation of elliptic integrals is not efficient and the theorem is not applicable to 2-dimensional structures, so in this work we do not look for a closed-form expression for the minimizers of (2.31), but rather for their discrete approximations. 


\subsubsection{Cosserat rod}

An alternative view is to formulate an intrinsic theory treating the rod as a set of material points forming a curve in space, which can be thought of as the line of the centroids of the cross sections of the rod [24], called the centerline. F. and E. Cosserat built their geometric theory in 1907, with the basic idea of associating intrinsic directions to each material point.

In $\mathbb{R}^{3}$, the position of a material point at $s$ is given by $r(s)=(x(s), y(s), z(s))$ with origin $r(0)=(0,0,0)$. The configuration of the rod is specified by $r(s)$ and a pair of orthonormal rod-centered unit vectors called directors $d_{1}(s)$ and $d_{2}(s)$ which span the cross section of the rod. We define

$$
d_{3}:=d_{1} \times d_{2}
$$

The tangent vector to the centerline is given by

$$
v=\frac{d r}{d s}=v_{1} d_{1}+v_{2} d_{2}+\left(v_{3}+1\right) d_{3},
$$

where $v_{1}$ and $v_{2}$ are the shear strains in the directions $d_{1}$ and $d_{2}$, respectively, and $v_{3}$ is the stretching. The strain vector is given by

$$
\epsilon=\kappa_{1} d_{1}+\kappa_{2} d_{2}+\tau d_{3},
$$

where $\kappa_{1}$ and $\kappa_{2}$ denote the curvatures and $\tau$ the twist density. The force and the moments exerted by adjacent material points are

$$
\begin{aligned}
& N(s)=N_{1} d_{1}+N_{2} d_{2}+T d_{3}, \\
& M(s)=M_{1} d_{1}+M_{2} d_{2}+M_{3} d_{3},
\end{aligned}
$$

where $N_{1}$ and $N_{2}$ are shear forces, $M_{1}$ and $M_{2}$ are bending moments, $T$ is the axial forces, and $M_{3}$ is the twisting moment about $d_{3}$. The internal energy of the rod is:

$$
\begin{aligned}
U & =\frac{1}{2}[N \cdot v+M \cdot \epsilon] \\
& =\frac{1}{2}\left[\sum_{i=1}^{2} M_{i}\left(\kappa_{i}-\tilde{\kappa}_{i}\right)+\sum_{i=1}^{2} N_{i} v_{i}+M_{3} \tau+T v_{3}\right],
\end{aligned}
$$

where $\tilde{\kappa}_{i}$ are the undeformed curvatures. In case of linear relationship between loads and strains, the internal energy becomes

$$
U=\frac{1}{2}\left[\sum_{i=1}^{2} E I_{i}\left(\kappa_{i}-\tilde{\kappa}_{i}\right)^{2}+\sum_{i=1}^{2} G A \alpha_{i} v_{i}^{2}+G J \tau^{2}+E A v_{3}^{2}\right],
$$

where $\alpha_{i}$ are shear correction factors, $E$ is the Young modulus, $G$ is the shear modulus, $A$ the cross sectional area, $I_{1,2}$ is the second moment of area about $d_{1,2}$, and $J$ is the second moment of area about $d_{3}$. The second moments of area take in account the integral over 


\section{Monodimensional warm-up}

the cross section of the strains, which are assumed to increase quadratically away from a given line. Thus, they are defined as

$$
I_{\lambda}:=\int_{A} n^{2} d A
$$

where $n$ is the distance from a given line $\lambda$ to the element $d A$.

\section{Kirchhoff rod}

A special case of the Cosserat theory is represented by the Kirchhoff rod, which has the following properties

- Inextensible $v_{3}=0$.

- Unshearable $v_{1}=v_{2}=0$.

- Isotropic $I:=I_{1}=I_{2}$.

- Initially straight $\tilde{\kappa}_{i}=0$.

The above assumptions simplify the equilibrium equations to

$$
U=\frac{1}{2}\left[E I \sum_{i=1}^{2} \kappa_{i}^{2}+G J \tau^{2}-\sum_{i=1}^{3} \lambda_{i} v_{i}\right]
$$

where $\lambda_{i}$ are Lagrange multipliers. In the case of planar rods, $\tau=v_{3}=\kappa_{2}=0$, and the above reduces to

$$
U=\frac{1}{2}\left[E I \kappa^{2}-\sum_{i=1}^{2} \lambda_{i} v_{i}\right] .
$$

As we will see throughout this work, the intrinsic view is a powerful tool in order to gain a geometric intuition for building discrete theories.

\subsection{A first encounter with locking}

We describe a discrete 1-dimensional body $\mathcal{S}_{h}$ as a graph $(V, E)$, where $V$ and $E$ are the sets of vertices and edges, respectively, with a maximum vertex degree of two. A typical choice of embedding $\phi_{h} \in X_{h}\left(\mathcal{S}_{h}\right)$ is represented by piecewise polynomials.

As asserted by Theorem 1.2, the main player for convergence is the choice of $X_{h}\left(\mathcal{S}_{h}\right)$, together with the constants $C$ and $\alpha$. In particular, the former determines the order of convergence of a discretization, while the constants are the culprit of locking.

While the accuracy of polynomial representations has been deeply investigated, locking in nonlinearly constrained systems remains a poorly understood topic in applied mathematics and computational mechanics. For these reasons, we start by presenting a well-known example of locking. We refer to [12] for more details. 


\subsubsection{Shear locking}

In the context of Finite Elements, shear locking has been observed when computations for the Timoshenko beam are performed with $w_{h}, \theta_{h} \in \mathbb{P}^{1} \cap H_{0}^{1}\left(\mathcal{S}_{h}\right)$. In the notation of chapter 1 , by defining $B(\theta, w):=w^{\prime}-\theta$, it is possible to show the lower bound

$$
\left\|w_{h}^{\prime}-\theta_{h}\right\|_{0} \geq \operatorname{ch}\left(\left\|\theta_{h}\right\|_{1}+\left\|w_{h}\right\|_{1}\right),
$$

so that from (1.8) it follows that convergence is not uniform and therefore there is locking.

Geometrically, notice first that $w_{h}^{\prime} \in \mathbb{P}^{0} \cap L^{2}\left(\mathcal{S}_{h}\right)$. Assume to look for the shear-free $\theta_{h}$ for a given configuration $w_{h}$. In general, $w_{h}^{\prime}$ is discontinuous, so there exists no $\theta_{h}$ able to fit it exactly. It follows that bending induces shearing, which means that a spurious shear stress is generated, which in turn rigidifies the system, causing locking.

\section{Remedies}

The typical cures for locking are

- Reduced integration: the term $\int\left(w_{h}^{\prime}-\theta_{h}\right)^{2} d x$ is evaluated in an averaged sense, using a 1-point quadrature at the midpoint of each edge; locking is solved since on average a continuous linear $\theta_{h}$ can approximate well a discontinuous constant $w_{h}^{\prime}$.

- Mixed methods: the shear stress $\gamma:=G\left(w^{\prime}-\theta\right) \in L^{2}$ is introduced as a Lagrange multiplier; by choosing $\gamma_{h} \in \mathbb{P}^{0} \cap L^{2}\left(\mathbb{B}_{h}\right)$ the coercivity constant $\alpha$ becomes well-conditioned, and by additionally showing the inf-sup condition [13], the discretization is proved to be convergent.

- Stabilization techniques: in the context of a mixed formulation, a triplet of elements not satisfying the inf-sup condition may still be chosen. Convergence is recovered by adding a regularization term to the energy, which vanishes as $h \rightarrow 0$, but makes possible to prove a generalized inf-sup condition [13].

- Enhanced Assumed Strains (EAS): the derivative of $w_{h}$ is enhanced by $\hat{\epsilon}_{h}$, a discontinuous linear functions vanishing at each edge midpoint; cleverly, $\hat{\epsilon}_{h}$ is chosen to exactly fill the approximation gap between $\theta_{h}$ and $w_{h}^{\prime}$.

Interestingly, in the Timoshenko case, all the above approaches turn out to be equivalent [12]. Unfortunately, this is not always the case in the case of large displacements, when the constraints generating locking are in general not linear.

\subsection{Discrete rods}

Our work is concerned with piecewise linear embeddings $X_{h}=\mathbb{P}^{1} \cap H_{0}^{1}$, which in the intrinsic language is translated into $r_{h} \in \mathbb{P}^{1} \cap H_{0}^{1}$. From this, it follows that $v_{3} \in L^{2}$ is constant over the edges and it can be computed as

$$
\left(v_{3}\right)_{h}=\sum_{i \in E} \frac{\left\|e_{i}\right\|^{2}-\left\|\bar{e}_{i}\right\|^{2}}{\left\|\bar{e}_{i}\right\|^{2}}
$$




\section{Monodimensional warm-up}

where $\bar{e}_{i}$ denotes the undeformed edge $e_{i}$. On the contrary, the curvature $\kappa \in H^{-1}$ cannot be defined pointwise, thus is assumed to be constant along the dual edges, connecting two successive edge midpoints. It can be shown that [10], in an averaged sense, it behaves as the vertex-based turning angle $\phi_{i}$

$$
\begin{aligned}
\kappa_{h} & =\sum_{i \in V} \frac{1}{\left\|\bar{e}_{i-1}\right\|+\left\|\bar{e}_{i}\right\|}\left(2 \tan \frac{\phi_{i}}{2}\right) \\
& =\sum_{i \in V} \frac{1}{\left\|\bar{e}_{i-1}\right\|+\left\|\bar{e}_{i}\right\|}\left(\frac{2 e_{i-1} \times e_{i}}{\left\|\bar{e}_{i-1}\right\|\left\|\bar{e}_{i}\right\|+e_{i-1} \cdot e_{i}}\right) .
\end{aligned}
$$

In other words, the above formula computes the curvature as a geometrically-inspired jump of the edge normal $n \in \mathbb{P}^{0} \cap L^{2}$ across vertices. This definition has showed an excellent convergence behavior when tested on various 1-dimensional benchmarks [10].

It is interesting to notice that in the Timoshenko beam, locking appears since for a given curve, a shear-free $\theta \in \mathbb{P}^{1} \cap H^{1}$ might not exist. On the contrary, in the above discrete Kirchhoff rod, given the curve, the edge normals are given uniquely. We remark that this fact alone is not a guarantee that locking is absent, as we will see in the case of shells, where normals a given surface must not pin them down uniquely, since there is no natural unique definition of discrete normals.

\subsubsection{Inextensibility and bending}

In order to offer a glimpse over the complex asymptotic behaviors that thin structures can exhibit for different loads and boundary conditions, we now examine the compatibility of the inextensibility constraint $v_{3}=0$ with a transverse load. While it might appear that in the example presented here the bending contribution is negligible, we show that it is instead fundamental for any membrane stiffness. We will perform a proper asymptotic analysis for shells in the next chapter.

Our setup is based on a straight segment with fixed vertices, loaded at its midpoint. We base our analysis on the number of iterations required by the Augmented Lagrangian (AL) algorithm [36] to enforce the inextensibility constraint $v_{3}=0$ as a function of the bending stiffness $k_{B}$. We denote with $L(x, \lambda)$ the Lagrangian of our system, where $x$ and $\lambda$ are the state variables and the Lagrange multipliers, respectively.

Numerically, it has been observed that the bending stiffness has a twofold action:

1. It accelerates each iteration. To see this, let $\lambda^{*}$ be the optimal Lagrange multipliers and recall that Newton converges quadratically if there is a strict local minimizer. This is the case if the augmented hessian

$$
\nabla^{2} L_{A}\left(x, \lambda^{*}\right)=\nabla^{2} L\left(x, \lambda^{*}\right)+\frac{1}{\mu} \nabla c(x)^{T} \nabla c(x),
$$

where $\mu \in \mathbb{R}^{+}$, is positive definite. If $\nabla^{2} L\left(x, \lambda^{*}\right)>0$ on the kernel of $\nabla c(x)$, this holds for a choice $\mu \leq \bar{\mu}$ [36]. However, there are the following problems:

- The system becomes ill-conditioned for very small $\mu$. 
- $\nabla^{2} L_{A}\left(x, \lambda^{*}\right)$ is singular if $\nabla c(x)$ is not full rank or if $\nabla^{2} L\left(x, \lambda^{*}\right)=0$, i.e., if bending is neglected.

2. It decreases the total number of iterations. To see this, consider the reasons which may cause AL to require many steps:

- Many local minima. Highly irregular solutions containing buckling are computed by the single iterations, which are far away from the final configuration.

- Very large Lagrange multipliers. This can be caused by the constraint forces acting only in-plane, i.e., nearly orthogonally to the transverse non-isometric deflections, so multipliers have to blow up to yield an exactly flat surface.

Bending helps smoothing irregular solutions and can generate forces orthogonally to the surface, so it significantly reduces the total number of iterations.
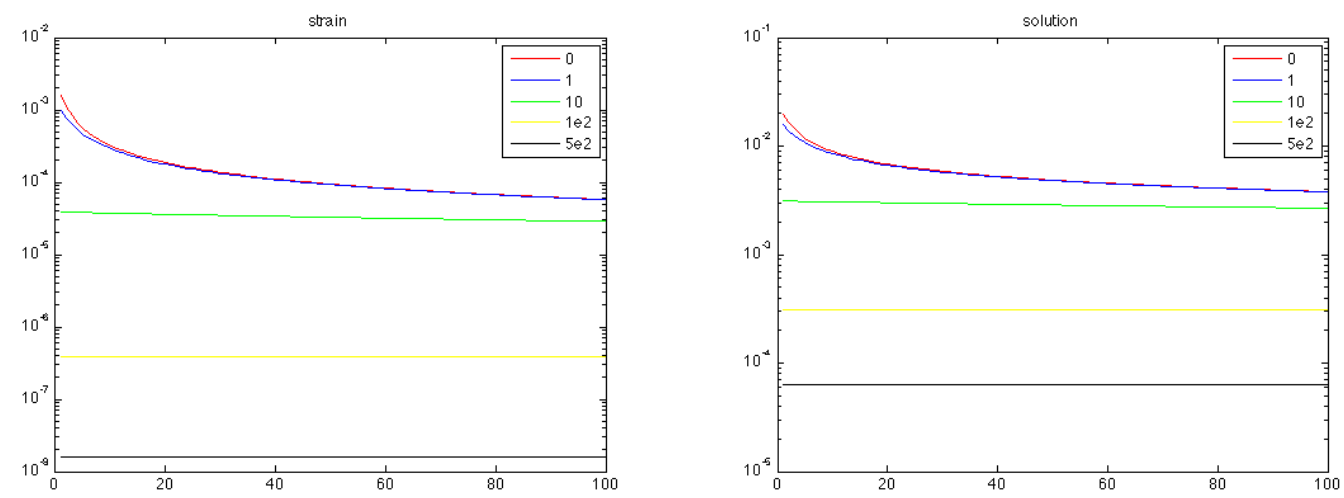

Figure 2.3.: $L^{2}$ norm of the strain and $L^{\infty}$ norm of the solution versus the iteration number of AL for several bending stiffnesses.

In figure 2.3 we compare the $L^{2}$ norm of the strain and the $L^{\infty}$ norm of the solution for several bending stiffnesses versus the number of iterations of AL for the update rule $\mu_{k}=\mu_{0}=10^{3}$. From this figure, we see that rate of convergence of AL decreases as $k_{B}$ decreases.

In figure 2.4, we consider the case $k_{B}=0$ and compare the updates $\mu_{k}=(1.2)^{k}$ and $\mu_{k}=\mu_{0}=10^{3}$. As expected, convergence is faster with the former update, however it yields a final value $\mu_{100}>10^{8}$, which leads to ill-conditioned systems. Moreover, as seen in the previous plots, the final membrane strain is larger than the one obtained with a small $\mu$ for $k_{B} \neq 0$.

\subsection{Adaptivity}

As we have seen, locking is caused by the blow-up of constants in Cea's Lemma 1.2. Therefore, given a fixed number of edges $|E|$ and vertices $|V|$, we here look for a strategy 

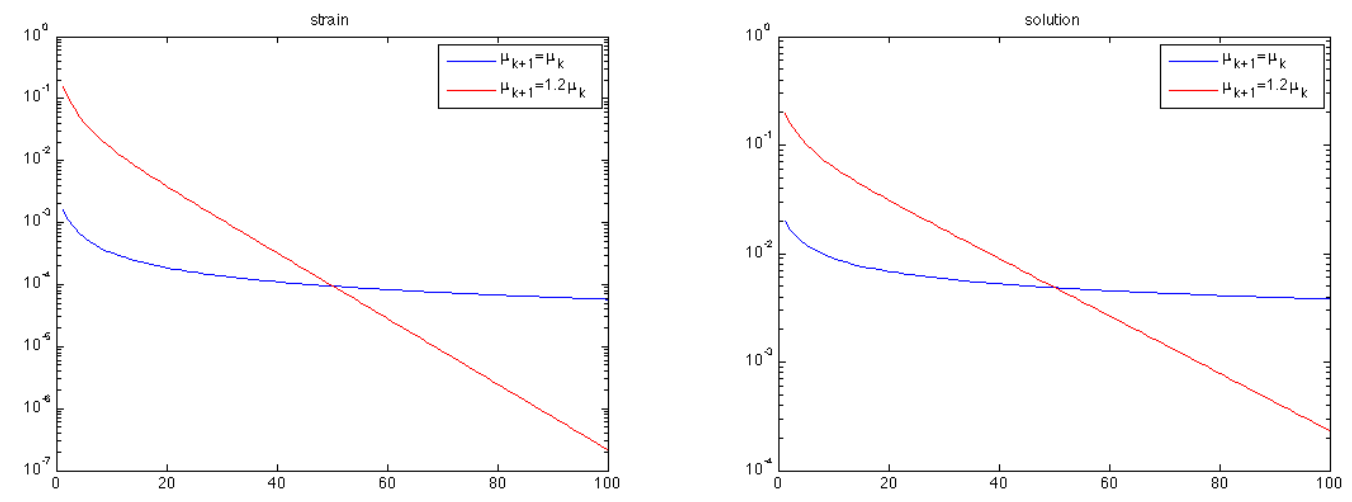

Figure 2.4.: Comparison between the updates $\mu_{k}=(1.2)^{k}$ and $\mu_{k}=\mu_{0}=10^{3}$ for $k_{B}=0$.

to compute the best possible discrete approximation of a smooth solution, such that an error estimate alike (1.5) is optimal. A solution is to look for the best placement of the nodes in the reference embedding of $\mathcal{S}_{h}$. If the optimality criterion is based on the minimization of the total energy, then we obtain the so-called $r$-adaptive method [53].

We will come back to the formalization of r-adaptivity in the following chapters. While the advantages of such strategy are clear, we here want to show the possible complications associated with it.

\subsubsection{Configurational artifacts}

Let a rod of length $L$ and distance $D$ between its endpoints be parametrized as $(\bar{x}(s), \bar{y}(s)) \in$ $\mathbb{R}^{2}$, where $s=[0,1]$. Assume the following:

- The undeformed configuration $(\bar{x}(s), \bar{y}(s))$ is curved.

- The endpoints $(x(0), y(0))=(\bar{x}(0), 0)$ and $(x(1), y(1))=(\bar{x}(1), 0)$ are fixed.

- The left vertex is clamped $\left(x^{\prime}(0), y^{\prime}(0)\right)=\left(\bar{x}^{\prime}(0), \bar{y}^{\prime}(0)\right)$.

- A point load $F(s=1)=(0,1)$ is applied.

- Segments of zero length are removed by collapsing two nodes.

Observe in Fig. 2.5 that, by moving the nodes in the undeformed positions and collapsing the overlapping ones, it is possible to obtain a straight rod of length $D \leq L$. Since the applied force $F$ is orthogonal to the straight line connecting its endpoints, such undeformed configuration is energetically the most favorable one, enhancing the flexibility of the system. However, it is a bad approximation of the smooth dotted curve.

This counterexample implies that r-adaptivity can only be used when the initial configuration has no curvature, unless an additional term measuring the distance from the smooth undeformed configuration is added to the system. 


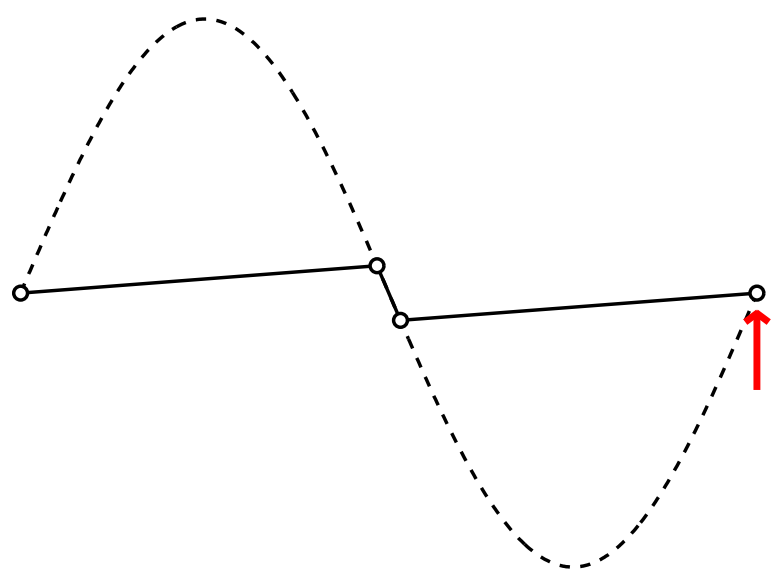

Figure 2.5.: Energy-based discretization for an applied point load (in red). The dotted line is the smooth manifold, the solid line is its approximation.

\subsubsection{Averaging artifacts}

We now consider an r-adaptive strategy based on subdividing each edge by inserting a movable point, or edgepoint, which is allowed to slide in the undeformed configuration between the two fixed adjacent vertices. As we have seen for the Discrete Kirchhoff rods, the bending energy is computed measuring the angle $\phi$ between the piecewise constant edge normals. To avoid unstable computations for degenerate segments, we compute the edge normal by averaging the two half-edge normals.

Let a rod be formed by two segments $\|A B\|=\|B C\|=L$, where $\mathrm{A}$ and $\mathrm{C}$ are fixed in space at a distance $D<2 L$. We want to compare the bending energy of the two configurations in figure 2.6.

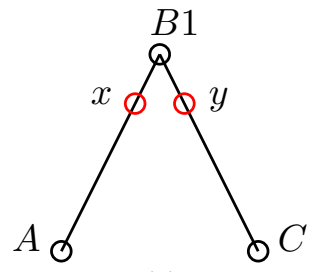

(i)

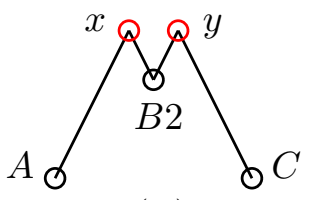

(ii)

Figure 2.6.: Two possible configurations of an adaptive rod. Edgepoints are in red.

Note that the edgepoints $\mathrm{x}$ and $\mathrm{y}$ do not sit at the material midpoint. For a bending energy of the form $E b=\sum_{i} \phi_{i}^{2}$, we obtain

$$
\begin{aligned}
(i) E b & =\phi_{B 1}^{2}, \\
(i i) E b & =\phi_{x}^{2}+\phi_{B 2}^{2}+\phi_{y}^{2} .
\end{aligned}
$$




\section{Monodimensional warm-up}

Now, assume that $\mathrm{x}$ and $\mathrm{y}$ in (ii) lie at positions $3 / 4$ and $1 / 4$, then the averaged normals to $\overline{x B 2}$ and $\overline{y B 2}$ point upwards, and we have $\phi_{B 2}=0$. We want to show that

$$
\phi_{x}^{2}+\phi_{y}^{2}<\phi_{B 1}^{2} .
$$

To see this, note that the normals to $\overline{A x}$ and $\overline{C y}$ are the same in both configurations. Therefore $\phi_{x}=\phi_{y}=\left(\phi_{B 1}\right) / 2$, and we have

$$
\phi_{x}^{2}+\phi_{y}^{2}=2\left(\frac{\phi_{B 1}}{2}\right)^{2}<\phi_{B 1}^{2}
$$

i.e. (ii) has a lower energy than (i). The above discussion gives only a brief overview of the many issues encountered in r-adaptivity, which will be further explored in chapter 5. It should be already foreseeable, though, that despite the charm of having a method for the automatic optimization of Cea's constants, the price in terms of numerical issues is ultimately not worth paying.

\subsection{Wrinkling}

From equation (2.46), we can observe that the kinematics of a discrete Kirchhoff rods is the product of considering each edge as a rigid bars, i.e., any deformation is dominated by membrane strains. Physically, we would rather like each one of them to be a simpler inextensible rod, i.e., compressions should only induce curvature. In the discrete setting, this can be achieved by enhancing the standard Cauchy-Green tensor

$$
C=\left\|v_{3}\right\|^{2}-1,
$$

with

$$
C_{\alpha}=\left\|v_{3}\right\|^{2}-1+\alpha^{2},
$$

where $\alpha \in \mathbb{R}$. The membrane energy becomes

$$
E_{m}=\inf _{\alpha \in \mathbb{R}}\left\|C_{\alpha}\right\|^{2} .
$$

Since, in the discrete setting, $v_{3}$ is approximated using (2.46), for each segment there are two cases:

- If $\left\|e_{i}\right\|>\left\|\bar{e}_{i}\right\|$, the segment is in tension, $\alpha=0$ and $C_{0}=C$.

- If $\left\|e_{i}\right\|<\left\|\bar{e}_{i}\right\|$, the segment is in compression, $\alpha^{2}=1-\left\|v_{3}\right\|^{2}$ and $C_{\alpha}=0$.

In other words, the membrane term resists to extension, but not to buckling. It follows, as illustrated in Fig. 2.7, that the discrete kinematics turns into a 1-parameter family of solutions even for a single hinge with fixed endpoints.

While this new family may be enough to solve locking, a model quantifying the curvature induced by the compression is needed, otherwise zero-energy modes would be generated. 

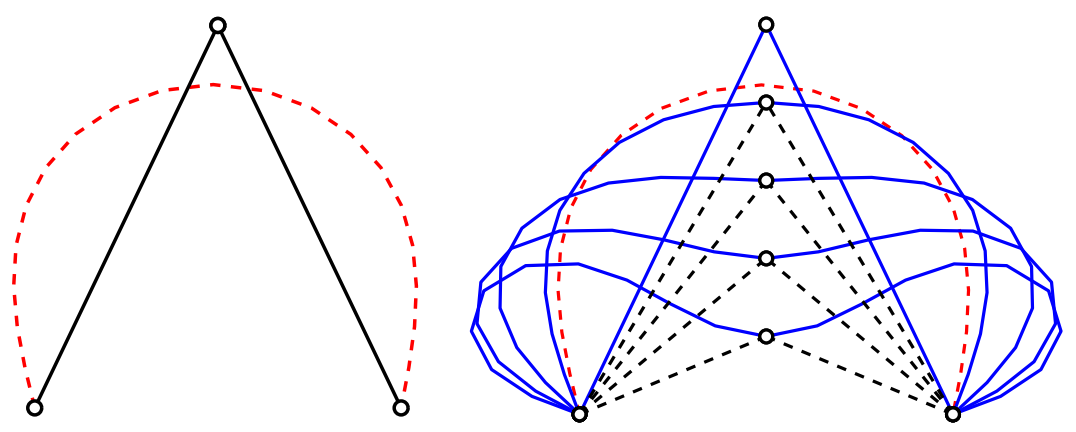

Figure 2.7.: Comparison of standard (left) and wrinkling (right) kinematics for fixed endpoints. The black lines denote the mesh, the red lines denote the elastica solution, and the blue lines denote admissible wrinkled states.

\subsubsection{Curve reconstruction}

While wrinkling models are known to be very effective at approximating membrane stresses, the implicit representation of the blue surface in Fig. 2.7 is dissatisfactory. For instance, the explicit knowledge of the blue lines is needed, e.g., for rendering or for fluid-structure interactions in graphical and engineering applications. To our knowledge, such question has never been explored so far.

More importantly, not all the members of the wrinkling family have the same bending energy but, unfortunately, the bending model proposed in equation (2.47) is unable to approximate the curvature of the blue curve from the dotted segments connecting the vertices, which would lead to the wrong discrete minimizers. The geometric reason is that the edge normals cannot be inferred from the vertex positions, but their computation must rely also on $\alpha$. In particular, two routes can be followed:

- A cubic Hermite interpolation $r_{h} \in \mathbb{P}^{3}$, based on prescribing vertex positions and normals, is computed. In this case, strain tensor is not computed pointwise, but in an integrated sense

$$
C_{\alpha}=\left(\int_{0}^{\bar{L}} v_{3} d s\right)^{2}-1,
$$

where $\bar{L}=\|\bar{e}\|$. Unfortunately, unbounded stretching is allowed by the above definition, so it is necessary to split the DOFs into in-plane and out-of-plane displacements, respectively interpolated with $u \in \mathbb{P}^{1}$ and $w \in \mathbb{P}^{3}$, with $w=0$ at the vertices. Then, the strain becomes

$$
C_{\alpha}=\left(\bar{L} \frac{\partial u}{\partial s}\right)^{2}-1+\left(\int_{0}^{\bar{L}} \frac{\partial w}{\partial s} d s\right)^{2}
$$

where we see that the last integral plays the role of $\alpha$ in (2.50). Therefore, wrinkling 
induces a modification of the vertex normals from which the bending contribution is automatically obtained.

- If we want to retain a discrete intrinsic view, at the price of lesser interpolation accuracy, an alternative is to insert a scalar DOF $e$ in each edge so that the midpoint position is computed as

$$
m_{i}=\frac{v_{i}+v_{i+1}}{2}+e n,
$$

where $n$ in the edge normal of the dotted segment connecting the vertices in figure 2.7. It follows that the constraint can be expressed as

$$
\left(\left\|m_{i}-v_{i}\right\|+\left\|m_{i}-v_{i+1}\right\|\right)^{2}-\bar{L}^{2}=0,
$$

which implicitly defines $\alpha$. The bending contribution can then be computed from equation (2.47) applied on each sub-edge. In the 1-dimensional case, such a discrete wrinkling model is exactly equivalent to a single step of h-adaptivity, with a smaller number of DOFs.

We will see in chapter 6 that in the 2-dimensional case, the above ideas are applied along the edges of the mesh, but it will be more complicated to extend such a treatment to the interior of its faces. 


\section{Smooth surfaces}

In this chapter, we consider a two-dimensional differentiable manifold $\mathcal{S}$, embedded in $\Re^{3}$. In the engineering literature, the deformation of surfaces is studied by defining the following extensions of the $1 \mathrm{D}$ theory:

- Curved planar rod theory is extended to shell theory.

- Beam theory is extended to plate theory.

The fundamental attribute of shells is the property that in-plane and out-of-plane motion are coupled due to the curvature of the undeformed configuration. Thanks to this additional rigidity, shells have become ubiquitous in engineering but, despite having received widespread attention during the whole second half of the 20th century, to date there is no general purpose discrete model suitable to all possible deformation regimes they can exhibit. In order to build such a discrete theory, we study the following regimes:

- Stretching of shells, yielding membrane theories based on 2D elasticity.

- Isometric bending of plates, known as developable surfaces in differential geometry.

This categorization augments the one used in the engineering, emphasizing the observation that developable surfaces are the geometrically nonlinear extension of plates, which are historically used to study pure bending deformations. Interestingly, while the first-order flexibility of plates subjected to an orthogonal deflection, is a trivial feature to preserve in the discrete world, in the case of developable surfaces it becomes particularly challenging, thus they are a natural setting to study membrane locking.

\subsection{Geometry of shells}

In the literature, shells are usually presented in curvilinear coordinates [40]. Instead, we prefer an equivalent intrinsic formulation, centered around the concept of a differential vector field $\mathbf{n}: \mathcal{S} \rightarrow \mathbb{R}^{3}$, for which two cases are possible:

- $\mathbf{n}$ is the unit normal field of the midsurface, which is completely determined by the embedding (Kirchhoff-Love plates and Koiter shells).

- $\mathbf{n}$ is an arbitrary unit-length vector field, thus allowing for shear (Naghdi shells and Reissner-Mindlin plates).

The generalization to a non-planar stress state, where $\mathbf{n}$ is not constrained to unit length, requires the use of a more involved constitutive model. Since our ultimate goal is to study membrane locking, we concentrate on Koiter shells. 


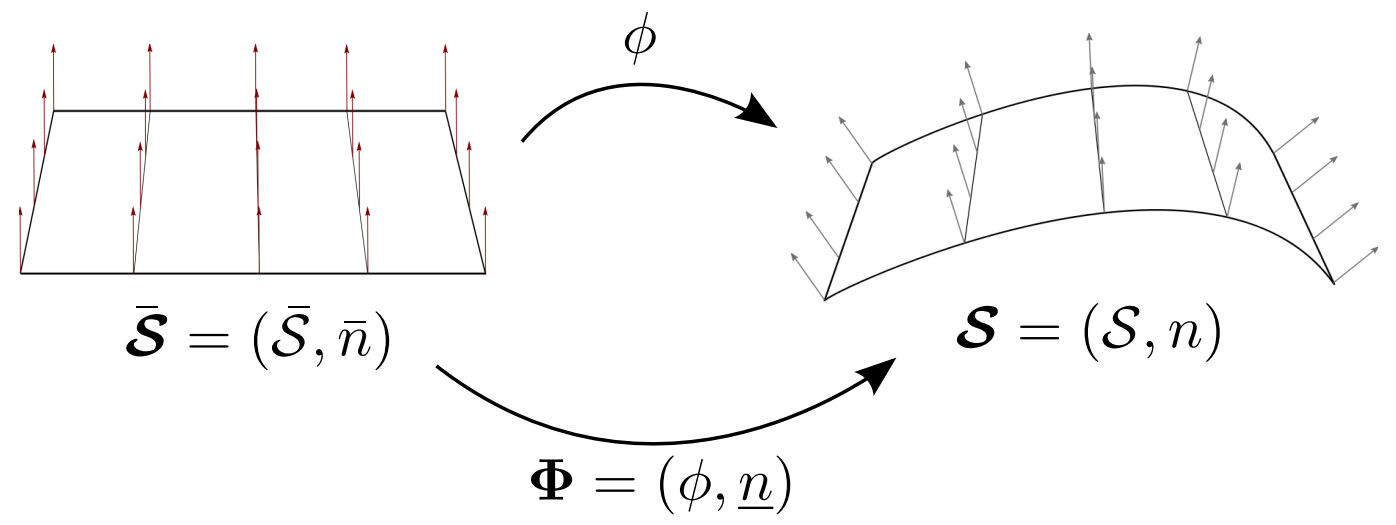

Figure 3.1.: Kinematics of a smooth shell.

\section{Kinematics}

We call $\mathbf{S} \subset \mathbb{R}^{3}$ the configuration of a shell if it can be described by an embedded twodimensional differentiable manifold $\mathcal{S}$, its mid-surface, and a thickness $t \in \mathbb{R}$. That is, any point $\mathbf{x} \in \mathbf{S}$ has associated coordinates

$$
\begin{aligned}
(x, \xi) \mathbf{S} & \in \mathcal{S} \times[-t / 2, t / 2], \\
\mathbf{x} & =x+\xi \mathbf{n}(x) .
\end{aligned}
$$

Let $\overline{\mathbf{S}}=(\overline{\mathcal{S}}, \overline{\mathbf{n}}, t)$ be the initial configuration of a shell, where $\overline{\mathbf{n}}$ is the unit normal field of the midsurface $\overline{\mathcal{S}}$. We call a deformation of $\overline{\mathbf{S}}$ a map

$$
\begin{aligned}
\mathbf{\Phi}=(\phi, \underline{\mathbf{n}}): \overline{\mathcal{S}} \times\left[-\frac{t}{2}, \frac{t}{2}\right] & \rightarrow \mathbb{R}^{3} \\
(x, \xi)_{\overline{\mathbf{S}}} & \mapsto \phi(x)+\xi \cdot \underline{\mathbf{n}}(x),
\end{aligned}
$$

such that $\phi$ is a diffeomorphism on $\overline{\mathcal{S}}$ and $\underline{\mathbf{n}}: \overline{\mathcal{S}} \rightarrow \mathbb{R}^{3}$ is a differentiable unit vector field. Then the deformed configuration is

$$
\mathbf{S}=\left(\mathcal{S}:=\phi(\overline{\mathcal{S}}), \mathbf{n}:=\underline{\mathbf{n}} \circ \phi^{-1}, t\right) .
$$

Thus $\underline{\mathbf{n}}$ us the pullback of $\mathbf{n}$ to the undeformed shell. In a similar way, we consider the undeformed 3D shell $\overline{\mathbf{S}} \subset \mathbb{R}^{3}$ as the image of :

$$
\begin{aligned}
\mathbf{X}: \overline{\mathcal{S}} \times\left[-\frac{t}{2}, \frac{t}{2}\right] & \rightarrow \overline{\mathbf{S}} \\
(x, \xi) & \mapsto x+\xi \overline{\mathbf{n}}(x) .
\end{aligned}
$$

\section{Strain}

We can describe the 3D metric tensors of the deformed and undeformed configurations through the fundamental forms of the respective midsurfaces. We recall the usual fun- 
damental forms of $\mathcal{S}$

$$
\begin{aligned}
\mathrm{I}(X, Y) & =\langle X, Y\rangle, \\
\mathrm{II}_{\mathbf{n}}(X, Y) & =\langle\operatorname{dn}(X), Y\rangle,
\end{aligned}
$$

where d denotes the (metric-free) Cartan outer derivative. If $\mathbf{S}$ is obtained by a deformation $(\phi, \underline{\mathbf{n}})$ such that $\mathcal{S}=\phi(\overline{\mathcal{S}})$ and $\mathbf{n}=\underline{\mathbf{n}} \circ \phi^{-1}$, we can write the pullbacks of these forms to the undeformed surface:

$$
\begin{aligned}
\phi^{*} \mathrm{I} & =\mathrm{d} \phi^{T} \mathrm{~d} \phi, \\
\phi^{*} \mathrm{II}_{\mathbf{n}} & =\mathrm{d} \underline{\mathbf{n}}^{T} \mathrm{~d} \phi,
\end{aligned}
$$

such that all the forms are defined on the reference configuration. In the following, we will only consider these pullbacks and therefore abuse notation and omit the pullback operator $\phi^{*}$. As the differential of $\boldsymbol{\Phi}$ writes

$$
\mathrm{d} \mathbf{\Phi}=\mathrm{d} \phi+\xi \mathrm{d} \mathbf{n}+\mathrm{d} \xi \mathbf{n},
$$

we obtain the metric tensor of the deformed configuration pulled back to $\overline{\mathcal{S}} \times[-t / 2, t / 2]$

$$
\mathbf{C}:=\mathrm{d} \boldsymbol{\Phi}^{T} \mathrm{~d} \boldsymbol{\Phi}=\mathrm{I}+2 \xi \mathrm{II}_{\mathbf{n}}+o\left(\xi^{2}\right),
$$

where we used that $\mathrm{d} \phi^{T} \mathbf{n}=0$. Similarly, for the metric tensor $\overline{\mathbf{C}}$ of the undeformed shell, pulled back to $\overline{\mathcal{S}} \times[-t / 2, t / 2]$, we get

$$
\overline{\mathbf{C}}=\mathrm{d} \mathbf{X}^{T} \mathrm{~d} \mathbf{X}=\overline{\mathrm{I}}+2 \xi \overline{\mathrm{I}}+o\left(\xi^{2}\right) .
$$

For more details, see [8].

\section{Energy}

The elastic energy of the deformation $\Phi$ is measured by the Green-Lagrange strain tensor $\mathbf{E}:=\frac{1}{2}(\mathbf{C}-\overline{\mathbf{C}})$, or more precisely, for linear material behaviour, by its weighted Frobenius norm integrated over $\overline{\mathcal{S}} \times[-t / 2, t / 2]$.

Integrating over the thickness, we obtain the energy. Following [49], with respect to smallness assumptions and constitutive relations, the shell energy for isotropic materials can be written in a concise invariant form:

$$
W=\frac{1}{2} \int_{\overline{\mathcal{S}}}\left(\frac{t}{4}\|\mathrm{I}-\overline{\mathrm{I}}\|_{M}^{2}+\frac{t^{3}}{12}\left\|\mathrm{II}_{\mathbf{n}}-\overline{\mathrm{II}}\right\|_{M}^{2}\right) \mathrm{d} \bar{A} .
$$

The norm $\|\cdot\|_{M}$ is the weighted Frobenius norm, containing the physical properties

$$
\|\overline{\mathrm{I}}\|_{M}^{2}=\frac{E}{1-\nu^{2}}\left(\nu \operatorname{tr}\left(\overline{\mathrm{I}}^{2}\right)+\frac{1}{2}(1-\nu) \operatorname{tr}(\overline{\mathrm{I}})^{2}\right),
$$

deduced by an asymptotic expansion of the 3D St. Venant-Kirchhoff model. 


\section{Smooth surfaces}

\section{Boundary conditions}

The boundary $\partial \mathcal{S}$ is assumed to be divided as follows:

- $\Gamma_{F}$ is free, where nothing is prescribed.

- $\Gamma_{S S}$ is simply supported, where $\mathbf{x}$ is prescribed.

- $\Gamma_{C}$ is clamped, where $\mathbf{x}$ and $\mathbf{n}$ are prescribed.

Given these definitions, we note immediately that the enforcement of boundary conditions depends on the choice of degrees of freedom (DOFs) for the problem. If $\mathbf{x}$ and $\mathbf{n}$ are independent variables, then the above conditions are easily used in the framework of variational calculus to derive the equilibrium equations from the elastic energy. If a different set is chosen, then the conditions could become much more complicated, as we will see in the following.

To study the curvature of shells, the typical approach is to consider plates, defined by the assumption $\overline{\mathrm{II}}=0$, in order to express in coordinates the membrane and bending deformations of a shell. Additionally, if small deformations are assumed, the two energy contributions become uncoupled. We present these theories in the next section.

\subsection{Plates}

In continuum mechanics, plate theories are mathematical descriptions of the mechanics of flat plates that draw on the theory of beams [54]. Of the numerous plate theories that have been developed since the late 19th century, two are widely accepted and used in engineering. These are the Kirchhoff-Love and the Reissner-Mindlin plate theories, which are linked to the Euler-Bernoulli and Timoshenko beam theories, respectively. From a mathematical standpoint, however, the most important theory is the Föppl-von Karman plate, thanks to its geometric interpretation, which we discuss below.

\subsubsection{Kinematic assumptions}

Let the undeformed position and the displacement of a point be denoted with $\mathbf{x}$ and $\mathbf{u}(\mathbf{x})$, respectively. In coordinates, they are expressed as

$$
\mathbf{x}=\sum_{i=1}^{3} x_{i} \boldsymbol{e}_{i}, \quad \mathbf{u}=\sum_{i=1}^{3} u_{i} \boldsymbol{e}_{i}
$$

where the vectors $\boldsymbol{e}_{i}$ form a Cartesian basis with origin on the mid-surface of the plate, $x_{1}$ and $x_{2}$ are the Cartesian coordinates on the mid-surface of the undeformed plate, and $x_{3}$ is the coordinate for the thickness direction.

The displacement can be decomposed into a vector sum of the mid-surface displacement and an out-of-plane displacement $w_{0}$ in the $x_{3}$ direction. We can write the in-plane 
displacement of the mid-surface as

$$
\mathbf{u}^{0}=\sum_{\alpha=1}^{2} u_{\alpha}^{0} \boldsymbol{e}_{\alpha}
$$

\section{Kirchhoff-Love assumptions}

The Kirchhoff-Love theory of plates is a two-dimensional mathematical model that is used to determine the stresses and deformations in thin plates subjected to forces and moments. This theory is an extension of Euler-Bernoulli beam theory and was developed in 1888 by Love [33], using assumptions proposed by Kirchhoff.
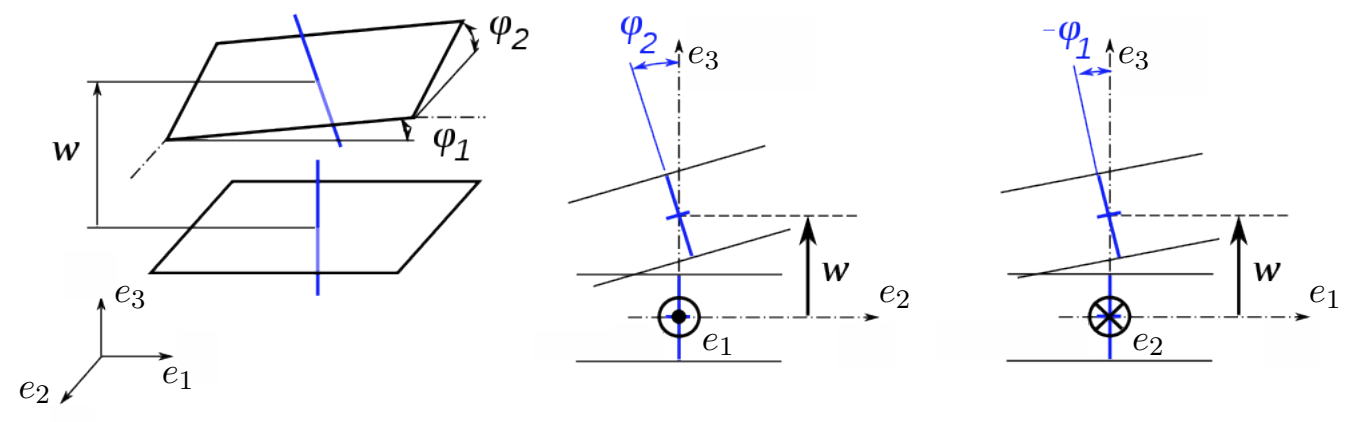

Figure 3.2.: Displacement in a thin Kirchhoff-Love plate: vertical displacement $w$ and rotations $\varphi_{1}$ and $\varphi_{2}$ of the middle plane.

In the Kirchhoff description of thin plates, it is assumed that during the deformation the following are verified: (i) straight lines normal to the mid-surface remain straight after the deformation, (ii) straight lines normal to the mid-surface remain normal to the mid-surface the after deformation, and (iii) the thickness of the plate does not change during the deformation.

Then, the Kirchhoff hypotheses imply that $u_{\alpha}$ is the first order Taylor series expansion of the displacement around the mid-surface

$$
\begin{aligned}
& u_{\alpha}(\mathbf{x})=u_{\alpha}^{0}\left(x_{1}, x_{2}\right)-x_{3} \frac{\partial w^{0}}{\partial x_{\alpha}} \equiv u_{\alpha}^{0}-x_{3} w_{, \alpha}^{0}, \quad \alpha=1,2, \\
& u_{3}(\mathbf{x})=w^{0}\left(x_{1}, x_{2}\right)
\end{aligned}
$$

If $\theta_{\alpha}$ are the angles of rotation of the normal to the mid-surface, then in the KirchhoffLove theory

$$
\theta_{\alpha}=w_{, \alpha}^{0}
$$




\section{Smooth surfaces}

\section{Reissner-Mindlin assumptions}

The Reissner-Mindlin theory of plates is an extension of Kirchhoff-Love plate theory that takes into account shear deformations through the thickness of a plate. The theory was proposed in 1951 by Raymond Mindlin. A similar, but not identical, theory had been proposed earlier by Eric Reissner in 1945. Both theories are intended for thick plates in which the normal to the mid-surface remains straight but not necessarily perpendicular to the mid-surface.

Relaxing Kirchhoff's hypothesis (ii) implies that the displacements in the ReissnerMindlin plate theory have the form

$$
\begin{aligned}
& u_{\alpha}(\mathbf{x})=u_{\alpha}^{0}\left(x_{1}, x_{2}\right)-x_{3} \theta_{\alpha}, \quad \alpha=1,2 \\
& u_{3}(\mathbf{x})=w^{0}\left(x_{1}, x_{2}\right)
\end{aligned}
$$

Unlike Kirchhoff-Love plate theory, Mindlin's theory assumes that $\theta_{1} \neq w_{, 1}^{0}$ and $\theta_{2} \neq w_{, 2}^{0}$, thereby incorporating first-order shear effects.

\subsubsection{Strains}

For the situation where the strains in the plate are infinitesimal and the rotations of the mid-surface normals are small, the 3D strain-displacement relations are

$$
\epsilon(u)=\mathrm{I}-\overline{\mathrm{I}} \approx \frac{1}{2}\left(\nabla u+\nabla u^{T}\right) .
$$

Explicitly, the components of the strain tensor are

$$
\begin{aligned}
\varepsilon_{\alpha \beta} & =\frac{1}{2}\left(\frac{\partial u_{\alpha}}{\partial x_{\beta}}+\frac{\partial u_{\beta}}{\partial x_{\alpha}}\right) \equiv \frac{1}{2}\left(u_{\alpha, \beta}+u_{\beta, \alpha}\right), \quad \alpha, \beta=1,2, \\
\varepsilon_{\alpha 3} & =\frac{1}{2}\left(\frac{\partial u_{\alpha}}{\partial x_{3}}+\frac{\partial u_{3}}{\partial x_{\alpha}}\right) \equiv \frac{1}{2}\left(u_{\alpha, 3}+u_{3, \alpha}\right), \quad \alpha=1,2, \\
\varepsilon_{33} & =\frac{\partial u_{3}}{\partial x_{3}} \equiv u_{3,3} .
\end{aligned}
$$

Using the Reissner-Mindlin kinematics, we obtain the plane-stress condition $\varepsilon_{33}=0$ and

$$
\begin{aligned}
\varepsilon_{\alpha \beta} & =\frac{1}{2}\left(u_{\alpha, \beta}^{0}+u_{\beta, \alpha}^{0}\right)-\frac{x_{3}}{2}\left(\theta_{\alpha, \beta}+\theta_{\beta, \alpha}\right), \\
\varepsilon_{\alpha 3} & =\frac{1}{2}\left(w_{, \alpha}^{0}-\theta_{\alpha}\right),
\end{aligned}
$$

where $\alpha, \beta=1,2$. The shear strain $\varepsilon_{\alpha 3}$ is assumed to be constant across the thickness of the plate. Unfortunately, this is not accurate since the shear stress is known to be parabolic even for simple plate geometries. To account for the inaccuracy in the shear strain, a correction factor $\kappa$ is applied

$$
\varepsilon_{\alpha 3}=\frac{1}{2} \kappa\left(w_{, \alpha}^{0}-\theta_{\alpha}\right) .
$$


Using the Kirchhoff-Love assumptions, we obtain

$$
\varepsilon_{\alpha 3}=-w_{, \alpha}^{0}+w_{, \alpha}^{0}=0 .
$$

Therefore, the only non-zero strains are in the in-plane directions, which is a consequence of assuming that there is a linear variation of displacement across the plate thickness but the plate thickness does not change during deformation. This implies that the normal stress through the thickness is ignored; an assumption which is also called the plane stress condition.

\subsubsection{Energy}

For an isotropic and homogeneous plate, the Hooke's stress-strain relations are

$$
\left[\begin{array}{l}
\sigma_{11} \\
\sigma_{22} \\
\sigma_{12}
\end{array}\right]=\frac{E}{1-\nu^{2}}\left[\begin{array}{ccc}
1 & \nu & 0 \\
\nu & 1 & 0 \\
0 & 0 & 1-\nu
\end{array}\right]\left[\begin{array}{l}
\varepsilon_{11} \\
\varepsilon_{22} \\
\varepsilon_{12}
\end{array}\right]
$$

where $E$ is the Young modulus and $\nu$ is the Poisson ratio, while the shear stresses and strains are related by

$$
\sigma_{3 \alpha}=2 G \varepsilon_{3 \alpha} \quad \alpha=1,2,
$$

where $G=E /(2(1+\nu))$ is the shear modulus. The corresponding energy is

$$
E=\int_{\Omega_{0}} \int_{-h}^{h} \sigma: \epsilon d x_{3} d \Omega=\int_{\Omega_{0}} \int_{-h}^{h} \sum_{\alpha, \beta=1}^{2}\left(\sigma_{\alpha \beta} \epsilon_{\alpha \beta}+2 \kappa \sigma_{3 \alpha} \epsilon_{3 \alpha}\right) d x_{3} d \Omega
$$

Interestingly, the Reissner-Mindlin kinematics can be interpreted as a penalty method to enforce the constraint $\epsilon_{\alpha 3}=0$, which is built-in in the Kirchhoff-Love kinematics. This is fortunate, since in general the explicit knowledge of an appropriate kinematics for any given constraint is not easy. In the limit $G \rightarrow \infty$, the shear stress $\sigma_{3 \alpha}$ acts as a Lagrange multiplier but, in general, $G$ is sufficiently high so that the two models produce the same results, although from a mathematical standpoint they have to be treated differently in the discrete setting.

From the principle of virtual works, it is possible to derive the equilibrium equations in terms of shear stresses and bending moments, referring to [54] for more details. In the simplified case of pure bending under a transverse load $q(x)$, the in-plane displacements are zero, therefore the resulting equilibrium equation is

$$
\frac{2 h^{3} E}{3\left(1-\nu^{2}\right)} \Delta^{2} w=q
$$

which is known as the biharmonic equation. 


\section{Smooth surfaces}

\subsubsection{Föppl-von Karman plate}

The Föppl-von Karman theory is valid for large displacements and small rotations. It is important to present this model, since it is used to study the isometric large deformations of plates [4]. The kinematics and the energy follow the Kirchhoff-Love theory, so we limit the presentation to the strain-displacement relations, which are obtained by considering the nonlinear contribution of large deflections $w$ on the in-plane strain

$$
\begin{aligned}
\varepsilon_{\alpha \beta} & =\frac{1}{2}\left(u_{\alpha, \beta}+u_{\beta, \alpha}\right)+\frac{1}{2} w_{, \alpha} w_{, \beta} \\
& =\frac{1}{2}\left(u_{\alpha, \beta}^{0}+u_{\beta, \alpha}^{0}+w_{, \alpha}^{0} w_{, \beta}^{0}\right)-x_{3} w_{, \alpha \beta}^{0}, \quad \alpha, \beta=1,2,
\end{aligned}
$$

while $\epsilon_{\alpha 3=0}$, as in the Kirchhoff-Love theory. As we have seen in Fig. 2.2, this theory is not invariant under rotations, therefore it is appropriate only for the situation where the rotations of the mid-surface normals are moderate, i.e., in the range between 10 and 15 degrees. However, this approximation is physically more consistent than the linear strain (3.19), since in this case the omitted terms are all of the same order in terms of the displacements $u$ and $w$ [4].

\subsection{Physics of shells}

Plates have been a very important tool for engineers and physicists to simplify shell theories and to carry on explicit calculations in several deformation scenarios. However, we find that they do not offer enough insights to build discrete shell theories from first principles. Instead, we first ask if the shell theory converges towards a limit model when $t \rightarrow 0$. Then, we concentrate on studying such limit models independently, with the goal of mimicking these behaviors in the discrete setting. In our presentation, we follow [14] and [40], providing more details regarding the intuition behind the asymptotic regimes. We start by writing the shell energy as

$$
\inf _{u_{t} \in W}\left(t^{3} A_{b}\left(u_{t}, u_{t}\right)+t A_{m}\left(u_{t}, u_{t}\right)-F_{t}\left(u_{t}\right)\right),
$$

where the bending and membrane energies $A_{b}$ and $A_{m}$ are independent of $t, F_{t}$ is the external virtual work, and $W$ is a Sobolev space. We assume that essential boundary conditions are prescribed in such a way that no rigid motion is allowed, so $u_{t} \in W$ solves

$$
t^{3} A_{b}\left(u_{t}, v\right)+t A_{m}\left(u_{t}, v\right)=F_{t}(v) \quad \forall v \in W .
$$

In order to study the asymptotic behavior as $t$ tends to zero, we scale the load

$$
F_{t}(u)=t^{\rho} G(u),
$$

where $G \in W^{\prime}$ must be independent of $t$ and $\rho \in \Re$. As shown in [5], there exists at most one exponent $\rho$ that provides an admissible asymptotic behavior, which is equivalent to having a finite non-zero limit for the equivalent scaled energy

$$
t^{3-\rho} A_{b}\left(u_{t}, u_{t}\right)+t^{1-\rho} A_{m}\left(u_{t}, u_{t}\right) .
$$


In such case it can be shown [5] that

$$
1 \leq \rho \leq 3 .
$$

Additionally, we introduce a closed subspace of $W$, characterized by pure-bending inextensional displacements [14]

$$
W_{0}=\left\{v \in W \mid A_{m}(v, v)=0\right\},
$$

Depending on $W_{0}$, the shell is said to have:

- non-inhibited pure bending, if $W_{0} \neq\{0\}$,

- inhibited pure bending, if $W_{0}=\{0\}$.

\subsubsection{Non-inhibited shells}

This situation is analogous to beams and rods with only one fixed end. In particular, we distinguish between two cases:

- If $\exists u_{0} \in W_{0}: G(W) \neq 0$, we say that the load activates the pure bending displacements and it can be shown that $\rho=3$ [5]. The limit problem is given by:

Find $u_{0} \in W_{0}$ such that

$$
A_{b}\left(u_{0}, v\right)=G(v), \quad \forall v \in W_{0}
$$

and the following proposition holds:

Proposition 3.1. Assume that $W_{0} \neq 0$. Then, setting $\rho=3$, $u_{t}$ converges strongly into $W$ to $u_{0}$, the solution of (3.39). Moreover, we have:

$$
\lim _{t \rightarrow 0} \frac{1}{t^{2}} A_{m}\left(u_{t}, u_{t}\right)=0
$$
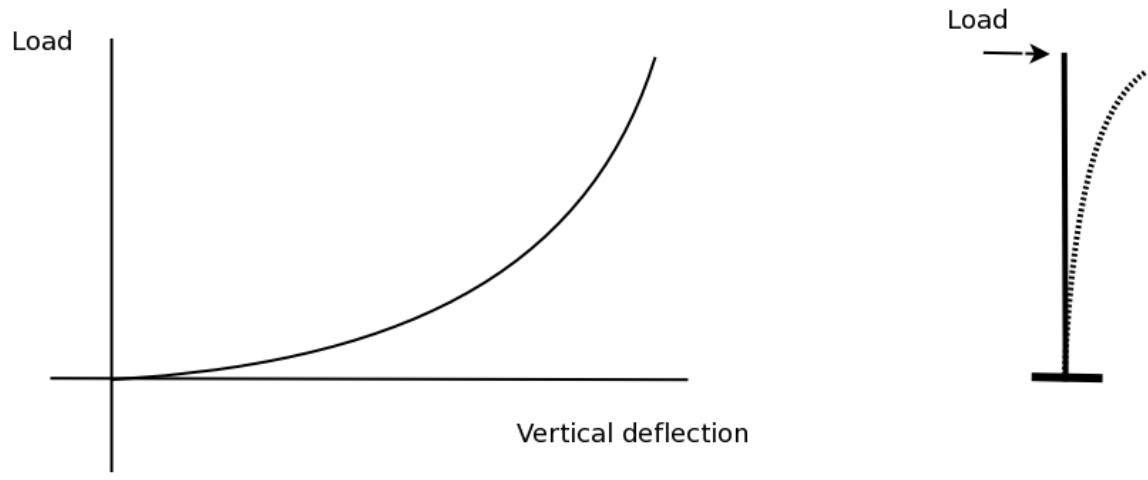

Figure 3.3.: Load vs deflection at the tip (left) for a bending-dominated deformation of a clamped beam (right). The load causes a pure bending deformation with negligible stretching. 

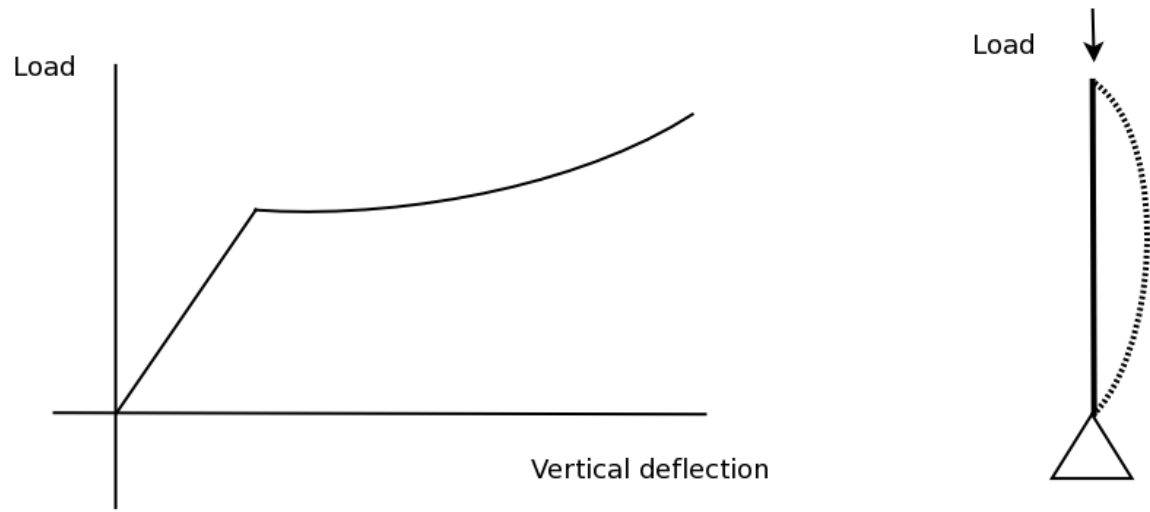

Figure 3.4.: Load vs deflection at the tip (left) for an unstable membrane-dominated deformation of a hinged beam (right). The load causes a small compression until the bucking point is reached.

- When the loading does not activate $\kappa_{0}$, we obtain an unstable membrane-dominated situation [5], as shown in figure 3.4.

\subsubsection{Inhibited shells}

In this case, $W_{0}=0$ implies that $A_{m}$ is positive definite, thus we introduce the subspace $W_{m}$, which is the completion of the space $W$ with respect to the membrane norm $\|\cdot\|_{m}$ defined by the bilinear form $A_{m}$ :

$$
\|W\|_{m}=A_{m}(v, v), \quad v \in W
$$

- If $G \in W_{m}^{\prime}$, the loading can be resisted by membrane stresses only [40] and we call $G$ admissible. The adequate load-scaling exponent corresponds to $\rho=1$ and the membrane-dominated limit problem reads: Find $u_{m} \in W_{m}$ such that

$$
A_{m}\left(u_{m}, v\right)=G(v), \quad \forall v \in W_{m} .
$$

Furthermore, the following proposition holds [5]:

Proposition 3.2. Assume that pure bending is not inhibited and also that $G \in$ $W_{m}^{\prime}$. Then, setting $\rho=1, u_{t}$ converges strongly in $W_{m}$ to $u_{m}$ the solution of 3.42. Moreover, we have:

$$
\lim _{t \rightarrow 0} t^{2} A_{b}\left(u_{t}, u_{t}\right)=0
$$



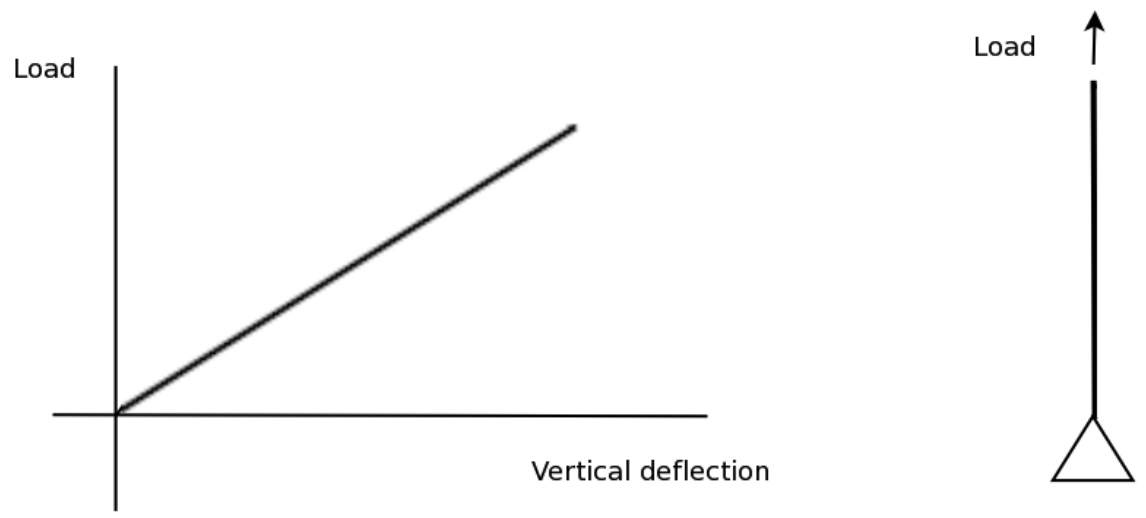

Figure 3.5.: Load vs deflection at the tip (left) for a membrane-dominated deformation of a hinged beam (right). For such a load, the bending stiffness is negligible

- If $\mathrm{G}$ is a non-admissible membrane loading, the membrane problem is ill-posed, i.e., other admissible asymptotic behaviors may exist with $1<\rho<3[5]$, as seen in figure 3.6. These regimes are called boundary layers, since they typically contain complex deformations in a very narrow part of the domain.

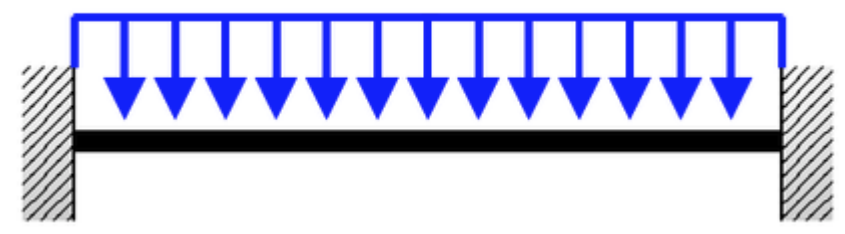

Figure 3.6.: Ill-posed membrane situation. The bending stiffness is negligible almost everywhere, except very close to the boundary points, where boundary layers are formed.

Remark. Even if the geometric nature of the midsurface plays a crucial role in the asymptotic behavior, $W_{0}$ depends on the boundary conditions that together with the equation $A_{m}(u, v)=0$ define a Cauchy problem [5]. For example, when considering elliptic surfaces, imposing zero displacements on the whole boundary is sufficient to inhibit pure bending displacements and the membrane problem set in $W_{m}$ is well-posed. But if the displacements are fixed only on a limited part of the boundary, we obtain an ill-posed membrane problem [30].

\subsection{Membranes}

We now concentrate on inhibited shells, in particular to membrane-dominated deformations, for which we can simplify the Koiter model by assuming

$$
\mathrm{II} \approx \overline{\mathrm{II}} .
$$




\section{Smooth surfaces}

Additionally, if we restrict ourselves to the case $\overline{\mathrm{II}}=0$, we recover $2 \mathrm{D}$ elasticity. In the case of small rotations, the energy becomes quadratic and the problem can be formulated by using the strain (3.19). There are several variational formulations which can be used in order to obtain a solution of the elastic problem. While all equivalent in the smooth setting, they in general lead to very different discrete approximations. In presenting them, we assume homogeneous boundary conditions and linear strain-stress relations.

\section{Displacement formulation}

The basic formulation of linear elasticity looks for a minimizer $\phi \in H^{1}$ of the energy

$$
\int_{\overline{\mathcal{S}}}(\epsilon(\phi): C \epsilon(\phi)-f \phi) \mathrm{d} \bar{A}
$$

where $C$ is the elastic stiffness tensor and $\epsilon(\phi)$ is defined as in (3.19).

Lemma 3.3. (Korn's second inequality) Let $\mathcal{S} \subset \Re^{d}$ be an open bounded set with Lipschitz boundary. Suppose that the solution vanishes on $\mathcal{S}_{0} \subset \partial \mathcal{S}$, having positive $(d-1)$-dimensional measure. Then there exists a positive number $b=b\left(\mathcal{S}, \Gamma_{0}\right)$ such that

$$
\int_{\Omega} \epsilon(v): \epsilon(v) d x \geq b\|v\|_{1}^{2} \quad \forall v \in H_{\Gamma}^{1}(\mathcal{S})
$$

Here $H_{\Gamma}^{1}$ is the closure of $\left\{v \in C^{\infty}(\mathcal{S})^{3} ; v(x)=0\right.$ for $\left.x \in \Gamma_{0}\right\}$ w.r.t. the $\|\cdot\|_{1}$-norm.

For a proof, see [12]. Korn's second inequality implies that the energy (3.45) is elliptic, from which it follows that the solution exists and it is unique. Geometrically, this result is very important since $\epsilon(v)=0$ is verified if and only if $v(x)=A x+b$, where $A$ is a skew-symmetric matrix and $b \in \Re^{d}$. Thanks to Korn's second inequality, $\epsilon(v)=0$ implies also $\nabla v=0$, from which we conclude that infinitesimal rotations must induce stretching.

\section{Hellinger-Reissner}

The Hellinger-Reissner principle says that the solution $(\phi, \sigma) \in H^{1} \times L^{2}$ is a stationary point of the action

$$
\int_{\overline{\mathcal{S}}}\left(\sigma:\left(C^{-1} \sigma-\epsilon(\phi)\right)-f \phi\right) \mathrm{d} \bar{A}
$$

where $\epsilon(\phi)$ is defined as in (3.19). This principle is probably the most successful one in the literature and we will come back to it in order to analyze the approximation of membranes using nonconforming piecewise linear elements. Since this principle is a saddle-point, it requires the fulfillment of the inf-sup condition to be well-posed [13]. Fortunately, if Korn's second inequality is satisfied, it can be shown that the inf-sup condition follows immediately [12]. 


\section{Hu-Washizu}

The Hu-Washizu principle says that the solution $(\phi, \sigma, \epsilon) \in H^{1} \times L^{2} \times L^{2}$ is a stationary point of the action

$$
\int_{\overline{\mathcal{S}}}(\epsilon:(C \epsilon-\sigma)+\sigma: \nabla \phi-f \phi) \mathrm{d} \bar{A} .
$$

We do not develop further this theory since, unfortunately, most of the known approximations using the $\mathrm{Hu}$-Washizu principle were proved to be equivalent to the HellingerReissner theory. For more details, see [12].

\subsection{Developable surfaces}

We now concentrate on inhibited shells, in particular to bending-dominated deformations, described by the set of inextensible deformations

$$
W_{0}=\{\phi: \mathrm{I}=\overline{\mathrm{I}}\},
$$

which simplifies the Koiter theory to

$$
\inf _{\phi \in W_{0}} \int_{\overline{\mathcal{S}}} \frac{t^{3}}{24}\left\|\mathrm{II} \mathrm{I}_{\mathbf{n}}-\overline{\mathrm{II}}\right\|_{M}^{2} \mathrm{~d} \bar{A}-\int_{\overline{\mathcal{S}}} f \phi \mathrm{d} \bar{A},
$$

where $f$ is an external load. Furthermore, if $\overline{\mathrm{II}}=0$, the above simplifies to Willmore's energy

$$
\inf _{\phi \in W_{0}} \frac{E}{1-\nu^{2}} \int_{\overline{\mathcal{S}}} \frac{t^{3}}{24}\left(H^{2}+2(1-\nu) K\right)-\int_{\overline{\mathcal{S}}} f \phi \mathrm{d} \bar{A},
$$

where $H$ and $K$ are the mean and the Gauss curvatures of the surface. Recalling that, under isometric deformations, $K=0$ and $H$ can be computed linearly in term of the coordinates, it follows that the energy is in fact quadratic

$$
\inf _{\phi \in W_{0}} \frac{E}{1-\nu^{2}} \int_{\overline{\mathcal{S}}} \frac{t^{3}}{24}(\Delta \mathbf{x})^{2}-\int_{\overline{\mathcal{S}}} f \phi \mathrm{d} \bar{A} .
$$

Interestingly, the above quadratic energy is equivalent to the small displacement KirchhoffLove theory up to the topological invariant Euler characteristic $\chi$

$$
\begin{aligned}
\int_{\overline{\mathcal{S}}} \frac{t^{3}}{24} \sigma: \epsilon \mathrm{d} \bar{A} & =\frac{E}{1-\nu^{2}} \int_{\overline{\mathcal{S}}} \frac{t^{3}}{24}\left(\operatorname{tr}(\epsilon)^{2}+2(1-\nu) \operatorname{det}(\epsilon)\right) \mathrm{d} \bar{A} \\
& =\frac{E}{1-\nu^{2}} \int_{\overline{\mathcal{S}}} \frac{t^{3}}{24}\left(\operatorname{tr}\left(\nabla^{2} \mathbf{x}\right)^{2}+2(1-\nu) \operatorname{det}\left(\nabla^{2} \mathbf{x}\right)\right) \mathrm{d} \bar{A} \\
& =\frac{E}{1-\nu^{2}} \int_{\overline{\mathcal{S}}} \frac{t^{3}}{24}(\Delta \mathbf{x})^{2} \mathrm{~d} \bar{A}+\frac{t^{3} E}{6(1+\nu)} \pi \chi,
\end{aligned}
$$

where we have used Gauss-Bonnet theorem [38]. However, while (3.51) is defined under the assumption of isometric deformations but for any displacement and rotation, equation (3.29) is defined under the assumption of infinitesimal displacements. This is the reason why we consider developable surfaces and not plates, to be the right mathematical object of interest to study bending-dominated deformations. 


\section{Smooth surfaces}

\section{Saddle-point formulation}

Since the knowledge of $W_{0}$ is not available in general, we can instead consider a developable surface as a constrained system, with kinematics given by the inextensible limit of the membrane term. By doing so, the problem (3.51) is written on a simpler space using Lagrange multipliers

$$
\inf _{\mathbf{x} \in H_{0}^{2}} \sup _{q \in L^{2}} \frac{1}{2} \int_{\overline{\mathcal{S}}}(\Delta \mathbf{x})^{2} d s-\frac{1}{2} \int_{\overline{\mathcal{S}}} q:\left(\nabla \mathbf{x} \nabla \mathbf{x}^{T}-I d\right) d s-\int_{\overline{\mathcal{S}}} f \mathbf{x} d s,
$$

or, in abstract form:

$$
\inf _{\mathbf{x} \in H_{0}^{2}} \sup _{q \in L^{2}} \frac{1}{2} a(\mathbf{x}, \mathbf{x})-b(q, \mathbf{x})-\langle f, \mathbf{x}\rangle
$$

which, defining the following trilinear form

$$
c(u, v, q)=\frac{1}{4} \int_{\overline{\mathcal{S}}} q\left(\nabla u^{T} \nabla v+\nabla v^{T} \nabla u\right) d s=\langle C(u, v), q\rangle,
$$

being in 1-to-1 correspondence with $b()$ and continuous since $W^{2,2} \hookrightarrow W^{1, p} \forall p \geq 2$ according to Sobolev embedding theorem, can be rewritten as

$$
\inf _{\mathbf{x} \in H_{0}^{2}} \sup _{q \in L^{2}} \frac{1}{2} a(v, v)-c(v, v, q)-\langle f, v\rangle-\langle I, q\rangle,
$$

whose Euler-Lagrange equations for the solution $(\mathbf{x}, p) \in W \times L^{2}$ are

$$
\begin{aligned}
a(\mathbf{x}, v)-2 c(\mathbf{x}, v, p) & =\langle f, v\rangle_{W} \quad \forall v \in H_{0}^{2} \\
c(\mathbf{x}, \mathbf{x}, q) & =\langle I, q\rangle_{L}^{2} \quad \forall q \in L^{2} .
\end{aligned}
$$

However, it is not straightforward to apply the theory of saddle-point problems found, e.g., in [13], since the above problem is subjected to nonlinear constraints. Therefore, in the following chapters, rather than focusing on the analysis of the abstract problem, we will concentrate on devising a discrete theory of developable surfaces.

This is usually done either by discretizing equation (3.11) with small $t$, which usually yields an ill-conditioned problem, or by approximating equation (3.53), which involves the analysis of a saddle-point problem to avoid locking. Alternatively, in Chapter 6 we will tackle the problem from first principles, i.e., by defining a discrete kinematics through suitable constraints, which can be considered the inextensible limit of a possibly unknown discrete membrane energy.

\section{Boundary conditions}

To underline the difficulties arising when $\mathbf{n}$ is not part of the unknowns, we present the derivation of appropriate boundary conditions for the constrained formulation of 
developable surfaces. The main task is to integrate by parts a term of the form $\int_{\overline{\mathcal{S}}} p$ : $a^{T} \nabla v$, which results in

$$
\int_{\partial \Omega} \sum_{i, j}\left(a_{i j} v_{i}\right)\left(p_{1 j}, p_{j 2}\right) \cdot n-\int_{\overline{\mathcal{S}}} \sum_{i, j}\left(\nabla a_{i j} v_{i}\right)^{T}\left(p_{1 j}, p_{j 2}\right)+\left(\nabla a_{i j} v_{i}\right) \nabla \cdot\left(p_{1 j}, p_{j 2}\right) .
$$

In a more compact notation, integration by parts of the trilinear form is written as

$$
c(\mathbf{x}, v, p)=\int_{\Gamma_{F}} \nabla \mathbf{x}^{T} v \cdot p n-2 \int_{\overline{\mathcal{S}}}\left(\nabla \mathbf{x}^{T} v \cdot \operatorname{div}(p)+\nabla^{2} \mathbf{x}^{T} v: p\right),
$$

where we have used that $\left.v\right|_{\Gamma_{S S} \cup \Gamma_{C}}=0$. For the bending part, we have

$$
a(\mathbf{x}, v)=\int_{\Gamma_{F}} \frac{\partial(\Delta \mathbf{x})}{\partial n} v+\int_{\Gamma_{F} \cup \Gamma_{S S}} \Delta \mathbf{x} \frac{\partial v}{\partial n}-\int_{\overline{\mathcal{S}}} \Delta^{2} x v .
$$

Therefore, the natural conditions are

$$
\begin{array}{rlll}
\Delta \mathbf{x}=0 & \text { on } & \Gamma_{F} \cup \Gamma_{S S}, \\
\nabla \mathbf{x}^{T} \cdot p n+\frac{\partial(\Delta \mathbf{x})}{\partial n}=0 & \text { on } & \Gamma_{F} .
\end{array}
$$

Physically, we can interpret these conditions as a zero bending moment on $\Gamma_{F} \cup \Gamma_{S S}$, and a balance of traction forces on $\Gamma_{F}$, due to bending and membrane contributions.

\subsubsection{Geometry of developable surfaces}

Here, we want to study a particular property of developable surfaces, namely their connection with the following class of surfaces.

Definition 3.1. (Ruled surface) A surface $S$ is ruled if through every point of $S$ there is a straight line that lies on $S$.

For more details regarding the geometry of developable surfaces, see [43].

\section{Gauss curvature}

In order to establish the connection, we first need to recall fundamental properties regarding the Gauss curvature of a surface.

Definition 3.2. (Gauss curvature) Let a surface be defined as the graph of the function $z=f(x, y)$. The Gauss curvature $K$ is the product of the principal curvatures, i.e., $K=\operatorname{det}\left(\nabla^{2} f\right)$.

The Gauss curvature $K$ is central to the bending-dominated deformation of surfaces due to the following classical result.

Theorem 3.4. (Theorema egregium) The Gauss curvature $K$ of a surface is invariant under local isometry.

For a proof, see [38]. In particular, developable surfaces are surfaces which are isometric to a plane, i.e., they are characterized by $K=0$. This is a local constraint, which geometrically means that locally any developable surface looks like a cylinder. 


\section{Smooth surfaces}

\section{Developable surfaces are ruled}

The result we are interested in is the following:

Theorem 3.5. In $\Re^{3}$, all developable surfaces are ruled.

Proof. We only sketch the idea of proof, which can be found in [4]. Consider a surface given as the graph of the function $z=f(x, y)$. The Theorema egregium implies that the determinant on the Hessian $\nabla^{2} f(x, y)$ is zero. It follows that it exists a parameter $\phi(x, y)$ such that $\nabla^{2} f(x, y)=\operatorname{tr}\left(\nabla^{2} f(x, y)\right) P(\phi(x, y))$, where $P(x, y)$ is a symmetric $2 \times 2$ matrix such that $\operatorname{det}(P)=0$ and $\operatorname{tr}(P)=1$. Since the partial derivatives of each component of $\nabla^{2} f(x, y)$ must permute, a compatibility condition involving only $\phi(x, y)$ is obtained. This condition is a PDE whose characteristic curves, along which $\phi=$ const, are the integral lines of $(\cos (\phi), \sin (\phi))$. This implies that the direction of the characteristic lines is given by $\phi$, i.e., they are straight lines. The integral lines can be lifted on to the surface and it can be shown that the surface is flat along their tangent and that these curves are straight lines spanning the whole surface.

The above theorem implies that the Theorema egregium, which yields a local constraint $K(x, y)=0$, has been transformed into a global constraint, i.e., the existence of straight rulings. This implies that globally a developable surface can be a cylinder, a cone, or a tangent developable surface, i.e., a ruled surface whose ruling consists of the tangents to a curve in $\Re^{3}$.

\section{Physics of developable surfaces}

Elastic plates and shells deform to avoid stretching whenever possible. Using the Föpplvon Karman kinematics, the Gauss curvature can be written in terms of the strain tensor as

$$
K=2 \frac{\partial^{2} \epsilon_{x y}}{\partial x \partial y}-\frac{\partial^{2} \epsilon_{x x}}{\partial y^{2}}-\frac{\partial^{2} \epsilon_{y y}}{\partial x^{2}} .
$$

However, the theory of elasticity of surfaces is not a straightforward application of the above geometrical concepts. The above theory relies upon the assumption that the surface is $C^{2}$, i.e., the tangent plane has a continuously differentiable normal vector. Unfortunately, a plate often minimizes the elastic energy by becoming singular, i.e., its radius of curvature can become comparable to the thickness $h$, forming a ridge or a singular cone point. Therefore, only local results away from singular points can be derived. For more details, see [4]. 


\section{Discrete surfaces}

Classically, shells are approximated by a piecewise polynomial representation of the surface, from which stretching and bending contributions can be measured. Such representation can be unique or differ for the two energies. Either way, this approach involves the use of curvilinear coordinates, which often occludes the intuition and leads to membrane locking. Instead, our framework consists of directly devising discrete counterparts of the smooth theories introduced earlier. The chapter is organized in the following sections:

1. We start by reviewing known discrete shell theories, translating them into the intrinsic Koiter theory presented in the previous chapter. In particular, we include a novel presentation of some popular models used in the geometry processing community, showing unexplored connections between them.

2. We continue by studying the approximation of curvature, surveying FE approximations of plates, for which bending and stretching deformations are uncoupled.

3. We briefly discuss stabilized FE in discrete membrane theories, focusing on the Enhanced Strain Method (EAS) and its relationship with Crouzeix-Raviart (CR) elements.

4. We discuss the limit case of discrete developable surfaces, for which a more efficient formulation can be used. We focus on the EBT discrete developable model proposed in [17], based on CR elements, performing a novel set of numerical experiments, which show that it possesses several failure modes.

5. We conclude by discussing classical FE for shells, providing a connection between discrete shell theories and lower-order FE, which will bring us to the central issue of membrane locking. In particular, we provide novel evidence that EBT suffers from locking and that it does not converge to the thin plate solution in the limit of refinement.

Throughout the chapter, we assume the discrete surface to be given as a simplicial 2-complex $\mathcal{T}_{h}$ [28], i.e., a set of triangles that satisfies the following conditions: (i) any face of a simplex from $\mathcal{T}_{h}$ is also in $\mathcal{T}_{h}$, and (ii) the intersection of any two simplices $\sigma_{1}, \sigma_{2} \in \mathcal{T}_{h}$ is a face of both $\sigma_{1}$ and $\sigma_{2}$.

\subsection{Discrete shell theories}

The discrete counterpart of the smooth intrinsic Koiter theory is built on the definition of three ingredients: a normal vector field, denoted with $\mathbf{n}$, and discrete first and second 


\section{Discrete surfaces}

fundamental forms, denoted with $\mathrm{I}_{f}$ and $\mathrm{II}_{f}$. As the notations suggests, we restrict our treatment to piecewise constant fundamental forms, defined at the faces $f$. In this case, the smooth energy (3.11) is written in the discrete energy as a sum over faces $f$ as

$$
W=\frac{t}{2} \sum_{f \in F} \bar{A}_{f}\left(\left\|\mathrm{I}_{f}-\overline{\mathrm{I}}_{f}\right\|_{K_{m}}^{2}+t^{2}\left\|\mathrm{II}_{f}-\overline{\mathrm{I}}_{f}\right\|_{K_{b}}^{2}\right),
$$

where $\bar{A}_{f}$ is the undeformed triangle area, and the norms encode the material properties, as seen in the previous chapters. We will come back to the problem of formulating discrete shell theories with piecewise linear strains in Chapter 6.

\subsubsection{Discrete quadratic forms}

In dimension two, any quadratic form is uniquely determined by its action on three linearly independent vectors. If these vectors form a triangle, the resulting expression becomes particularly simple.

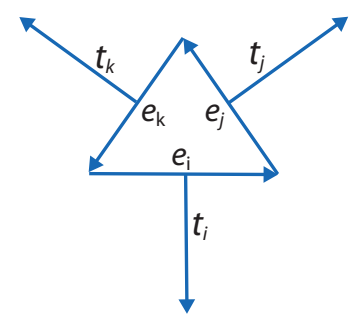

Figure 4.1.: Notations for the geometric representation of a triangle.

Writing a quadratic form in the basis $\mathbf{t}_{i}$ shown in Fig. 4.1, obtained by rotating clockwise the vector $\mathbf{e}_{i}$ in the plane of the triangle by $\pi / 2$, yields

$$
Q=\sum_{s=1}^{3} \mu_{s} \mathbf{t}_{s} \otimes \mathbf{t}_{s},
$$

where $\otimes$ denotes the outer product. From this, the action of the quadratic form on a vector, pulled-back on the undeformed surface, can be computed as

$$
Q_{i}:=Q\left(\mathbf{e}_{i}\right)=\mathbf{e}_{i}^{T} Q \mathbf{e}_{i}=\sum_{s=1}^{3} \mu_{s}\left(\mathbf{e}_{i}^{T} \mathbf{t}_{s}\right)^{2} .
$$

This expression can be simplified by noticing that

$$
\begin{aligned}
\left|\mathbf{e}_{i}^{T} \mathbf{t}_{i}\right| & =0, \\
\left|\mathbf{e}_{i}^{T} \mathbf{t}_{s}\right| & =\left\|\mathbf{e}_{i} \times \mathbf{e}_{s}\right\|=2 A .
\end{aligned}
$$

This set of equations can be solved to find the coefficients

$$
\mu_{i}=\frac{1}{8 A^{2}}\left(Q_{j}+Q_{k}-Q_{i}\right),
$$


where $(i, j, k)$ is a cyclic permutation of $(1,2,3)$ and $A$ is the area of the triangle. Therefore, we may represent a quadratic form $Q$ by the cyclic sum

$$
Q=-\frac{1}{8 A^{2}} \sum_{i=1}^{3}\left(Q_{i}-Q_{j}-Q_{k}\right) \mathbf{t}_{i} \otimes \mathbf{t}_{i} .
$$

Remark. Notice that the triangle depicted in Fig. 4.1 does not necessarily have to coincide with the canonical simplex defined by three vertices of the mesh, but the only requirement is that the condition $\sum_{i} \mathbf{e}_{i}=0$ holds. For example, the vector $\mathbf{e}_{i}$ can connect the edge midpoint of a triangle, as will be done in the following.

\subsubsection{Constant membrane triangles}

The discrete first fundamental form in built by choosing a set of positional DOFs. Since we have assumed I to be piecewise constant, which is is equivalent to considering piecewise constant membrane strains, we consider two possible options: vertices or edge midpoints. While producing two closely related approximations of membrane-dominated deformations, these two choices yield a dramatically different kinematic theory, as it will become clear in the bending-dominated scenario.

\section{Constant strain triangle (CST)}

With the choice of vertex-based DOFs, which we denote as $\mathbf{v}_{i}$, the vectors $\mathbf{e}_{i}$ in (4.3) represent the deformed edges of the mesh. In this case, the action of I when pulled back to the undeformed surface can be defined as

$$
\mathrm{I}_{i}:=\mathrm{I}\left(\overline{\mathbf{e}}_{i}, \overline{\mathbf{e}}_{i}\right)=\left\|\mathbf{e}_{i}\right\|^{2}=\left\|\mathbf{v}_{j}-\mathbf{v}_{k}\right\|^{2} .
$$

By rearranging the terms of the membrane energy, is possible to show [22] that the resulting model is equivalent to the piecewise linear $\mathrm{FE}$ approximation

$$
\begin{array}{rlrl}
\phi_{i}\left(\overline{\mathbf{v}}_{j}\right) & =1, & i=j, \\
\phi_{i}\left(\overline{\mathbf{v}}_{j}\right)=0, & i \neq j .
\end{array}
$$

In the case $\mathrm{II} \approx \overline{\mathrm{II}}=0$, the discrete first fundamental form reduces to the simplest convergent element for the 2D elasticity, formulated in terms of displacements. Unfortunately, as we shall see at the end of this chapter, this approximation suffers from severe membrane locking [17].

\section{Crouzeix-Raviart triangle (CRT)}

In Chapter 1, we have mentioned nonconforming elements as being the low-order equivalent of reduced integration. In the case of surfaces, nonconforming $\mathrm{FE}$ are known under the name of Crouzeix-Raviart (CR), which are built from edge-based DOFs $\mathbf{m}_{i=1 . . E}$ and piecewise linear basis functions inside each triangle, such that

$$
\begin{aligned}
\phi_{i}\left(\overline{\mathbf{m}}_{j}\right)=1, & i=j, \\
\phi_{i}\left(\overline{\mathbf{m}}_{j}\right)=0, & i \neq j,
\end{aligned}
$$




\section{Discrete surfaces}

where $\bar{m}_{i}$ is the undeformed position of the edge midpoint $e_{i}$. An interesting property of these elements [9] is that

$$
\mathbb{P}^{1} \subset \mathrm{CR},
$$

a fact that is often exploited in the context of stabilized FE, in which the quotient of the two spaces is penalized in order to control zero-energy spurious modes. In the intrinsic formulation, this discrete membrane model can also be obtained by choosing edge midpoints $\mathbf{m}_{i=1 . . E}$ as independent DOFs and defining the action of I when pulled back to the undeformed surface as

$$
\mathrm{I}_{i}:=\mathrm{I}\left(\overline{\mathbf{e}}_{i}, \overline{\mathbf{e}}_{i}\right)=\left\|\mathbf{e}_{i}\right\|^{2}=4\left\|\mathbf{m}_{j}-\mathbf{m}_{k}\right\|^{2} .
$$

From this formula, we notice that in a single triangle the strain is approximated with exactly the same accuracy as CST, but since $E \approx 3 V$, the resulting kinematic model is more flexible. We will see later in this chapter that this leads to zero-energy modes, having dramatic artifacts on the deformation.

\subsubsection{Constant curvature triangles}

The definition of a piecewise constant second fundamental form, relies mainly on the definition of the discrete normal field $\mathbf{n}$. We now explore two different choices: in the first case the surface normal vector field $n$ can be uniquely determined from the discrete surface, while in second one it uses an additional DOF in order to enrich the discrete configurational space of the shell, with the hope that the minimizer of the discrete energy can become closer to that of the smooth one.

\section{Constant curvature hinge $(\mathrm{CCH})$}

Since $\phi \in \mathbb{P}^{1}\left(\mathcal{T}_{h}\right)$, the surface normal is a piecewise discontinuous function. Since the bending energy relies on the computation of its gradient, it cannot be defined via pointwise differentiation but, as in discontinuous Galerkin methods, only in an integrated sense by considering its jump across inner edges.

Fortunately, such jump can be computed from geometrical considerations, as shown by [26]. First, the Gauss curvature is assumed not to vary under a bending dominated deformation, i.e., $K \approx \bar{K}$, so that the bending energy depends only on the mean curvature $H$. As a second step, $H$ is assumed to be piecewise constant over the dual cell around each edge $e_{i}$ of area $A_{e} / 3$, where $A_{e}$ is the sum of the areas of the two adjacent triangles. The integral of the mean curvature can be computed as

$$
\int_{\overline{\mathcal{S}}}(H-\bar{H})=(\theta-\bar{\theta})\|e\|,
$$

where $\theta_{e}$ is the dihedral angle between adjacent faces [26]. The pointwise mean curvature is obtained as the cell average

$$
H-\bar{H}=3(\theta-\bar{\theta}) \frac{\|e\|}{A_{e}} .
$$


By inserting this definition, the energy is immediately computed by integrating over the dual cell

$$
E_{b}=\int_{\overline{\mathcal{S}}}(H-\bar{H})^{2}=3(\theta-\bar{\theta})^{2} \frac{\|e\|^{2}}{A_{e}},
$$

where we have set the Young modulus to $E=1-\nu$ for simplicity. Originally the authors of [26] called the model Discrete Shells, but we believe the name $\mathrm{CCH}$ to be more appropriate to our presentation.

\section{Midedge shape operator (MSO)}

An alternative discrete Koiter model was developed by Grinspun et al. [25]. In this approach, the normal cannot be uniquely computed given the mesh, but it can rotate around each edge, as long as it remains orthogonal to it. This description leads to the so-called MSO shape operator on a triangle given in formula (6) in [25]

$$
\mathrm{II}=\sum_{i} \frac{\psi_{i}}{A l_{i}} t_{i} \otimes t_{i}
$$

where $\psi_{i}$ is the angle along $t_{i}$ between the face normal $n_{f}$ and the unknown surface normal $n_{m}$, i.e. $n_{m}=n_{f}+\psi \hat{t}_{i}$, and $t_{i}$ is the rotated edge vector. A straightforward calculation, similar to the one presented in the appendix of [25], gives the energy depending on the mean curvature:

$$
E_{b}=\frac{1}{2} \int H^{2}=\frac{1}{2} \int \operatorname{Tr} \mathrm{II}^{2}=\frac{1}{2 A} \sum_{i, j} \frac{\psi_{i}}{l_{i}} \frac{\psi_{j}}{l_{j}} T_{i j}=\frac{1}{2 A}\left\langle\frac{\psi}{l}, \frac{\psi}{l}\right\rangle_{T},
$$

where $T_{i j}=\left(t_{i} \cdot t_{j}\right)^{2}$. We will show that through a series of simplifying assumptions, it is possible to recover (4.17) from (4.19).

\section{Connection between CR and MSO}

We have already mentioned that the connection between the discrete first fundamental form and piecewise linear elements is well-known in the graphics community. However, the connection between MSO and the approximation $\mathbf{n} \in \mathrm{CR}$ is less known, but was already observed in the small deformation regime in [3], as will be explained in 4.2.2. First, let us observe that in a triangle the line segment connecting the midpoints of the edges $\overline{\mathbf{e}}_{j}$ and $\overline{\mathbf{e}}_{k}$ is parallel to the edge $\overline{\mathbf{e}}_{i}$ and has half its length, therefore the shape operator (4.18) can be written defining the pulled-back action

$$
\mathrm{II}_{i}:=\mathrm{II}\left(\overline{\mathbf{e}}_{i}, \overline{\mathbf{e}}_{i}\right)=2\left\langle\mathbf{n}_{j}-\mathbf{n}_{k}, \mathbf{e}_{i}\right\rangle,
$$

where $\langle\cdot, \cdot\rangle$ denotes the $\mathbb{R}^{3}$ standard inner product. Let $\left\{\psi_{i}\right\}$ denote the CR basis functions, taking value one at the midpoint of edge $\mathbf{e}_{i}$ and zero on all other edge midpoints. 


\section{Discrete surfaces}

Writing $\mathbf{n}=\sum_{i} \mathbf{n}_{i} \psi_{i}$ and using (3.11), for each triangle with (oriented) edges $\mathbf{e}_{i}, \mathbf{e}_{j}$, and $\mathbf{e}_{k}$, we obtain

$$
\mathbf{e}_{i}^{T} \mathrm{II} \mathbf{e}_{i}=\sum_{\alpha=i, j, k}\left\langle\mathbf{n}_{\alpha}, \mathbf{e}_{i}\right\rangle\left\langle\nabla \psi_{\alpha}, \mathbf{e}_{i}\right\rangle=2\left\langle\mathbf{n}_{j}-\mathbf{n}_{k}, \mathbf{e}_{i}\right\rangle,
$$

which the exact same expression as in (4.20). By inserting this into (4.7), we obtain

$$
\frac{1}{8 A^{2}}\left(Q\left(e_{j}\right)+Q\left(e_{k}\right)-Q\left(e_{i}\right)\right)=\frac{1}{4 A^{2}}\left\langle\mathbf{e}_{j}-\mathbf{e}_{k}, \mathbf{n}_{i}\right\rangle .
$$

Therefore, the second fundamental form is

$$
\mathrm{II}=\sum_{i} \cos \left(\frac{\pi-\psi_{i}}{2}\right) \frac{\left\|\mathbf{m}_{i}\right\|}{2 A^{2}} \mathbf{t}_{i} \otimes \mathbf{t}_{i}
$$

where $\mathbf{m}_{i}=\mathbf{v}_{i}-\mathbf{e}_{i}$ is the median of the triangle. Comparing (4.18) and (4.23), we see that the two are equal up to a factor $\left\|\mathbf{m}_{i}\right\| /\left\|\mathbf{h}_{i}\right\|$, where $\mathbf{h}_{i}$ is the height of the triangle from the vertex $\mathbf{v}_{i}$, and up to the small-angle approximation of the cosine.

\section{Connection between $\mathrm{CCH}$ and MSO}

We here present a novel interpretation of the connection between MSO and $\mathrm{CCH}$. The first step is to assume the surface normals not to be independent variables, i.e., they are computed from the positional variables as $n_{m}=n^{a v g}$, where $n^{a v g}$ is the average between the known face normals of two adjacent triangles, then:

$$
E_{b}=\frac{1}{2 A}\left\langle\frac{\theta}{2 l}, \frac{\theta}{2 l}\right\rangle_{T},
$$

where $\theta$ is the dihedral angle between two faces. Afterwards, we lump the matrix $T$, i.e., we substitute it with a diagonal matrix having the sum of each row as a diagonal entry, thereby loosing any directional information regarding the triangle geometry, obtaining $T_{i i}=12 l_{i}^{4}$, which implies

$$
E_{b}=\frac{3}{2 A} \sum_{i}\left(l_{i} \theta_{i}\right)^{2}
$$

As a final step, we obtain the desired result by smoothing (4.25), i.e., by associating to each edge the averaged energy

$$
E_{C C H}=\frac{A_{L} E_{b L}+A_{R} E_{b R}}{A_{L}+A_{R}},
$$

where the subscripts $L_{L}$ and ${ }_{R}$ indicate the left and right triangles, adjacent to an hinge. 


\section{Small angles}

We now extend the connection to the case of small angles, under the angle-bisecting assumption $n_{m}=n^{a v g}$. Let us define, in the notations of [26], $\xi:=n^{a v g} \cdot \tau=n^{a v g}$. $\left(n^{a v g} \times v\right)=0$, from which, we obtain a small-angle approximation of the MSO shape operator :

$$
\mathrm{II}=\frac{1}{A} \sum_{i} \frac{\hat{n} \cdot \tau}{t_{i} \cdot \tau} t_{i} \otimes t_{i} .
$$

where we have used that $t_{i}=l_{i} \hat{t}_{i}$. Additionally, observe that:

$$
\begin{aligned}
\hat{n} \cdot \tau & =\hat{n} \cdot\left(n^{a v g} \times v\right)=n^{a v g} \cdot(v \times \hat{n})=\left(n^{a v g} \cdot t\right), \\
A t \cdot \tau & =A t \cdot\left(n^{a v g} \times v\right)=n^{a v g} \cdot(v \times t) A=\left(n^{a v g} \cdot n\right) l^{2},
\end{aligned}
$$

where we used that $n=\hat{n} A$. We can write:

$$
\mathrm{II}=\sum_{i} \frac{n_{i}^{a v g} \cdot t_{i}}{l_{i}^{2}\left(n^{a v g} \cdot n\right)} t_{i} \otimes t_{i} .
$$

The corresponding energy is:

$$
2 E_{b}(u)=\int \operatorname{Tr} \mathrm{II}^{2}=12 A\left\langle\frac{n^{a v g} \cdot t}{n^{a v g} \cdot n}, \frac{n^{a v g} \cdot t}{n^{a v g} \cdot n}\right\rangle_{I d},
$$

where we used lumping of the matrix $T$ and that $T_{i i}=12 l_{i}^{4}$. Making use of

$$
n^{a v g}=\frac{\hat{n}+\hat{n}_{i}}{2}=\frac{A_{i} n+A n_{i}}{2 A} A_{i},
$$

where $\hat{n}_{i}$ is the unit face normal of the neighbor at the edge $i$, we now write

$$
E_{b}=6 A \sum_{i}\left(\frac{\left(A_{i} n+A n_{i}\right) \cdot t_{i}}{\left(A_{i} n+A n_{i}\right) \cdot n}\right)^{2} .
$$

We can convert it to hinge representation via the smoothing (4.26):

$$
\begin{aligned}
E_{b}= & \frac{\left\|\left(A_{L} n_{R}+A_{R} n_{L}\right) \cdot t\right\|^{2}}{A_{L}+A_{R}} \\
& \left(\frac{6 A_{L}^{2}}{\left\|\left(A_{L} n_{R}+A_{R} n_{L}\right) \cdot n_{L}\right\|^{2}}+\frac{6 A_{R}^{2}}{\left\|\left(A_{L} n_{R}+A_{R} n_{L}\right) \cdot n_{R}\right\|^{2}}\right),
\end{aligned}
$$

which simplifies to:

$$
E_{b}=\frac{6}{A_{L}+A_{R}}\left(\frac{\left(A_{L} n_{R}+A_{R} n_{L}\right) \cdot t}{n_{R} \cdot n_{L}+A_{R} A_{L}}\right)^{2} .
$$

Rearranging the terms, and using $t=n \times v$, we get:

$$
E_{b}=\frac{6}{A_{L}+A_{R}}\left(\frac{\left(n_{R} \times n_{L}\right) \cdot v}{n_{R} \cdot n_{L}+\left\|n_{L}\right\|\left\|n_{L}\right\|}\right)^{2}=\frac{6 l^{2}}{A_{L}+A_{R}}\left(\tan \frac{\theta}{2}\right)^{2},
$$




\section{Discrete surfaces}

which is a well-known second-order approximation of $\mathrm{CCH}$ valid for small angles [9]. While the above low-order models were developed in the context of geometry processing, engineers prefer to consider higher-order approximation of plates in order to introduce coordinates and to isolate the approximation of curvature, as we will see in the next section.

\subsection{Discrete curvature in plates}

As we have seen, in the regime of small deflections, bending and membrane deformations are uncoupled. It follows that the displacements can be split into in-plane and out-ofplane components, denoted with $u$ and $w$. While $u$ can be assumed to be, e.g., piecewise linear, the approximation of $w$ has remained a challenge to the $\mathrm{FE}$ community for several decades, as we summarize below.

\subsubsection{Discrete Kirchhoff-Love plate}

The central difficulty in proposing a discrete Kirchhoff-Love (KL) theory is the requirement $w \in H^{2}$, which does not arise naturally across elements using piecewise polynomials. This obstacle motivated the development of discrete Reissner-Mindlin (RM) theories based on mixed $\mathrm{FE}$, thereby lowering the required continuity to $H^{1}$.

The lowered regularity does not come for free, since a naïve choice of $\mathrm{FE}$ spaces approximating the RM theory, even when fulfilling $u \in H^{1}$, can lead to shear locking. As for the KL FE, the study of RM has occupied researchers for long time, eventually leading to uniformly convergent elements [3].

\section{$H^{2}$ elements}

The first natural approaches for achieving $H^{2}$ shape functions has been to increase the polynomial degree of the basis functions, hence increasing the number of DOFs, typically around 18 per triangle. This canonical direction has been followed by several authors, peaking with Irons. For more details, see [58] and the references therein.

However, the $H^{2}$ approach has been undermined by the result of Felippa [19], who showed that if one requires $H^{2}$ elements to represent constant curvature states - known as the patch test [58] - then only one of the following is possible: using a single polynomial over the element but admit higher order derivatives as corner DOFs, or permitting discontinuous derivatives at corners through non-polynomial assumptions.

Other authors have explored a completely alternative track, building nonconforming $H^{2}$ elements. For example, the Morley FE [58] builds a quadratic surface using 9 DOFs: positions at vertices and the normal component of $\theta$ ad edge midpoints, and has shown to perform surprisingly well. This element is particularly interesting, since it is the simplest one able to fulfill the patch test.

Recently, new developments were spurred by the use of subdivision surfaces by Cirak et al. [16]. This approach recursively subdivides the mesh in order to reduce the connectivity of each vertex to the case where a close formula to build $C^{1}$ splines is known. 


\begin{tabular}{|c|c|c|c|c|} 
Element & Year & DOFs & $C^{0}$ & $C^{1}$ \\
\hline Irons & 1969 & 18 & $\mathrm{Y}$ & $\mathrm{Y}$ \\
Morley & 1971 & 6 & $\mathrm{~N}$ & $\mathrm{~N}$ \\
DKT [6] & 1980 & 9 & $\mathrm{Y}$ & $\mathrm{N}$ \\
Subdivision & 2001 & $9^{*}$ & $\mathrm{Y}$ & $\mathrm{Y}$
\end{tabular}

Table 4.1.: Summary of FE for the KL theory; * denotes the need to build a finer mesh.

Following this recent trend, in the so-called isogeometric framework, by abandoning interpolation through vertices and polynomials in favor of non-uniform rational basis splines (NURBS), after half a century of research it has eventually become a trivial task to achieve any regularity on quadrilateral meshes [7]. Unfortunately, this is not the case for simplicial elements, which are the focus of this work.

\section{Discrete Kirchhoff Triangle (DKT)}

From the above discussion, we have intentionally omitted the DKT element, since its development shows how it is possible to successfully build discrete models from first principles, rather than by choosing basis functions, as done in standard FE.

To begin with, the rotations $\beta$ are assumed to be quadratic over the triangles, i.e., $\beta \in \mathbb{P}_{1}^{2}\left(\mathcal{T}_{h}\right)$, whose normal component varies linearly along each edge, giving a total of 9 coefficients $\beta_{i}$ to be determined per triangle. To compute them, 3 positions $w$ and 6 rotations $\theta$ are specified at vertices, thus resulting in 9 DOFs. Then, we introduce 6 trivial vertex constraints $\left(\beta_{i}^{x}, \beta_{i}^{y}\right)=\left(\theta_{i}^{y},-\theta_{i}^{x}\right)$, where $\theta_{i}^{x}$ indicates a rotation with respect to the $\mathrm{x}$-axis.

For the remaining conditions, the idea is to constrain a priori the discrete space, such that the condition $\beta=\nabla w$ is satisfied by construction on each edge. To do this, we assume that the displacement $w$ has a cubic variation along the edges, which can be expressed using a $1 \mathrm{D}$ cubic polynomial $x\left(w, \theta_{s}\right) \in \mathbb{P}^{3}$, where $s$ denotes the tangential component of $\theta$. By setting $\beta_{s}=\partial x / \partial s$, the 3 remaining coefficients are determined.

Referring to [6] for the explicit expression of the new basis functions, we conclude by observing that we have achieved the wanted regularity by building a space containing only discrete curl-free functions $\beta \in H^{1}$, i.e., $\mathbb{P}_{1}^{2}$ vector fields that are gradients of a function. Thus, this element is a special case of mixed FE, characterized by the possibility of eliminating a priori the shear stress from the unknowns.

\subsubsection{Discrete Reissner-Mindlin plate}

As anticipated, there exists a strategy to circumvent the $H^{2}$ regularity, starting from the RM theory with

$$
\begin{array}{ll}
u_{\alpha}=0, & \gamma_{\alpha}:=\sigma_{\alpha 3} \in \Gamma \subset L^{2}, \quad \alpha=1,2 \\
w \in W, & \theta \in V .
\end{array}
$$




\section{Discrete surfaces}

Brezzi, Arnold, Falk, and their collaborators, produced a vast literature regarding alternative convergent triplets of elements $\left(W_{h}, V_{h}, \Gamma_{h}\right)$, including lower and discontinuous elements [2]. Among these works, the first element shown to be uniform convergent to the KL theory as $t, h \rightarrow 0$, was developed by Arnold and Falk [3].

\begin{tabular}{|c|c|c|c|c|c|c|} 
Element & Author & Year & $V_{h}$ & $W_{h}$ & $\Gamma_{h}$ & $t \rightarrow 0$ \\
\hline T3D3 & Taylor [39] & 1990 & $\mathbb{P}^{2}$ & $\mathbb{P}^{1}$ & $\mathbb{P}^{0}$ & DKT \\
TLLL & Oñate [37] & 1994 & $\mathrm{CR}$ & $\mathbb{P}^{1}$ & $\mathrm{RT}$ & Morley
\end{tabular}

Table 4.2.: Summary of FE elements for the KL theory as a limit case of the RM theory.

In particular, we here mention two choices which have the property to correspond to a FE for the KL plate in the limit $t \rightarrow 0$, which are summarized in table 4.2 , where RT denotes the Raviart-Thomas elements [13]. The property of being able to express analytically the corresponding KL FE is quite remarkable, although in both cases such limit was discovered earlier. In Chapter 6 , we will be faced with a similar problem, i.e., given a limit kinematics as $t \rightarrow 0$, we will ask what is the corresponding energy which can generate it.

\subsection{Discrete membranes}

A canonical approach to formulate discrete shell theories, is to couple plate and membrane FE where the latter are obtained by approximating the smooth membrane theories presented at the end of the previous chapter. We have already seen that two possible definitions of piecewise constant first fundamental forms, are equivalent to the choices $\phi \in \mathbb{P}^{1}$ or $\phi \in \mathrm{CR}$ to discretize the membrane equations. Here, we concentrate on a more general approach, which contains $\mathrm{CR}$ as a special case. For mode details, we refer to section III.5 in [12].

\subsubsection{Enhanced assumed strains (EAS)}

Consider the Hellinger-Reissner model given in equations (3.46). As shown in [12], with the choice of discrete spaces

$$
V_{h} \subset V, \quad Q_{h} \subset Q, \quad \nabla V_{h} \subset Q_{h},
$$

one can eliminate the stresses from the equations and formulate the model in terms of positions, for which any conforming element is convergent.

On the contrary, if the discrete spaces are such that $\nabla V_{h} \not \subset Q_{h}$, a necessary requirement for the above problem to be well-posed is the fulfillment of Korn's second inequality. Such a condition requires that $\nabla x=0$ when $\epsilon=0$, i.e., infinitesimal rotations must induce stretching. For nonconforming elements, such a condition is not true in general, therefore the strain is corrected to $\epsilon=\nabla x+\tilde{\epsilon}$, where $\tilde{\epsilon}$ is orthogonal to $Q_{h}$. In other words, we have

$$
\nabla V_{h} \subset Q_{h} \oplus \tilde{E}_{h}
$$


so that the discrete Euler-Lagrange equations for (3.46) in terms of the displacement $u$ can be written as

$$
\begin{aligned}
\left(C^{-1} \sigma, \tau\right) & =(\epsilon(u), \tau) \quad \forall \tau \in \tilde{E}_{h}, \\
-(\sigma, \epsilon(v)) & =(f, v) \quad \forall v \in V_{h}, \\
\epsilon(u) & =\nabla u+\tilde{\epsilon}, \quad \tilde{\epsilon} \in \tilde{E}_{h} .
\end{aligned}
$$

In the case of CR elements, we have $V_{h}=\mathrm{CR}, \epsilon=\nabla u$, and $\sigma=C \epsilon$. Therefore, CR are unstable, meaning that Korn's second inequality is not fulfilled and the discretization is not convergent. In [18], it is shown that CR can be stabilized by choosing an appropriate $\tilde{E}$, consisting in refining the mesh and averaging the skew-symmetric component of the gradient onto the coarser mesh. By doing so, infinitesimal rotations of the finer mesh induce, on average, a stretching of the coarser mesh. This is also equivalent to enforce a weak symmetry of the stress tensor.

This correction is only one member of the family named the Enhanced Assumed Strains Methods (EAS), which has shown excellent results to tackle volumetric and shear locking. The advantage of this approach, rather than thinking in terms of projections between spaces, is an easier generalization to finite elasticity, as shown by [48]. However, it is computationally expensive, since the space $\tilde{E}_{h}$ is normally built using higher-order polynomials. More importantly, polynomial-based methods are poised to either generate membrane locking or zero-energy failure modes, depending on the deformation regime, as discussed at the end of this chapter.

\subsection{Discrete developable surfaces}

As shown by the asymptotic analysis performed in the previous chapter, in the case of non-inhibited surfaces, the vanishing thickness limit $t \rightarrow 0$ leads to consider developable surfaces. We start by discussing a suitable bending approximation, then we analyze in detail the discrete developable model proposed by [17], underlining its pitfalls.

\subsubsection{Discrete curvature}

As introduced earlier, under the assumptions of isometric deformations, the energy (3.51) is quadratic with respect to $\mathbf{x}$. This concept has been already exploited by [9], in order to precompute the hessian of the bending energy, thereby obtaining a significant speedup in the computations.

\section{$H^{1}$ formulation}

As seen in the previous chapter, in the case of developable surfaces, we need to discretize the quadratic bending energy (3.51). It can be shown [13] that the choice

$$
\mu=\nabla \cdot \theta=-\Delta \mathbf{x},
$$




\section{Discrete surfaces}

leads to the saddle-point problem

$$
\inf _{\mathbf{x} \in V_{0}} \sup _{\mu \in H^{1}} \frac{1}{2} \int_{\overline{\mathcal{S}}} \mu^{2} d s+\int_{\overline{\mathcal{S}}} \mu \Delta \mathbf{x} d s+\int_{\overline{\mathcal{S}}} f \mathbf{x} d s .
$$

If we consider small deformations, we can take $V_{0}=H_{0}^{1}$, then the solution is given by the Euler-Lagrange equations

$$
\begin{aligned}
\int_{\overline{\mathcal{S}}} \nabla w \nabla \psi d s=\int_{\overline{\mathcal{S}}} \mu \psi d s \quad \forall \psi \in H^{1}, \\
\int_{\overline{\mathcal{S}}} \nabla \mu \nabla \phi d s=\int_{\overline{\mathcal{S}}} f \phi d s \quad \forall \phi \in H_{0}^{1} .
\end{aligned}
$$

A discretization $(\mathbf{x}, \mu) \in V_{h} \times W_{h}$ of above system is convergent only if the inf-sup condition is fulfilled in the discrete setting, which reads [13]

$$
W_{h} \subset V_{h} .
$$

At first sight, the above formulation seems to involve only $H^{1}$ norms; at a closer inspection, however, we notice that the problem is ill-posed, since a priori $\mu$ could be a distribution and thus it cannot be integrated directly. Fortunately, by noticing that $f \in H^{-1}$, it follows that $\mathbf{x} \in H^{3}$, so $\nabla \mu$ is integrable.

However, the ill-posedness causes a degradation of the convergence rate, since the exact solution lives in a particular space embedded in $H^{1}$, which is slower to be approximated by $V_{h}$. Considering conforming elements, we have the following error estimates for the mixed discretization using twice the same space $W_{h}=V_{h}=\mathbb{P}_{1}^{k}$ :

- if the solution is $H^{4}$

$$
\begin{aligned}
& \left\|\mathbf{x}-\mathbf{x}_{h}\right\|_{0}=O\left(h^{1 / 2}|\ln (h)|\right) \text { for } k=1 \text { and } h \leq h_{0}[47] . \\
& \left\|\mathbf{x}-\mathbf{x}_{h}\right\|_{0}=O\left(h^{k+1}\right) \text { for } k \geq 2[13] .
\end{aligned}
$$

- if the solution is $H^{3}$

$$
\left\|\mathbf{x}-\mathbf{x}_{h}\right\|_{1}=O\left(h^{k}\right) \text { for } k \geq 2 .
$$

We thus conclude that $k=2$ is the optimal choice for the mixed formulation of the biharmonic problem. The poor choice $k=1$ is also known as the cotangent operator (COT) in geometry processing, since the stiffness matrix can be expresses using the cotangent of the inner angles of the mesh. A similar model, presented under the name of Isometric Bending Model (IBM) by [9], was shown to be equivalent to $\mathrm{CCH}$ for small angles. The IBM computes the shape operator from the nonconforming CrouzeixRaviart elements, projected it to the vertex DOFs. It remains an open question, thought, whether COT and IBM are equivalent or not. 


\section{CR formulation}

From definition (4.11)-(4.12), the surface is embedded as

$$
\mathbf{x}(u)=\sum_{e \in E} \mathbf{x}_{e} \phi_{e}(u)
$$

where $E$ is the total number of edges. The resulting bending model is computed using the mixed formulation (4.42), where $x, \mu \in \mathrm{CR}$. As for $\mathbb{P}^{1}$ elements, $\mu$ can be erased from the unknowns and, after several manipulations, the resulting model can be shown to be equivalent to

$$
E_{B}=\sum_{e \in E} \frac{3\|e\|^{2}}{A} \theta_{e}^{2}
$$

where $\theta_{e}$ is the angle between the edge vector rotated in the plane of the two triangles sharing $e$. We remark that there is a subtle difference between measuring $\theta_{e}$ using rotated edge vectors or between face normals, despite them being equivalent for specific kinematic assumptions, such that the one-angle hinge arising from CST. However, we do not explore further this topic.

\subsubsection{English-Bridson triangle (EBT)}

In the recent years, a discrete kinematic description of developable surfaces was proposed in [17], based on the CR elements for both membrane and bending energies. The resulting model was shown to perform visually well for open surfaces with free boundaries. However, in this novel analysis, we will show that there are several zero-energy stretching modes, which we call failure modes.

\section{Kinematics}

One can model developable surfaces by considering the thin limit of a discrete membrane theory. Above, we have already introduced the CST model, whose limit can be described by the constraints

$$
\left\|v_{i}-v_{j}\right\|=\left\|\bar{v}_{i}-\bar{v}_{j}\right\|, \quad \forall e=(i, j) \in E,
$$

where $v_{i} \in \Re^{3}$ is a vertex position and $E$ is the set of all edges of the mesh. This implies that an isometric deformation has $|E| \approx 3|V|$ constraints and $3|V|$ DOFs, so there is not enough freedom to avoid rigidification [17]. By considering the limit of CR elements, a different set of constraints is generated

$$
\left\|m_{i}-m_{j}\right\|=\left\|\bar{m}_{i}-\bar{m}_{j}\right\|, \quad \forall(i, j) \in f, \quad \forall f \in F,
$$

where $m_{i} \in \Re^{3}$ is an edge midpoint position, $F$ is the set of all faces of the mesh, and $(i, j)$ is a circular permutation of $(1,2,3)$. In this case, there are $3|F| \approx 6|V|$ constraints and $3|E| \approx 9|V|$ DOFs, leaving $3|V| \approx|E|$ DOFs to approximate any orthogonal deflection. Notice that this kinematic description implies that a vertex position is not not unique, i.e., its value differs when computed from its adjacent triangles. 


\section{Discrete surfaces}

However, in the case of planar isometric deformations, a more precise counting shows that there are $\left|E_{b}\right|$ redundant DOFs, namely, where $E_{b}$ is the set of boundary midpoints. In order to prevent zero-energy modes, these DOFs are removed by enforcing $C^{0}$ continuity at the boundary vertices. For visualization purposes, the method proposed by [17] tracks a ghost conforming mesh, whose vertex positions is computed by averaging the different values obtained from each adjacent nonconforming triangle.

\section{Failure Modes}

To verify in-plane and convex-body rigidity, we have devised the following set of benchmarks:

1. Glued plates. The aim is to test in-plane rigidity discarding boundary effects. Two plates are glued along their boundaries on top of each other, one vertex is fixed and gravity is applied tangentially to the surface. The only isometric deformation should be a rigid rotation, but we see in Fig. 4.2 that EBT allows for a wide range of in-plane artificial deformations.

2. Sphere. The top vertex of a sphere is fixed and gravity is applied vertically. The sphere is a convex body, therefore it is rigid according to Cauchy's theorem. We show in Fig. 4.3 that the membrane term of EBT is too flexible for closed surfaces, allowing for zero-energy deformations.

3. Cylinder Sagging. The two ends of the cylinder are fixed and gravity is applied orthogonally to its axis. An isometric cylinder should sag only up to second order, but we show in Fig. 4.4 that in-plane rotations of the triangles allow for a much greater elongation of the surface, even when the surface has boundary.
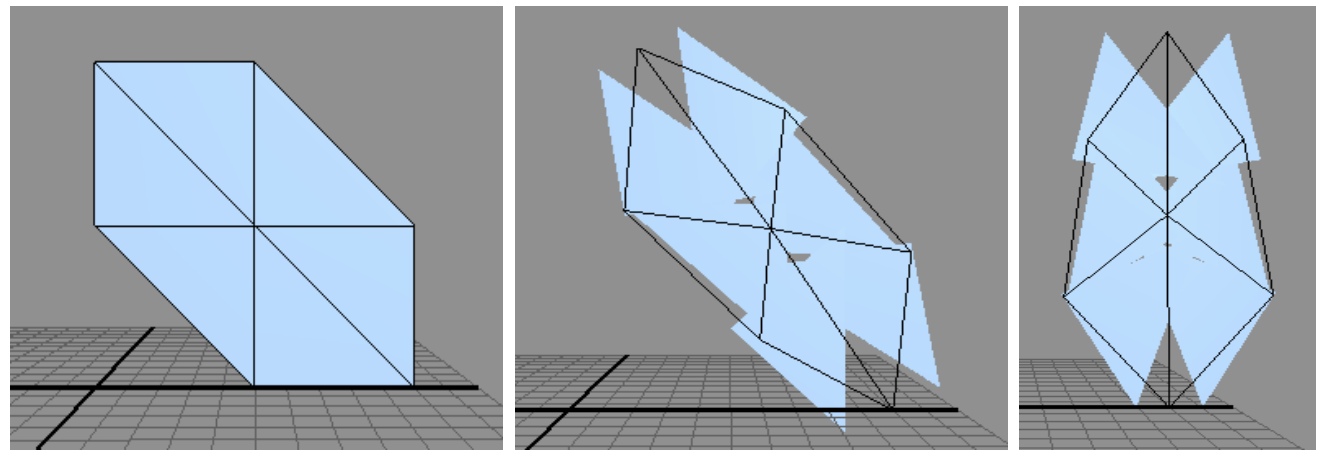

Figure 4.2.: Two hexagonal plates are glued along their boundaries on top of each other. One vertex is fixed, so the mesh should rotate rigidly under gravity. The wireframe indicates the ghost conforming mesh, as defined by [17].

All the above examples were solved using a dynamic solver with a relative stretching of $1 e-10$, enforced using the Fast Projection Method (FPM) by [23]. Interestingly, 

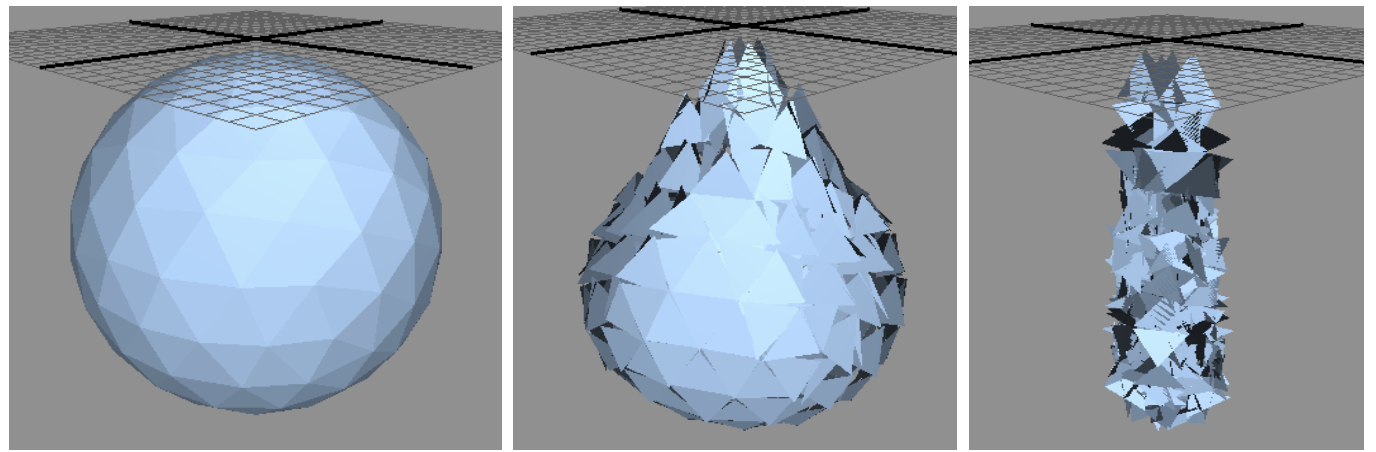

Figure 4.3.: Example of an isometric deformation of a sphere under gravity, according to nonconforming elements. The sphere is a convex body, so it is rigid.
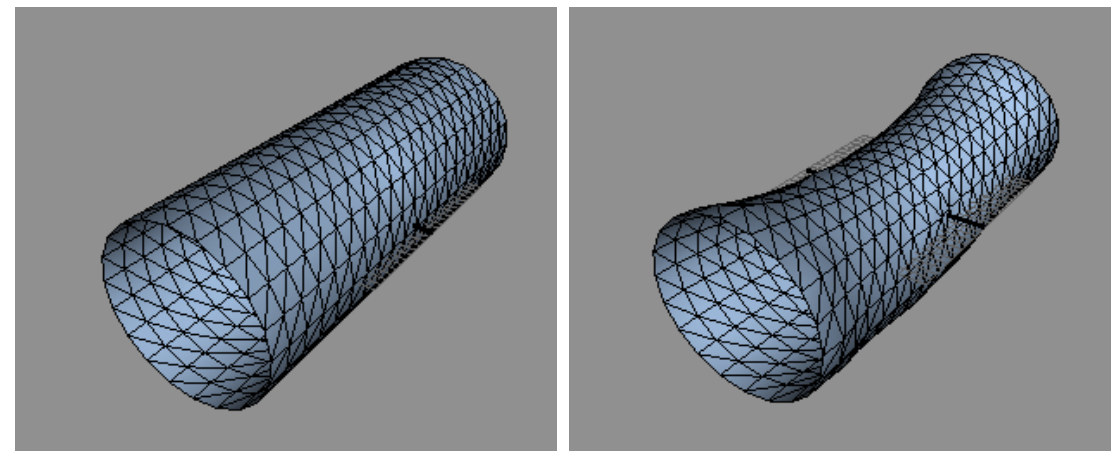

Figure 4.4.: The two end circles of a cylinder under vertical gravity are fixed. An isometric cylinder can sag only up to higher-order, but the nonconforming triangles can intrinsically rotate and thus allow for a larger deformation.

all failure modes are caused by in-plane rotations, as expected from an element violating Korn's second inequality. However, penalizing such rotations would restrict the kinematics to $\mathbb{P}^{1}$ elements, and therefore lead to an overconstrained system.

\subsection{Membrane locking}

In the literature of FE, two main approximations of the embedding $\phi$ can be found:

- Facet elements, i.e., $\phi \in \mathbb{P}^{1} \cap H^{1}\left(\mathcal{T}_{h}\right)$.

- Curved elements, i.e., $\phi \in \mathbb{P}^{k}\left(\mathcal{T}_{h}\right)$ for $k>1$.

Interestingly, some of the facet $\mathrm{FE}$ offer an independent formulation through the intrinsic discrete Koiter theory, as we have already seen for the CST model. While it is not clear - and also not the focus of this work - if this connection can be extended to other FE, the 


\section{Discrete surfaces}

previous section seems to suggest that the kinematics of discrete developable surfaces arising from facet elements is either under- or over-constrained. Therefore, we set to find an alternative description.

Unfortunately, the quest for an optimal discrete membrane theory has not enjoyed the same popularity as discrete bending and shearing theories did. The reasons are manifold: $H^{1}$ elements are easy to obtain, the membrane contribution vanishes for small deformations, and membrane locking is mild for the typical boundary conditions used in engineering. For coarse simulations, however, membrane locking can have a dramatic effect, which motivates the study of locking-free discrete membrane theories.

Normally, in the context of FE, two different approaches are used to discretize constrained systems:

- Look for reduced coordinates, using conforming elements. As we have seen, this cannot be done using linear elements. Unfortunately, the space of isometric deformations does not admit a polynomial representation, therefore also a pointwise exact approximation with higher-order FE is impossible.

- Underestimate the constraint, i.e., use reduced integration or mixed FE. As seen for EBT, this strategy can be very successful for bending-dominated scenarios, but it can fail dramatically for other asymptotic regimes.

There is a very subtle point hiding in the above discussion. While curved elements are generally $\mathrm{H}^{2}$, they rigidify inside each face, since they cannot represent curved states with a unit-speed parametrization, facet elements are always rigid in this sense, since curvature is a distribution located at edges. Therefore, facet elements rigidify due to the lack of compatibility of rigid rotations between the triangles, but they are always rigid inside.

\subsubsection{Curved elements}

As anticipated, a restrictive limitation of polynomials is that pointwise isometry results into the extermination of all nonlinear terms, which is precisely what engineers refer to, when they claim that higher-order membrane triangles have a stiffer behavior than lower-order ones, especially in regions of higher curvature. Therefore, one must employ reduced integration techniques.

For example, one can compute the metric distortion given by DKT bending elements [6], averaging each component of the strain tensor over each triangle. A quick counting, assuming Neumann conditions, shows that the number $d$ of free DOFs for a 2D deformation is a solution of

$$
\begin{aligned}
& 6|V|-3|F|+d=0, \\
& 2|E|-\left|E_{b}\right|=3|F|, \\
& |E|=3|V|,
\end{aligned}
$$

where $\left|E_{b}\right|$ is the number of boundary edges. The solution $d=|E|+\left|E_{b}\right|$ can be reduce to $d=0$ by prescribing $\left|E_{b}\right|$ additional conditions, which unfortunately lead to locking. 


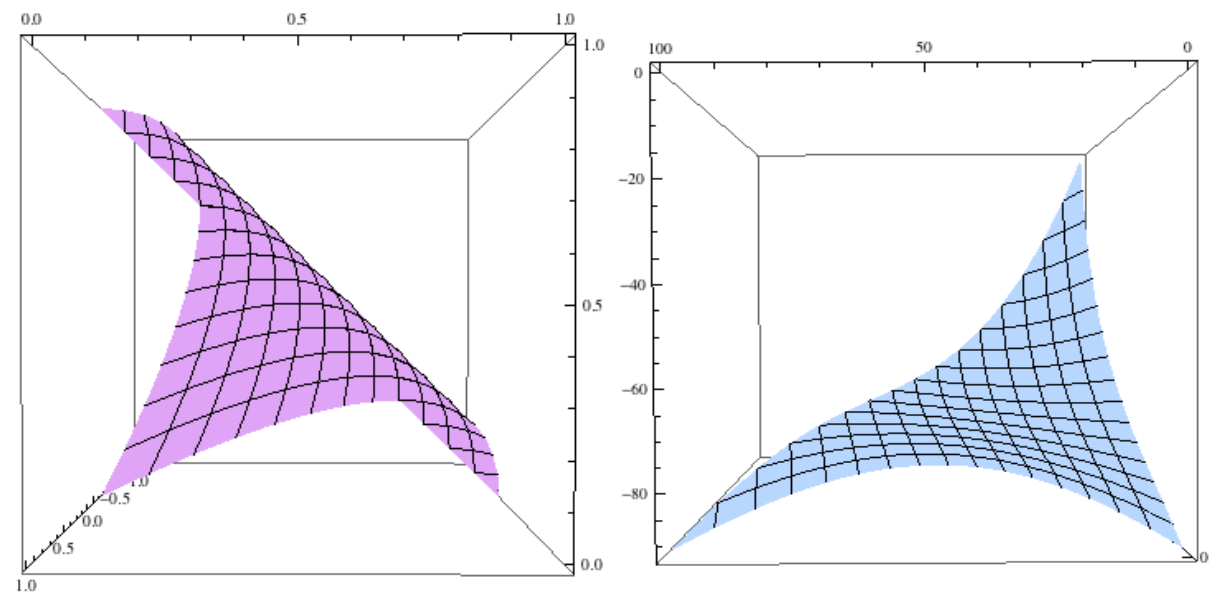

Figure 4.5.: Examples of kernel elements from the 2D membrane strain measure, when discretized using $\mathbb{P}^{2}-\mathbb{P}^{0}$ and DKT $-\mathbb{P}^{0}$ spaces respectively. The second one is known as a drilling mode.

As another example, one can extend to vector-valued functions the elements which are known to be convergent in the linear and scalar regime for shells, e.g., the quadratic element proposed in [1]. The resulting allowed in-plane flexibility for the DKT- $\mathbb{P}^{0}$ and the $\mathbb{P}^{2}-\mathbb{P}^{0}$ pairs can be seen in Figure 4.5 .

It is important to understand that such unwanted modes are intentionally allowed, in order to increase the freedom of representing bent states. To recover convergence to the minimizers of the total energy, employing the framework of Chapter 1, one must show that these modes are penalized by the bending energy, so that there are no overall zero-energy modes. Therefore, one needs both energies, which is the reason why FE are typically formulated for membrane- or bending-dominated regimes, but not for both.

From all these examples, we realize that reduced integration might not be a sensible approach for building a discrete shell theory, since there is an inherent difference between orthogonal and in-plane displacements, which is not captured. Notice in particular that, energetically, since the bending stiffness is much lower than the membrane, for the system it is always very convenient to introduce a bending deformation, if this is the only price which has to be paid in zero-energy stretching mode.

\section{MITC elements}

To conclude, we briefly discuss the "Mixed Interpolation of Tensorial Components" (MITC) element, which is claimed to be locking-free for a wide range of bedding-tomembrane ratios [40] . The key is a separate interpolation strategy for each component of the strain tensor, which is not computed directly from the displacements, as in the case of the discrete fundamental forms presented at the beginning of this chapter. In this sense, MITC is inspired by the EAS approach that we have outlined in the case of 


\section{Discrete surfaces}

discrete membrane theories.

A crucial difference between EAS and MITC is that, rather than augmenting the gradient of the displacement field with a carefully chosen space $\tilde{E}_{h}$, a set of tying points are selected, i.e., the equality $\epsilon(u)=\nabla u$ is enforced only at specific discrete locations and not in a projected sense, as done by FE. A detailed presentation and analysis can be found in [40] .

These elements perform quite well in bending-dominated scenarios. However, they are computationally expensive and less suitable for other asymptotic regimes. In Chapter 6 , we will make use of the tying point concept in order to improve the accuracy of our novel membrane element, proposing a low-order alternative to MITC elements, which is computationally cheaper and naturally suitable for any deformation regime.

\subsubsection{Facet elements}

We have already mentioned the connection between MSO, CCH, and CR elements. Even more interestingly, the MSO model is the intrinsic generalization of the Morley element to large displacements, for which there exists an equivalent $\mathrm{FE}$ formulation, introduced by van Keulen and Booij [56]. Geometrically, in the Kirchhoff case, the condition that $\mathbf{n}$ is a gradient field is defined in a discrete sense and enforced by construction.

However, $\mathbf{n}$ is the normal field of a (nonconforming) quadratic surface which never enters the computation of the first fundamental form. This is an example of the fact that different representations of the surface are needed for computing quantities of a different nature, such as its curvature and its metric. As we have seen, polynomials are very effective to compute the former, but they introduce artifacts in the latter. Therefore, in Chapter 6 we will discuss an alternative method based not on a representation of the surface, but on building a discrete kinematic theory from a given set of DOFs.

For example, since $\mathbf{n}$ is part of a gradient field of orthonormal frames at each point of a smooth developable surface, in the discrete case one must use a finite number of frames, which can be adapted to each face of the mesh. Orthonormality implies rigid triangles, so we additionally need compatibility conditions between the frames. Using $\mathbb{P}^{1}$ elements, such condition leaves the frames free to rotate about only one angle, which overconstrains the system. Using CR elements, with continuity only at edge midpoints, leaves three angles free. This is too much and, as we have seen, failure modes are generated.

In the next chapter, we will allow for zero-energy modes, but without any reduced integration, therefore there is no need for a complicated analysis involving the bending energy to have a bound over the zero-energy modes, since all these modes are geometrically admissible. The bending energy will eventually be introduced in our system, but only to obtain a smooth solution.

\section{Locking for English-Bridson}

Intuitively, the use of $\mathrm{CR}$ elements seems to be motivated from several angles: 

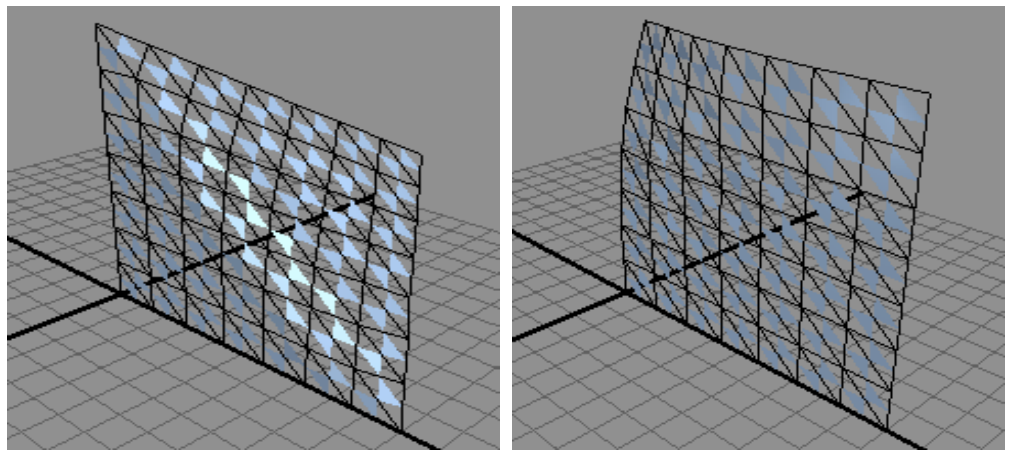

Figure 4.6.: Solution for two different orientations of the mesh. The edges of the conforming ghost mesh are in black, while the triangles connecting the edge midpoints are colored.
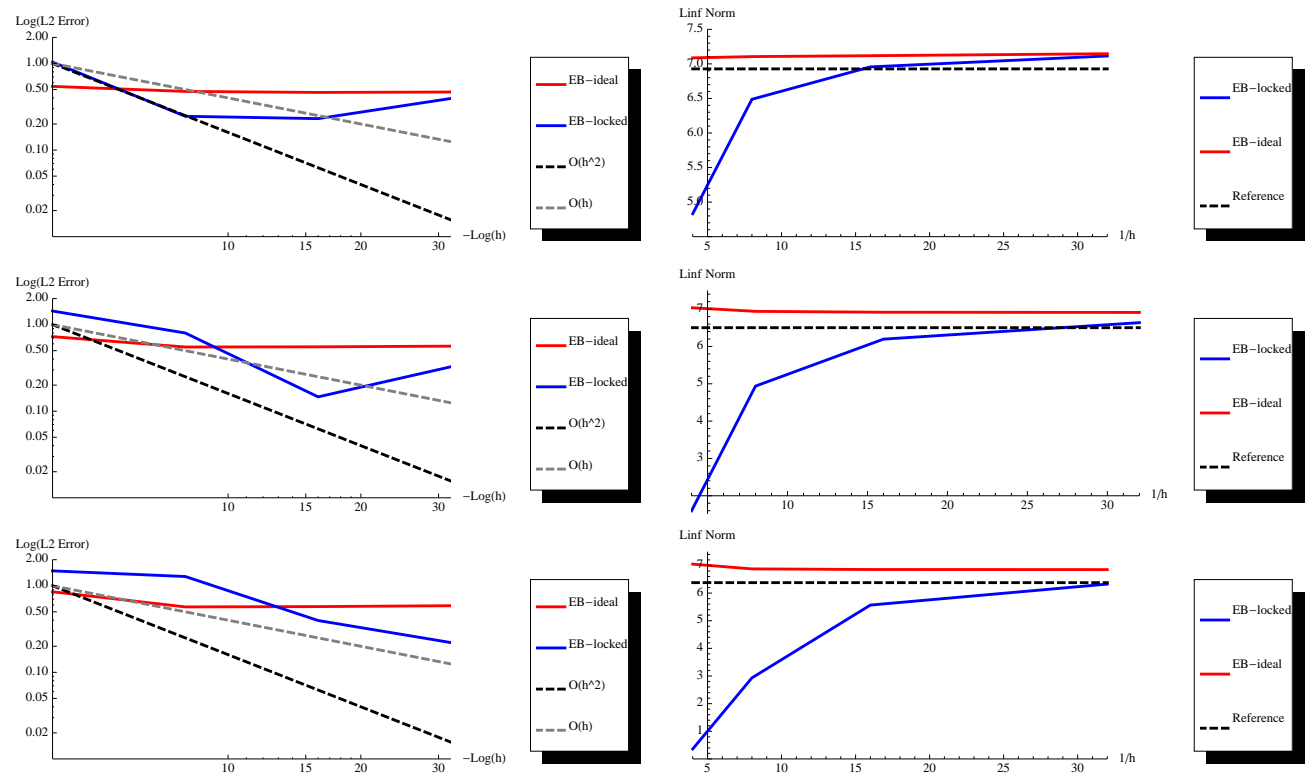

Figure 4.7.: English-Bridson. Left column: logarithmic plot of the $L^{2}$ error versus mesh resolution. Right column: $L^{\infty}$ norm versus mesh resolution. Each row corresponds to a different membrane stiffness $k=10^{2}, 10^{4}, 10^{6}$.

- analytic view: since (4.13), they are an enrichment of the configurational space, obtained by relaxing the $H^{1}$ regularity of linear elements.

- geometric view: by introducing some uncertainty on the positional approximation of the manifold of isometric deformations, namely, non-unique vertex positions, we can better approximate its tangent spaces. 
- physical view: by cutting the mesh along the edges, we introduce a limited amount of in-plane shear, which is intended to avoid rigidification, while not allowing for unbounded deformations.

However, we show numerically the novel result that the choice of a conforming boundary could induce locking. We consider a square plate fixed at two edges and a regular mesh, consisting of a regular grid where each square is subdivided in two triangles. The same solution should be obtained when all the diagonals are aligned or orthogonal to the bending axis.

From Fig. 4.7 and 4.6, we note that not only locking is present, but the solution does not converge under refinement. For coarse meshes, the locking effect is dominant due to the conforming boundary. Under refinement, however, the consistency error becomes more important and it ultimately undermines convergence in the limit or refinement. 


\section{Adaptive surfaces}

In this chapter, we consider the problem of automatically finding the best discrete kinematics for approximating developable surfaces. We ask the following requirements:

- It should be robust under any deformation regime, i.e., no reduced integration.

- It should be efficient, i.e., no higher-order elements.

- It should be simple, i.e., no combinatorial changes in the mesh.

Notice that the reduced integration and higher-order elements always go together in the traditional literature of FE, while the third one is not a limitation but a choice, since it is possible to extend the proposed methods to incorporate adaptation in the combinatorics, to further improve their performance.

The chapter starts by presenting a local version of r-adaptivity, then it continues with the global r-adaptive case, including a detailed presentation of the dynamic case, concluding with a discussion of rh-adaptivity. To our knowledge, this is the first study of r-adaptivity in the context of shells.

\subsection{Edgepoints}

In the first part of this chapter, we consider an element based on a single subdivision of triangles with movable edge midpoints ("edgepoints"), which can slide along their respective edges in the undeformed configuration. For this reason, we baptize this element the Eulerian-Lagrangian Triangle, or ELT. This element resembles the $\mathbb{P}^{1}$ iso $\mathbb{P}^{2}-\mathbb{P}^{1}$ elements, used in CFD and shown in Fig. 5.1. To our knowledge, such elements have never been explored outside the Eulerian framework.
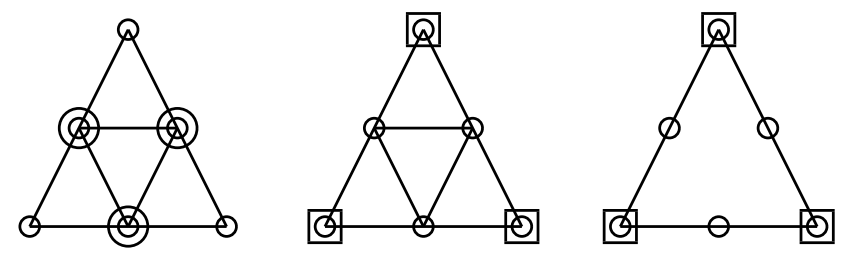

Figure 5.1.: DOFs for ELT, $\mathbb{P}^{1}$ iso $\mathbb{P}^{2}-\mathbb{P}^{1}$, and higher-order $\mathbb{P}^{2}-\mathbb{P}^{1}$ elements. The double circles indicate primal DOFs (positions) in the deformed and undeformed configurations, while the squares indicate dual DOFs (pressure or strains). 


\section{Adaptive surfaces}

In our case, rather than assuming a piecewise linear pressure field, we assume a piecewise constant strain. In the case of edgepoints being fixed in the undeformed configuration, the ELT element is equivalent to the use of $\mathbb{P}^{1}-\mathbb{P}^{0}$ elements on a refined mesh. However, if the deformed subdivided triangle remains isometric to the undeformed one, the effect of the edgepoint sliding along the edges is to relax the strain, making it constant (and not piecewise constant) over the entire coarse element.

Geometrically, the deformation represented by ELT is a conforming vertex-based mesh with movable hinges, created by the edgepoints, about which the sub-triangles can rotate rigidly, such that by unfolding the deformed triangle to the plane, it is congruent to the undeformed one. Such discrete representation of a developable surface can be intuitively motivated in different ways:

- analytic view: a given mesh represents a given parametrization of the domain; to enrich the configuration space, it is necessary to re-parametrize it, which can be done triangle-wise subdividing them via movable nodes.

- geometric view: any ruling of a developable surface can exactly be captured on a given mesh by placing new nodes on the intersections between the ruling and the edges of the mesh.

- physical view: locking is caused by an overestimation of the membrane energy, which is minimized by splitting each triangle with movable nodes and let their position relax such that the triangle achieves a constant strain state.

Given six nodes per triangle, we have an approximation of the configurational space of isometric deformations of quadratic surfaces. Notice that the edgepoints do not interpolate such quadratic surface, but approximate its freedom of movement. Morally, we have swept membrane distortion under the rug of the distributional discontinuity located across hinges.

Importantly, we observe that the proposed model is automatically rigid in-plane, since it is equivalent to have infinite undeformed conforming meshes available at the same time, which are known to be all in-plane rigid. In fact, while we devised this model from physical and geometric considerations, afterwards we found it to be a particular member of r-adapivity methods, as will be further investigated in this chapter.

\section{Kinematics}

Let us denote the undeformed and deformed vertices and midpoints with $\bar{v}_{i}, v_{i}$ and $\bar{e}_{i}, e_{i}$, as shown in Fig. 5.1. Each edgepoint is allowed to slide along its edge with the introduction of a new DOF denoted with $s$

$$
\begin{gathered}
\bar{e}_{i}=s_{i} \bar{v}_{j}+\left(1-s_{i}\right) \bar{v}_{k}, \quad \forall i=(j, k) \in E, \\
0 \leq s_{i} \leq 1 .
\end{gathered}
$$

Given edgepoints and vertices, the constant strain triangle (CST) energetic contribution can be computed on each of the four sub-triangles, noticing that the undeformed configuration is not constant but depends on $s$. To avoid degenerate triangles, we correct 


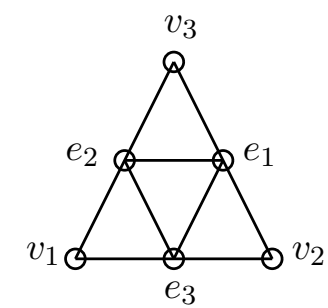

Figure 5.2.: DOFs labeling for the ELT triangle.

(5.2) to

$$
\begin{gathered}
\epsilon \leq s_{i} \leq 1-\epsilon, \quad \forall i \in E \\
0 \leq \epsilon \leq 1 / 2,
\end{gathered}
$$

Obviously, formula (5.3) reduces to (5.2) as $\epsilon \rightarrow 0$. In order to enforce such inequality, we add to the energy the following penalty term:

$$
\mu\left(\min \left(s_{i}, \epsilon\right)^{2}+\min \left(1-s_{i}, \epsilon\right)^{2}\right),
$$

where $\mu$ is a penalty parameter.

If the coarse triangle remains almost isometric, in particular it remains a triangle. Therefore, in order to allow for faster computations, it is possible to use this additional constraint, together with the constant strain assumption in order to compute the CST energy for the whole triangle at once from its deformed piecewise linear edges. However, we do not explore further optimizations of this kind in this context.

\subsubsection{Degenerate triangles}

We have already anticipated that the choice of (5.3) is tailored to exclude degenerate triangles from the kinematics. Here, we argue that we are forced to make such a choice. We start by illustrating several techniques to avoid the issues encountered in the case of vanishing triangles.

Let us start by presenting a dual formulation which results in an algorithm which: (i) does not suffer from ill-conditioning, (ii) is as expensive as the primal formulation if triangles are not degenerate, (iii) offers a smooth transition between primal and dual formulations, and (iv) is suitable for discrete energies of the form:

$$
E(u)=\frac{1}{2 h(u)}\langle\epsilon(u), \epsilon(u)\rangle_{T(u)},
$$

where $\langle u, v\rangle_{T}=u^{T} T v$ is the discrete $L^{2}$ inner product with metric $T$. Additionally, we require that the gradient of $E(u)$ does not blow up at finite energies for any $u$ which, as we will see, is a very strong requirement. We remark that, while handling the computation of quantities of the form $0 / 0$ is a numerical problem, a blow-up of the gradient is a modeling issue. 


\section{Adaptive surfaces}

The total energy of the system is given by:

$$
J(u)=\frac{1}{2}\langle\Delta u, \Delta u\rangle_{M}+\frac{1}{2 A^{3}}\langle d L, d L\rangle_{K},
$$

where $M$ is the mass matrix, $K$ is the stiffness matrix, and $d L_{i}=l_{i}^{2}-\bar{l}_{i}^{2}$. In order to dualize it, let us define $\sigma:=A^{-3} d L$. By inserting it into $J(u)$, we obtain:

$$
L(u, \sigma)=\frac{1}{2}\langle\Delta u, \Delta u\rangle_{M}+\langle\sigma, d L\rangle_{K}-\frac{1}{2}\left\langle\sigma, A^{3} \sigma\right\rangle_{K},
$$

where $\sigma$ acts as a multiplier, enforcing the constraint $c(u)=d L-A^{3} \sigma=0$. This problem is now well conditioned even for $A=0$, and it offers a smooth transition to $d L=0$ as $A \rightarrow 0$. We now devise an algorithm with the following goals:

- Do not increase the number of variables in the Newton's iteration.

- Deal with minimization problems only, for efficient line search.

To this aim, we employ the augmented Lagrangian (AL) technique, which is constructed by adding a penalty $\mu$ to the above functional, in order to help the constraint enforcement while being zero at the solution. We obtain the following augmented functional:

$$
L_{A}(u, \sigma, \mu)=L(u, \sigma)+\frac{1}{2 \mu}\langle c(u), c(u)\rangle_{I},
$$

where $I$ is the identity matrix. We use the standard update rule for the multipliers [36], with a clever choice of $\mu$ for some small $\xi$ :

$$
\begin{aligned}
u_{k+1} \leftarrow \min _{u} L_{A}\left(u, \sigma_{k}, \mu_{k}\right), & u_{0} & =\bar{u}, \\
\sigma_{k+1}=\sigma_{k}+\frac{1}{\mu_{k}} c\left(u_{k}\right), & \sigma_{0} & =0, \\
\mu_{k+1}=\mu_{k}, & \mu_{k} & =\max \left(\xi, A^{3}\left(u_{k}\right)\right),
\end{aligned}
$$

where $u_{k+1}$ is computed using Newton's method with line search.

If $A^{3}>\xi$, then $\sigma$ becomes a fixed point of the iterative update. To see this, note that $\mu_{k}=\mu_{k}\left(u_{k}\right)$ but $\sigma_{k}=\sigma_{k}\left(u_{k-1}\right)$. Thanks to this choice for $\mu$, in the case of no degenerate triangles $A(u)>\tilde{A}$, we obtain that $L_{A}\left(u, 0, A^{3}\right)=J(u), c\left(u_{1}\right)=d L\left(u_{1}\right)$, and $\sigma_{1}=d L / A^{3}$. It follows that again $L_{A}\left(u, \sigma_{1}, A^{3}\right)=J(u)$, and without any additional iteration we get $u_{2}=u_{1}$ and $c\left(u_{2}\right)=0$, so the method is as expensive as using directly the original primal energy $J(u)$.

\section{Membrane energy}

The CST membrane energy for a triangle with undeformed and deformed edge lengths $\bar{l}_{i}$ and $l_{i}$, can be written by integrating the square of the membrane strain as:

$$
E_{m}=\frac{A}{2(8 A)^{4}}\left\|\sum_{i=1}^{3}\left(t_{j} \otimes t_{k}+t_{k} \otimes t_{j}\right) d L_{i}\right\|^{2}=\frac{1}{2 A^{3}}\langle d L, d L\rangle_{K},
$$


where $K$ is the CST stiffness matrix. Writing $A=b h / 2$ and assuming that undeformed and deformed edges are of the same order of magnitude, we can distinguish the following degenerate cases:

- $b \rightarrow 0$, which implies that $A=o(b)$ and $\langle d L, d L\rangle_{K}=o\left(b^{4}+b^{2}\right)$. Combining the two, we obtain $E_{m} \rightarrow \infty$. Since the solution is the minimum of a positive energy, this case is excluded by the physics of the system.

- $h \rightarrow 0$, which implies that $A=o(h)$ and $\langle d L, d L\rangle_{K}=o(1)$. Combining the two, we obtain again that $E_{m} \rightarrow \infty$, which is excluded by the physics of the system.

- $b, h \rightarrow 0$, which implies that $A=o(b h)$ and $\langle d L, d L\rangle_{K}=o\left(b^{4} h^{4}\right)$. Combining the two, it follows that $E_{m} \rightarrow 0$, as one would expect if the domain reduces to a single point.

From the above analysis, it is clear why we need to apply the algorithm we have outlined earlier to handle the third case. To see this, notice that a naïve correction, e.g., by defining a modified area $\tilde{A}=\max \left(A_{0}, A\right)$, where $A_{0}$ is a small nonzero threshold, it is not possible, since it would alter the other two asymptotic regimes.

\section{Bending Energy}

We now concentrate on the constant hinge triangle $(\mathrm{CCH})$ bending model presented in the previous chapter, the simplest bending model available in the literature, which we write as

$$
E_{b}(e)=\frac{3}{A_{e}}\left\langle\theta_{e}\|e\|, \theta_{e}\|e\|\right\rangle_{I d}=\frac{\|e\|}{\|* e\|}\left\langle\theta_{e}, \theta_{e}\right\rangle_{I d} .
$$

When we subdivide all triangles, by connecting the edgepoints, we have three additional contributions for each face, coming from the hinges created by the edgepoints. We now analyze all possible cases as the variables $\left(\theta_{e},\|e\|,\|* e\|\right)$ go to zero in equation (5.10), assuming to be able to compute $\theta_{e}$, which is not always possible. Consider the three inner hinges of a single triangle, see Fig. 5.3 (left). There are the following cases:

- If we slide a single edgepoint into a vertex, then $A \neq 0$, so $E<\infty \forall \theta$.

- If we slide two edgepoints into different vertices, then we have $A=0$ and $\|e\| \neq 0$ at two hinges. To have a finite energy, it must hold $\theta \rightarrow 0$ with at least the same order. Thus, the bending energy does not allow two edgepoints to slide into different vertices, while keeping a curved state.

- If we slide two edgepoints into the same vertex, then we have $\|e\|=A=0$, but $\|* e\| \neq 0$. This means that $E=0 \forall \theta$.

Consider now two triangles bent at an angle $\theta=\theta_{0}>0$, see Fig. 5.3 (right). The main hinge will be subdivided in two parts by its edgepoint, and we will focus on only one of these two parts, and its two adjacent subtriangles. For example, we can consider the right vertex (in red), together with its three adjacent edgepoints (in pink). 


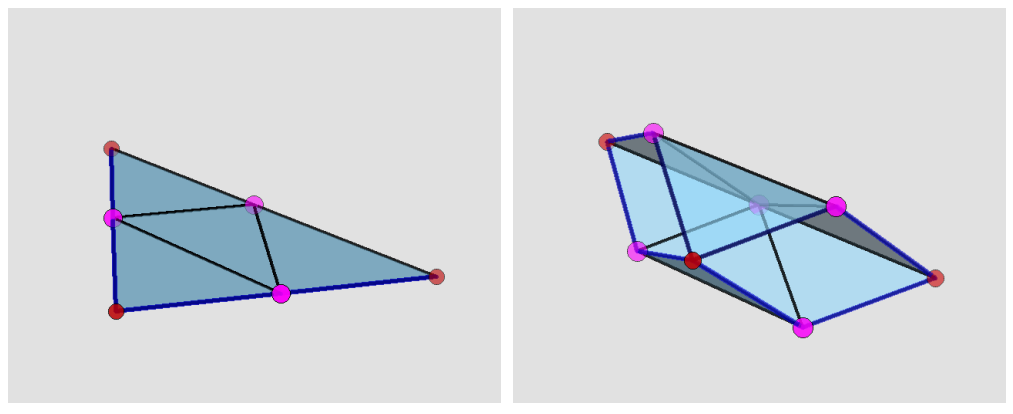

Figure 5.3.: (a) Single triangle, (b) Hinge. Edgepoints are in pink, vertices in red.

- If we slide one of the two flap edgepoints (those not on the segment connecting the hinge vertices) towards the hinge vertex, then $A \neq 0$, so $E<\infty \forall \theta$.

- If we slide both flap edgepoints towards the hinge vertex, then $A=0$ and $\|e\| \neq 0$. To have a finite energy, it must hold that $\theta \rightarrow 0$ with the same order. So, the bending energy does not allow two edgepoints to slide into the same vertex, while keeping a curved state.

- If we slide the hinge edgepoint towards a vertex, while keeping fixed the other edgepoints, then $A=\|e\|=0$ but $\|* e\| \neq 0$, so $E=0 \forall \theta$.

- If we slide all three edgepoints towards the same vertex, then $\|e\|, A,\|* e\|=0$, so $E=0 / 0$. By combining the speed at which $\|e\|$ or $\|* e\|$ are going to zero, i.e., the speed at which the edgepoints are moving along their edges, we can obtain all the previous situations.

The situation becomes even more complicated for the forces, as we show in the following propositions.

Proposition 5.1. If the half dual of one edge has zero length, then the CCH forces can blow up at finite energies.

Proof. Consider $E=\theta^{2}\|e\|^{2} / A$. Then the forces are $F=-\nabla E=\nabla\left(\theta^{2}\right)\|e\|^{2} / A+$ $\nabla\left(\|e\|^{2} / A\right) \theta^{2}$. Since we assumed finite $\|e\|, A$, and $\theta$, we only consider $\nabla\left(\theta^{2}\right)=2 \theta \nabla \theta$. We know that if $\theta=\operatorname{atan} 2(x, y)$, then $\nabla \theta(x, y)=(-y, x) /\left(x^{2}+y^{2}\right) \cdot \nabla(x, y)$. Since we have $x=n_{L} \cdot n_{R}$ and $y=\left(n_{L} \times n_{R}\right) \cdot e$, where $e$ is the edge, which both go to zero when either one of the vector areas $n_{L}$ or $n_{R}$ go to zero. It remains only to show that one component of $\nabla(x, y)$ does not vanish. Assume that $\left\|n_{L}\right\| \rightarrow 0$ and consider $\|\nabla x\|=\left\|\nabla\left(n_{L} \cdot n_{R}\right)\right\| \geq\left\|n_{L} \cdot \nabla\right\|\left\|n_{R}\right\| \geq\left\|\nabla n_{L}\right\|\left\|n_{R}\right\|>0$, which completes the proof.

Proposition 5.2. If the half dual of one edge has zero length, then the CCH forces do not blow up at finite energies, if the triangle having zero height has a neighbor along a non-degenerate edge. 
Proof. Consider two hinges $\left(\left\|e_{1}\right\|, A_{1}, \theta_{1}\right)$ and $\left(\left\|e_{2}\right\|, A_{2}, \theta_{2}\right)$, sharing a triangle with degenerated area. With the approximation $\|e\|:=\left\|e_{1}\right\| \approx\left\|e_{2}\right\|$, the energy is $E=$ $\left(\theta_{1}^{2} / A_{1}+\theta_{2}^{2} / A_{2}\right)\|e\|^{2}$. Then the forces are $F=-\nabla E=\nabla\left(\theta_{1}^{2}\right)\|e\|^{2} / A_{1}+\nabla\left(\|e\|^{2} / A_{1}\right) \theta_{1}^{2}+$ $\nabla\left(\theta_{2}^{2}\right)\|e\|^{2} / A_{2}+\nabla\left(\|e\|^{2} / A_{2}\right) \theta_{2}^{2}$. Since we assumed finite $\|e\|, A_{i}$, and $\theta_{i}$, we only consider $\nabla\left(\theta_{1}^{2}\right)\|e\|^{2} / A_{1}+\nabla\left(\theta_{2}^{2}\right)\|e\|^{2} / A_{2}=2\|e\|^{2}\left(\theta_{1} \nabla \theta_{1} / A_{1}+\theta_{2} \nabla \theta_{2} / A_{2}\right)$. With the choice $\theta_{1}=A_{1} \theta /\left(A_{1}+A_{2}\right)$ and $\theta_{2}=A_{2} \theta /\left(A_{1}+A_{2}\right)$, where $\theta$ is the total angle between the non-degenerated faces with areas $A_{1}$ and $A_{2}$, it follows that the gradient is finite.

To bypass the use of the edgepoint mesh $\mathcal{T}_{e}$ for the computation of the bending energy, we now take a different perspective and observe that the normal $n_{i}$ to each subface $i=1 \ldots 4$ of a triangle is a discontinuous function, being constant inside each edgepointbased subtriangle. One possible idea to tackle the degeneracy issue, is to use the midpoint mesh $\mathcal{T}_{m}$, thereby looking for $n \in \mathbb{P}^{0}\left(\mathcal{T}_{m}\right)$.

The natural way to transfer $n_{i}$ to the midpoint-based mesh is via $L^{2}$ projection, i.e. by means of a weighted average of the normals:

$$
\tilde{n}_{i}=\bar{A}_{i} \sum_{j=0}^{4} \alpha_{i j} \frac{A_{j}}{\bar{A}_{i}} \frac{n_{j}}{\left\|n_{j}\right\|}=\sum_{j=0}^{4} \alpha_{i j} n_{j},
$$

where $\alpha_{i}$ is the fraction of the edgepoint-based triangle contained into the midpointbased one. Consider the intersection between $e_{2} e_{0} e_{1}$ and $v_{1} m_{2} m_{0}$. Its area $\alpha_{1} A_{1}$ can be expressed as:

$$
\alpha_{1} A_{1}=A_{e}
$$

where $A_{e}$ is the area of $e_{0} e_{2} e_{1}$.

The advantage of this formulation, is that by assuming nearly isometric deformations, we have $l_{i} \approx \bar{l}_{i}$ and $A_{i} \approx \bar{A}_{i}$, therefore the $\mathrm{CCH}$ formula simplifies to:

$$
E_{b}=3 \sum_{i=0}^{8} \theta_{i}^{2} \frac{\bar{l}_{i}^{2}}{\bar{A}_{i}}
$$

where barred quantities refer to the undeformed configuration.

However, as we have seen in Chapter 2, the approximation of piecewise constant functions using piecewise constant functions defined on a different mesh, causes an irreversible loss of angle information, and it therefore yields undesirable zero-energy modes, unless a different jump is introduced. Unfortunately, there is no clear geometric intuition for doing so.

Therefore, we decide to use equation (5.3) in order to avoid degenerate triangles, by creating a forbidden area around vertices inside which the midpoint cannot lie, which we explore numerically in the following.

\subsubsection{Numerical experiments}

In general, if we assume smooth deformations, then the smooth rulings are not single lines but sets of parallel lines, so the edgepoints are free to adjust in order to approximate 


\section{Adaptive surfaces}

this set with a finite number of discrete rulings, depending on the mesh resolution. In other words, there is no explicit condition requiring edgepoints and vertices to overlap. In the case of nonsmooth deformations, the sets collapse to single lines, or to a set of line intersecting to a point, so there is no freedom in placing discrete bending lines.
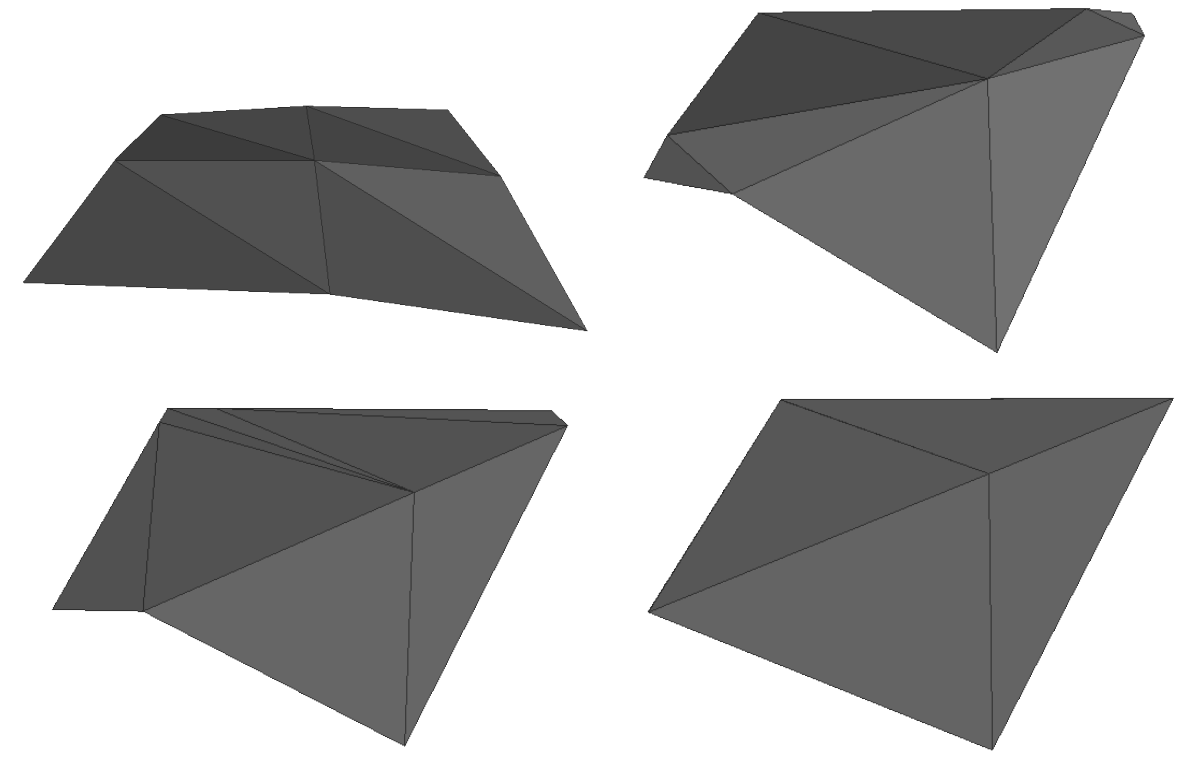

Figure 5.4.: First row: solutions for $\epsilon=1 / 2$ and $\epsilon=1 / 8$, with $k=10^{6}$; second row: solutions for $\epsilon=1 / 8$ and $\epsilon=1 / 1000$, with $k=10^{8}$.
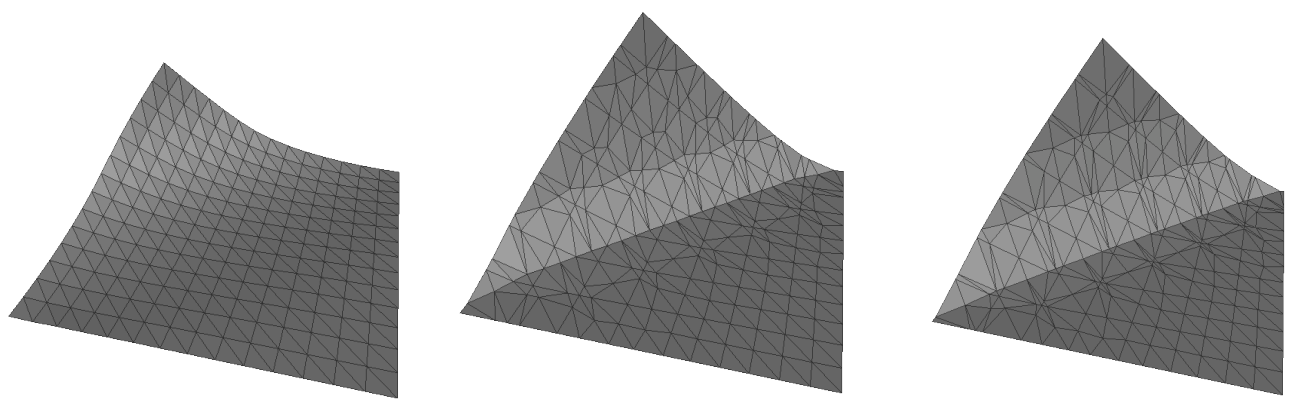

Figure 5.5.: From left to right: solutions for $k=10^{8}$ and $\epsilon=1 / 2,1 / 4,1 / 8$. Load is pointing upwards.

In our experiments, we consider a square plate fixed at three vertices with a point load applied to the fourth vertex. A smooth deformation is obtained for any finite stiffness $k$, as seen in the first two images in Fig. 5.4, while the nonsmooth case appears as load 
and stiffness blow up. The case $k=10^{8}$ is a good approximation of this limit, as seen in the other two images. As we increase the resolution, as shown in Fig. 5.5, edgepoints permit the formation of sharp rulings, which never clearly appear at any resolution for CST, indicated with $\epsilon=1 / 2$.

\section{Sensitivity to $k$ and $\epsilon$}

We here compare the solutions for different $k$ and $\epsilon$, at the coarsest level. The results can be seen in Fig. 5.6 and 5.7. From the former, we see that the value $\epsilon=1 / 4$ is enough to avoid rigidification, thanks to the formation of bending lines, which explains the gap observable between $\epsilon=1 / 2$ and $\epsilon=1 / 4$. Smaller values of $\epsilon$ only improve the placement of such lines, but the gain in terms of accuracy is less important. In all cases, a value of $\epsilon=1 / 8$ suffices to obtain a solution close to the case $\epsilon=0$.

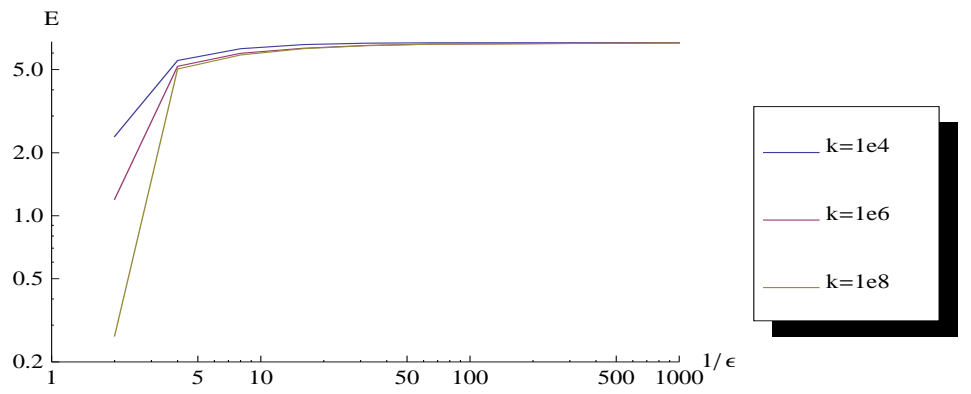

Figure 5.6.: Logarithmic plots of the energy value at the minimum, versus $\epsilon$, for different values of $k$.

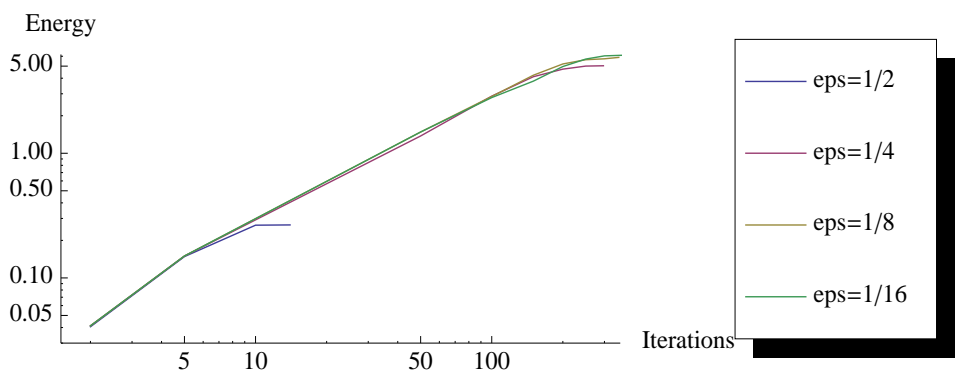

Figure 5.7.: Logarithmic plot of the energy value at the minimum, versus the Newton iterations, for $k=10^{8}$.

From Fig. 5.7, we see that the order of convergence of Newton's method does not degrade, since the slope of the four curves is independent of $\epsilon$. As expected, more iterations are required to compute a better minimizer, since the initial guess is progressively 


\section{Adaptive surfaces}

farther from the undeformed configuration. We thus have numerical evidence that, on this example, a finite value of $\epsilon$ does not affect the locking-free properties of the method and the order of convergence of Newton.

\section{Sensitivity to $h$}

We now perform a convergence analysis, using three different values of $k$ and uniform mesh refinement. The results can be seen in Fig. 5.8. The right column shows the convergence behavior in terms of the $L^{\infty}$ norm of the solution. From this measure, there is no sign of rigidification compared with a mesh having the edges aligned with the smooth rulings. However, the left column shows the convergence behavior in terms of the $L^{2}$ error and it becomes clear that the method is not optimal in terms of the shape approximation. In particular, the method performs very well on coarse meshes, but it exhibits the tendency to worsen as the resolution increases.

As can be seen in Fig. 5.5 and 5.9, this tendency can be due to the fact that each step of refinement does not double the number of rulings which can be approximated, since the number of edges which need to be traversed by each ruling grows with the number of vertices.
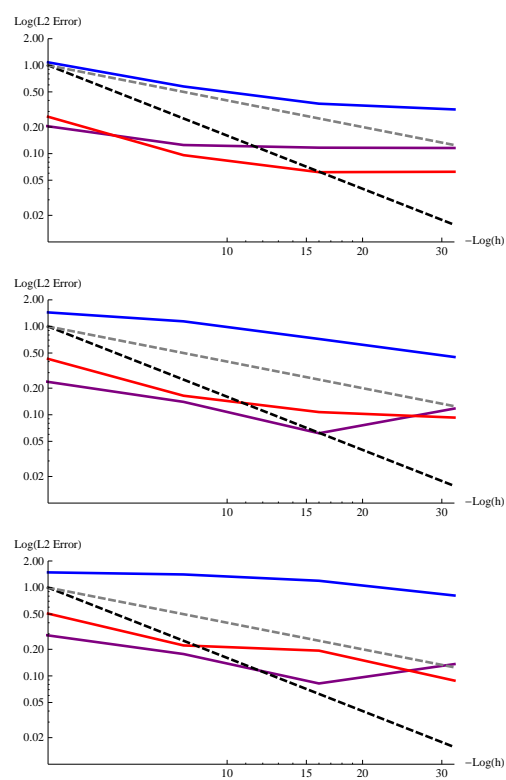
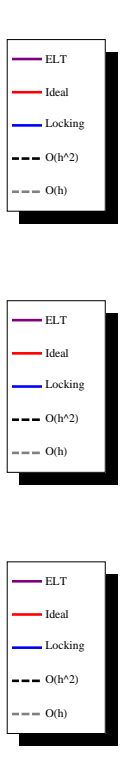
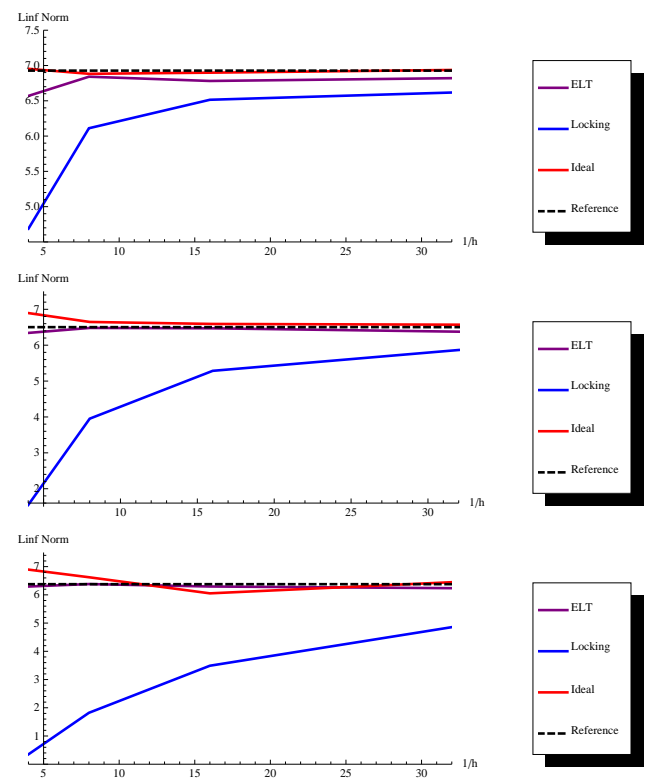

Figure 5.8.: Edgepoints. Left column: logarithmic plot of the $L^{2}$ error versus mesh resolution. Right column: $L^{\infty}$ norm versus mesh resolution. Each row corresponds to a different membrane stiffness $k=10^{2}, 10^{4}, 10^{6}$. We consider $h$ to be the mesh size after the subdivision. 


\section{Bifurcations}

We provide numerical evidence in Fig. 5.9 that there exist multiple stationary points of the energy, having significantly different shapes. From the pictures, it is evident that the different solutions can be characterized by having either one or two bending lines, which are formed depending on the position of some specific edgepoints, shown in red. The presence of bifurcation is troublesome for several reasons, e.g., we have no guarantee to reach a global minimum, together with a degradation of the order of convergence of Newton's method. Indeed, the optimal behavior shown in Fig. 5.7 is lost after refinement.
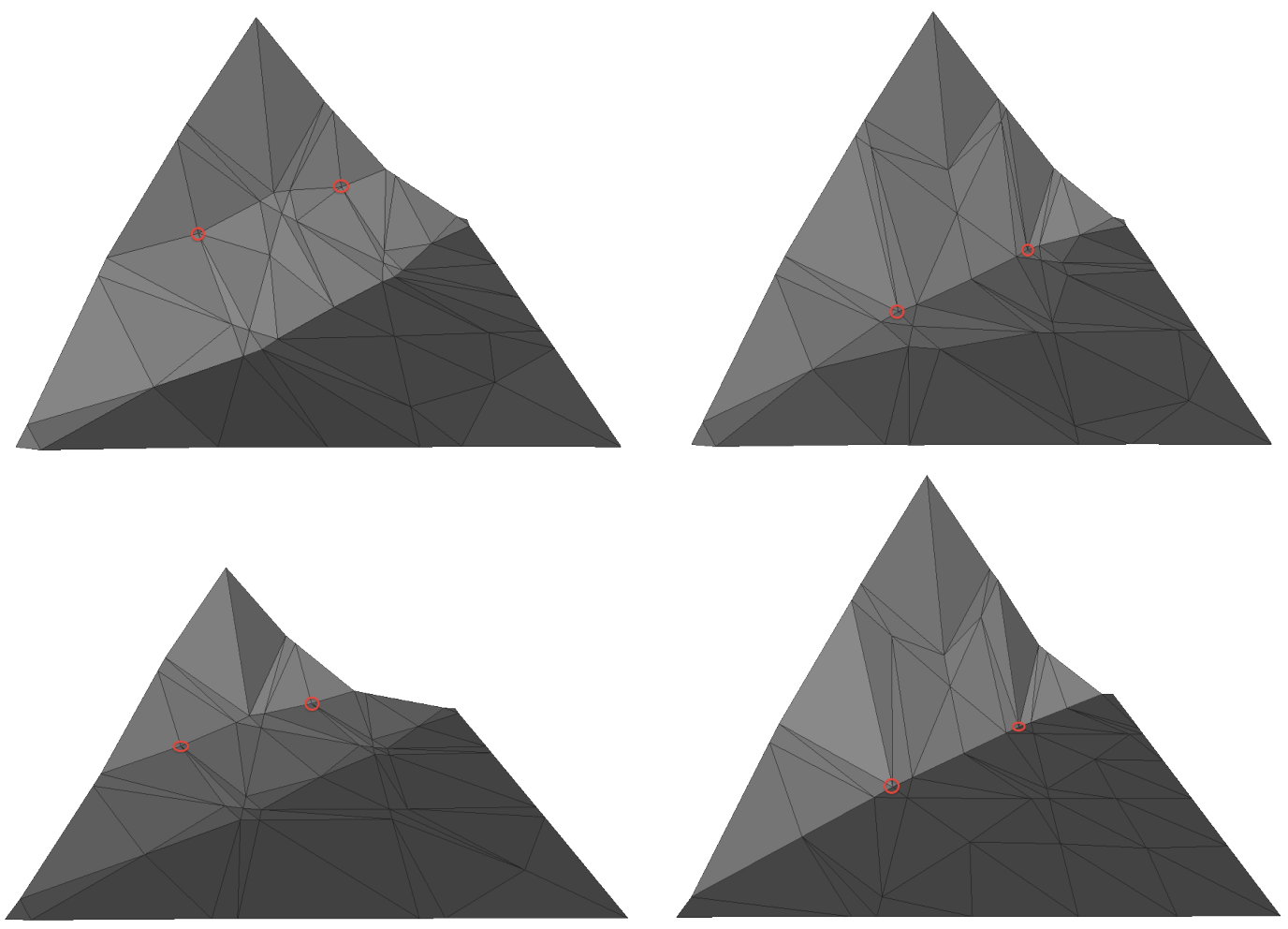

Figure 5.9.: Two pairs of different solutions for the same $\epsilon=1 / 8$ and $h$. The first row corresponds to $k=10^{6}$, the second row to $k=10^{8}$. Indicated in red are the edgepoints responsible for the formation of the main rulings.

\subsubsection{Counterexample}

A definitive argument against the use of edgepoints it the possibility to build a mesh which induces locking for any finite $\epsilon>0$, despite the use edgepoints, as sketched in Fig. 5.10 . Consider a regular mesh consisting of $M$ lines, having $N$ vertices each, equally spaced at distance $h$. Now, the barrier (5.3) induces a minimum distance $\epsilon>0$ at which 


\section{Adaptive surfaces}

we can place the edgepoints in proximity of the vertices. If we offset each line of $\epsilon$ with respect to its neighbor above, it follows that, when $\epsilon M>h$, the entire mesh is covered by the forbidden interval, thus it is rigid.

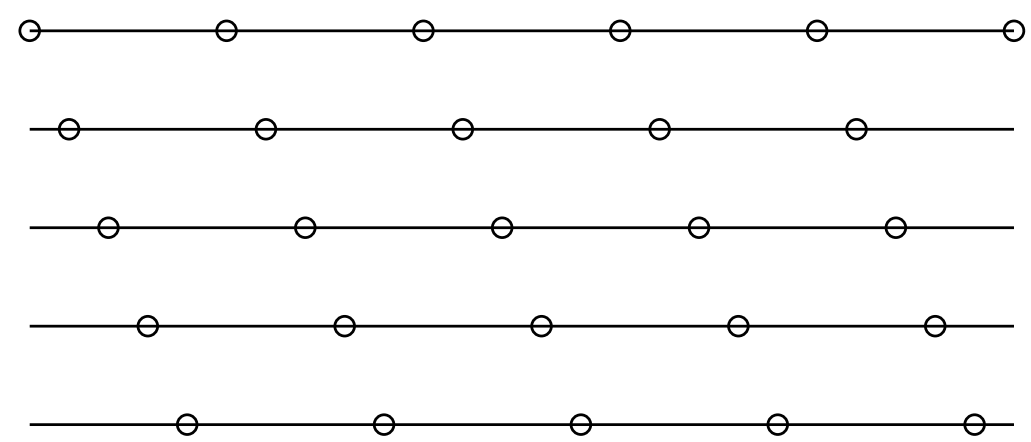

Figure 5.10.: Counterexample showing edgepoints rigidification. In this case we have 5 lines, so the forbidden interval must be of length at least $h / 5$ to cause rigidification, where $h$ is the mesh spacing.

\subsection{R-adaptivity}

In the previous section, we have presented a novel discrete model obtained from physical and geometrical considerations. At a closer inspection, this model belongs to a larger family of adaptive methods, called r-adaptivity. In particular, our special assumption that constrains the edgepoints to move in $1 \mathrm{D}$ along edges is equivalent to the r-adaptive treatment of the boundary of 3D bodies proposed by [53] and questioned by [35].

In order to avoid the counterexample presented in Fig. 5.10, we now consider a more general formulation of r-adaptivity for plates, in which all the nodes can move in the undeformed configuration, not just edgepoints, under the following restrictions: (i) interior nodes can move anywhere and (ii) boundary points can slide along straight boundaries, but they are fixed on curved boundaries.

\section{Motivations}

Let $W(\phi)$ be the potential energy associated to the deformation $\phi \in X$. In a discrete setting $X_{h} \subset X$, we seek to minimize the discrete version of the total potential energy as a functional of the discretized spatial deformation map

$$
W_{h}:=W\left(\phi_{h}\right)
$$

If $X_{h}$ is a Sobolev space, standard error estimates provide energy-norm errors $\left\|\phi_{h}-\phi\right\|_{W}$, provided that the exact solution $u$ has sufficient regularity, therefore a natural adaption strategy is to optimize the mesh to minimize this bound. 
For linear problems, generally $W(\phi)$ is convex and it is possible to show that the solution exists and it is unique. For linearized problems, the solution might not be regular, or convexity can be lost. For nonlinear problems, no solution might exists and no natural norm can be defined on $V$, so the above framework collapses. Therefore, we define a new measure for the error of a solution.

Definition 5.1. (Error measure) Let

$$
\begin{aligned}
d\left(\phi_{1}, \phi_{2}\right) & :=\left|W\left(\phi_{1}\right)-W\left(\phi_{2}\right)\right|, \quad \forall \phi_{1}, \phi_{2} \in X, \\
\phi_{\min } & :=\{\arg \min W(\phi)\} .
\end{aligned}
$$

We define the error measure of a function to be

$$
e\left(\phi_{h}\right):=d\left(\phi_{h}, \phi_{\min }\right) .
$$

Remark. For linear problems, the error $e$ reduces to the usual energy norm

$$
e\left(\phi_{h}\right)=\left|W\left(\phi_{h}\right)-W\left(\phi_{\min }\right)\right|=\left|\left\|\phi_{h}\right\|_{W}^{2}-\|\phi\|_{W}^{2}\right|=\left\|\phi_{h}-\phi\right\|_{W}^{2},
$$

where we made use of the orthogonality $\left\langle\phi_{h}-\phi, \phi_{h}\right\rangle_{W}=0$.

In order to be a meaningful measure, we must show that $d$ is a distance, that is we have to show that it is non-negative and positive-definite. Since $d \geq 0$ is trivial, we discuss the latter property in the following proposition.

Proposition 5.3. (Positive-definiteness) Let $\phi_{\min }$ and $d$ be defined as in (5.15) and (5.16). Then

$$
d\left(\phi_{h}, \phi_{\min }\right)=0 \Longleftrightarrow \phi_{h}=\phi_{\min }, \quad \forall \phi_{h} \in X_{h} \subset X .
$$

Proof. Let $\phi^{\prime} \in X_{h}$ be such that $\phi^{\prime} \neq \phi_{\min }$ and $d\left(\phi^{\prime}, \phi_{\min }\right)=0$. Since it holds also $\phi^{\prime} \in X$, by the previous preposition it follows that $\phi^{\prime}=\phi_{\min }$.

From the previous proposition, we conclude that $e$ is a distance and a meaningful error measure. Since $W\left(\phi_{\min }\right)$ is fixed, the best discretization $X_{h} \subset X$, is the one that minimizes $W\left(\phi_{h}\right)$. The idea of r-adaptivity is to notice that

$$
W=W(\phi, \bar{\phi}),
$$

where $\bar{\phi}$ defines the undeformed configuration, so the minimization should be performed in terms of both arguments of the energy.

\section{Shells}

In the case of initially straight developable surfaces, there are infinite meshes equally able to approximate the undeformed geometry. Unfortunately, in the case of shells, there are two major issues: 


\section{Adaptive surfaces}

- $\bar{\phi}$ must be constrained to represent the undeformed surface but, in general, the parametrization of the undeformed configuration is not given explicitly.

- Minimizing directly $W$ can significantly alter the approximation quality of the undeformed configuration since, in contrast to the interior of $3 \mathrm{D}$ bodies, the interior of a thin shell is void.

Assuming the parametrization to be given, a possible strategy to fix the second issue is to insert in the energy an additional term measuring the approximation of the undeformed curvature. We do not pursue this cumbersome strategy, since it is outside the scope of this work, but we here analyze the developable case.

\subsubsection{Kinematics}

Within the isoparametric concept, the discretized element-wise spatial deformation map $\phi_{h}^{e}$ and material placement $\bar{\phi}_{h}^{e}$ are given in terms of scalar-valued mappings, called the shape functions, $N_{k}(\xi)$ from the isoparametric coordinates $\xi \in[-1,+1]^{d}$ and nodal values $\phi_{k}$ and $\bar{\phi}_{k}$, respectively, with local node numbering $k$, see Fig. 12.

$$
\begin{aligned}
\bar{\phi}_{h}^{e} & =\sum_{k} N_{k}(\xi) \bar{\phi}_{k} \\
\phi_{h}^{e} & =\sum_{k} N_{k}(\xi) \phi_{k} .
\end{aligned}
$$

Accordingly, we may regard the discrete version of the total potential energy as a function of the collection of nodal values $\phi_{K}=\left\{\phi_{k}\right\}$ and $\bar{\phi}_{K}=\left\{\bar{\phi}_{k}\right\}$, respectively, with global node numbering $K$

$$
W_{h}:=W\left(\phi_{K}, \bar{\phi}_{K}\right) \text {. }
$$

Thus the minimization of (5.14) or (5.23) amounts to the search for the global minimum in $\phi_{K}$ and $\bar{\phi}_{K}$

$$
W\left(\phi_{K}, \bar{\phi}_{K}\right) \rightarrow \min .
$$

By allowing variations in both $\phi_{K}$ and $\bar{\phi}_{K}$, we obtain spatial and material residuals

$$
\begin{aligned}
r_{k} & :=\frac{\partial W_{h}}{\partial \phi_{h}}, \\
R_{k} & :=\frac{\partial W_{h}}{\partial \bar{\phi}_{h}} .
\end{aligned}
$$

These residuals turn out to be expressed in terms of the Cauchy stress $\sigma$ and the spatial volume force density $b^{t}$ for the spatial residuum and the Eshelby stress $\Sigma$ and the material volume force density $B_{0}$ for the material residuum

$$
\begin{aligned}
r_{K} & =\int_{\mathbb{B}_{h}^{t}} \sigma_{h} \cdot \nabla_{x} N_{K}-N_{K} b_{h}^{t} d V, \\
R_{K} & =\int_{\mathbb{B}_{h}^{0}} \Sigma_{h} \cdot \nabla_{\bar{x}} N_{K}-N_{K} B_{h}^{0} d V,
\end{aligned}
$$


where the first equation is the canonical force equilibrium. This implies that, for a given mesh, the second equation is in general not satisfied and therefore the discrete solution of a non-adaptive method is not optimal, leading to locking in the worst case scenario. For a more detailed presentation of r-adaptivity, see [46] and [57].

\subsubsection{Energy}

In the case of r-adaptivity, there is no limitation in choosing the energy contributions. We decided to employ CST and $\mathrm{CCH}$ for the membrane and bending contributions, whose novel computation of gradients and Hessians, with respect to the undeformed configuration, is presented in the appendices. Unfortunately, even for such simple energies, we found that the amount of terms to be computed slows down the assembly of the Hessians of a factor of 5 , when compared to the non-adaptive case.

\section{Stabilizer}

In the case of r-adaptivity, it is not trivial to avoid degenerate triangles by inserting a condition such as (5.3) inside the kinematic assumptions, so the energy is modified by introducing a new potential [46]

$$
W_{+}:=W+\alpha S(J)
$$

where $\alpha \in \Re$ and

$$
S(J) \rightarrow \infty \text { for } \quad J \rightarrow 0 .
$$

In other words, we need to build $S$ such that it blows up when a triangle collapses. Thus, a straightforward choice is to measure the area of the triangles as

$$
\begin{aligned}
& J=A, \\
& S=-\log (J),
\end{aligned}
$$

where $A$ is the area of the adapted configuration. However, the above energy can be improved in at least two ways

- In the non-adapted undeformed configuration, it does not hold $S=0$.

- There is no control on the quality of the triangles.

Therefore, [46] proposed to employ a neo-Hookean stabilization, which can be written using the first fundamental form as

$$
\begin{aligned}
& J=\operatorname{det}(I), \\
& S=\left(\frac{\operatorname{tr}(I)}{J^{2 / 3}}-2-2 \log (J)\right)+(J-1)^{2} .
\end{aligned}
$$

In the undeformed configuration, $J=1$ and $\operatorname{tr}(I)=2$, from which it follows $S=0$. Moreover, $S \rightarrow \infty$ as $J \rightarrow 0$, which is the case if $A \rightarrow 0$. 


\section{Adaptive surfaces}

\subsubsection{Numerical experiments}

We now proceed with a numerical validation of the method. The purpose of the tests is to investigate the robustness of r-adaptivity towards membrane locking, so we use again the simply supported plate example presented earlier, which represents the worst case scenario since the triangulation has no edge close to the rulings of the smooth solution.
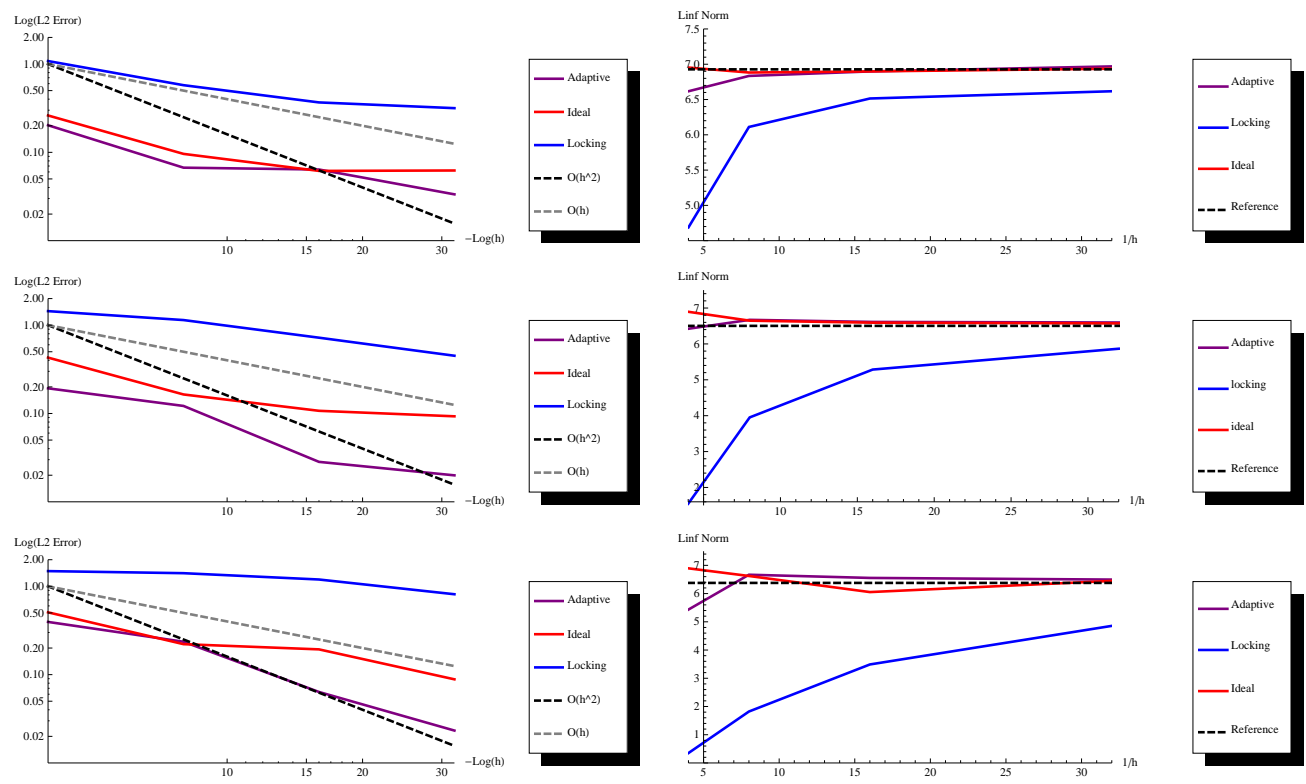

Figure 5.11.: Constant Curvature Hinge. Left column: logarithmic plot of the $L^{2}$ error versus mesh resolution. Right column: $L^{\infty}$ norm versus mesh resolution. Each row corresponds to a different membrane stiffness $k=10^{2}, 10^{4}, 10^{6}$.

We compared the results using $\mathrm{CCH}$ and MSO, whose results can be seen in Fig. 5.11 and 5.12, respectively. In the right column of both figures, we have plotted the $L^{\infty}$ norm of the solution, represented by the z-displacement at the loaded tip, which clearly indicates that, for both methods, rigidification is not present since the r-adaptive method behaves as the ideal triangulation, having the edges aligned with the rulings.

From the left column, we see a very interesting result: for low membrane stiffness, nonadaptive MSO is clearly superior to CS; however, as the stiffness increases, the accuracy of the two non-adaptive methods becomes very similar. Instead, when r-adaptivity is employed, the advantage of MSO even at finer resolutions becomes again evident. This, together with the absence of rigidification, suggests that the proposed method has optimal accuracy.

While accuracy is optimal, the same cannot be said for the robustness toward the stabilization parameter $\alpha$ in (5.29). Moreover, the Hessians are much denser than the non-adaptive case, their computation is up to five times slower, and they are singular or close to being singular, depending on the stabilization. Indeed, we found that increasing 

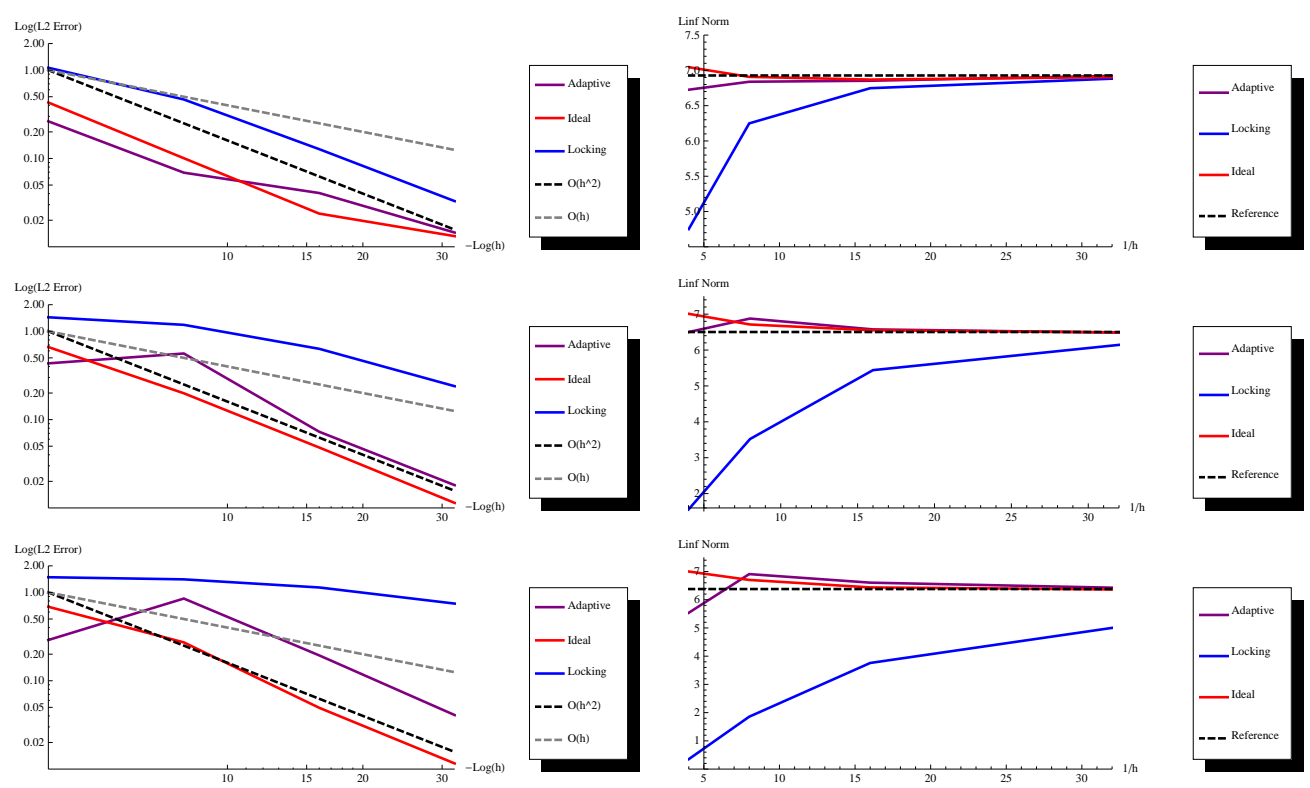

Figure 5.12.: Midedge Shape Operator. Left column: logarithmic plot of the $L^{2}$ error versus mesh resolution. Right column: $L^{\infty}$ norm versus mesh resolution. Each row corresponds to a different membrane stiffness $k=10^{2}, 10^{4}, 10^{6}$.

$\alpha$ improves the numerical efficiency of the method, but it can lead to locking or mesh dependence. These issues are further explored in the next section.

\subsection{Dynamics}

Unfortunately, the treatment of dynamic simulation in the context of r-adaptivity is particularly challenging for several reasons:

- The issue of bifurcations is particularly severe, since dynamic solvers tend to prefer a non-optimal solution close to the current configuration, rather than exploring large dislocations of the undeformed positions of the nodes, as done by energy minimization solvers.

- The kinetic energy is position-dependent, which yields a difficult derivation of the Euler-Lagrange equations and undermines the efficiency, since techniques such as mass-lumping do not lead to a constant or diagonal mass matrix.

- A naïve definition of the velocity field as the time derivative of the deformation, introduces instabilities in the system [57]. Therefore, it is necessary to use a mixed formulation where the velocity is interpolated as an independent variable, thereby further complicating the equations and affecting the efficiency of the computations. 


\section{Adaptive surfaces}

The above points are presented in more detail below, in particular we will show how to write the Euler-Lagrange equations using a generalized notion of the mass matrix for the non-adaptive and the adaptive case, including the case of an independent velocity field. We conclude the section by showing on a numerical example that, even when using such formulations and a fine mesh, the solution can exhibit a strong mesh dependence.

\subsubsection{Mass matrix}

We now compute the quantities corresponding to an extension of the usual mass matrix, which can be directly used into the variational time stepping scheme proposed by [57]. To use their notations, consider an undeformed domain $\Omega$ with coordinates $X \in \Re^{2}$, and a map $\phi: \Omega \subset \Re^{2} \rightarrow \Re^{3}$ such that the deformed configuration can be expressed as $x=\phi(X, t)$. If we assume $\phi$ to be piecewise linear, then:

$$
\phi(X, t)=\sum_{f \in F} \phi^{f}(X, t)=\sum_{f \in F} \sum_{i=1}^{3} x_{i}^{f} N_{i}^{f}(X, t)=\sum_{i \in V} x_{i} N_{i}(X, t),
$$

where the basis function $N_{i}^{f}(X, t)$ can be defined using barycentric coordinates:

$$
N_{i}^{f}(X, t)=\frac{\left(X_{j}^{f}-X_{k}^{f}\right) J\left(X-X_{k}^{f}\right)}{\left(X_{j}^{f}-X_{k}^{f}\right) J\left(X_{i}^{f}-X_{k}^{f}\right)}=\frac{e_{i}^{f} J\left(X-X_{k}^{f}\right)}{2 A^{f}}=\frac{e_{i}^{f} J\left(X-m_{i}\right)}{2 A^{f}},
$$

where $(i, j, k)$ is a circular permutation of $(1,2,3)$, and:

$$
J=\left(\begin{array}{cc}
0 & -1 \\
1 & 0
\end{array}\right), \quad m_{i}^{f}=\frac{X_{j}^{f}+X_{k}^{f}}{2}, \quad e_{i}^{f}=X_{k}^{f}-X_{j}^{f},
$$

where we have used that $e_{i}^{f} J\left(X_{k}^{f}-m_{i}^{f}\right)=-e_{i}^{f} J e_{i}^{f} / 2=0$. Then, the velocity field is:

$$
\dot{\phi}^{f}(X, t)=\sum_{i=1}^{3}\left(\dot{x}_{i}^{f} N_{i}^{f}(X, t)+x_{i}^{f} \dot{N}_{i}^{f}(X, t)\right) \text {. }
$$

The mass matrix is defined as the Hessian of the $L^{2}$ norm of the velocity field. For convenience, we split its computation into three $3 \times 3$ submatrices:

$$
\begin{aligned}
m_{i j}(X, t) & =\frac{1}{2} \frac{\partial^{2}}{\partial \dot{x}_{i} \partial \dot{x}_{j}} \int_{\Omega} \dot{\phi}(X, t)^{2}, \\
M_{i j}(X, t) & =\frac{1}{2} \frac{\partial^{2}}{\partial \dot{X}_{i} \partial \dot{X}_{j}} \int_{\Omega} \dot{\phi}(X, t)^{2}, \\
\tilde{M}_{i j}(X, t) & =\frac{1}{2} \frac{\partial^{2}}{\partial \dot{x}_{i} \partial \dot{X}_{j}} \int_{\Omega} \dot{\phi}(X, t)^{2} .
\end{aligned}
$$




\section{Non-adaptive surface}

Since $\dot{N}_{i}^{f}(X, t)=0$ for the non-adaptive case, we have:

$$
\dot{\phi}(X, t)^{2}=\left(\sum_{f \in F} \dot{\phi}^{f}\right)^{2}=\sum_{f \in F} \sum_{a=1}^{3} \sum_{b=1}^{3}\left(\dot{x}_{a}^{f} \cdot \dot{x}_{b}^{f}\right) N_{a}^{f} N_{b}^{f} \approx \sum_{f \in F} \sum_{a=1}^{3}\left(x_{a}^{f}\right)^{2} \sum_{b=1}^{3} N_{a}^{f} N_{b}^{f},
$$

where we have used the mass lumping assumption. It follows that $M_{i j}=\tilde{M}_{i j}=0$ and the remaining block reduces to the canonical lumped mass matrix

$$
m_{i j}=I d_{3} \sum_{f \in F} \sum_{a=1}^{3} \delta_{a i} \delta_{a j} \sum_{b=1}^{3} \int_{\Omega} N_{a}^{f} N_{b}^{f}=I d_{3} \sum_{f \in F} \sum_{a=1}^{3} \delta_{a i} \delta_{a j} \frac{1}{3} A^{f},
$$

where $I d_{3}$ is the $3 \times 3$ identity matrix.

\section{Adaptive surface}

For the adaptive case, we need to compute the time-derivative of the basis function using the chain-rule:

$$
\dot{N}_{i}^{f}(X)=-\frac{\partial N_{i}^{f}}{\partial X} \cdot \dot{X}=-\frac{\partial N_{i}^{f}}{\partial X} \cdot \sum_{a=1}^{3} N_{a}^{f} \dot{X}_{a}^{f}=-\frac{e_{i}^{f} J}{2 A^{f}} \cdot \sum_{a=1}^{3} N_{a}^{f} \dot{X}_{a}^{f}=t_{i}^{f} \cdot \sum_{a=1}^{3} N_{a}^{f} \dot{X}_{a}^{f} .
$$

It follows that the velocity field is

$$
\dot{\phi}^{f}(X)=\sum_{a=1}^{3}\left(\dot{x}_{a}^{f} N_{a}^{f}+x_{a}^{f} t_{a}^{f} \cdot \sum_{b=1}^{3} N_{b}^{f} \dot{X}_{b}^{f}\right)=\sum_{a=1}^{3}\left(N_{a}^{f} \dot{x}_{a}^{f}+N_{a}^{f} \dot{X}_{a}^{f} \cdot \sum_{b=1}^{3} x_{b}^{f} t_{b}^{f}\right),
$$

and its $L^{2}$ norm is

$$
\|\dot{\phi}(X)\|^{2}=\sum_{f \in F}\left\|\dot{\phi}^{f}(X)\right\|^{2}
$$

where the norm on a single face can be approximated using mass lumping

$$
\begin{aligned}
& \sum_{a, b=1}^{3} N_{a}^{f} N_{b}^{f}\left(\dot{x}_{a}^{f} \cdot \dot{x}_{b}^{f}+\sum_{c, d=1}^{3}\left(\dot{X}_{a}^{f} \cdot t_{c}^{f}\right)\left(\dot{X}_{b}^{f} \cdot t_{d}^{f}\right)\left(x_{c}^{f} \cdot x_{d}^{f}\right)+2 \sum_{c=1}^{3}\left(\dot{X}_{a}^{f} \cdot t_{c}^{f}\right)\left(x_{c}^{f} \cdot \dot{x}_{b}^{f}\right)\right) \approx \\
& \sum_{a=1}^{3}\left(\left\|\dot{x}_{a}^{f}\right\|^{2}+\sum_{c, d=1}^{3}\left(\dot{X}_{a}^{f} \cdot t_{c}^{f}\right)\left(\dot{X}_{a}^{f} \cdot t_{d}^{f}\right)\left(x_{c}^{f} \cdot x_{d}^{f}\right)+2 \sum_{c=1}^{3}\left(\dot{X}_{a}^{f} \cdot t_{c}^{f}\right)\left(x_{c}^{f} \cdot \dot{x}_{a}^{f}\right)\right) \sum_{b=1}^{3} N_{a}^{f} N_{b}^{f} .
\end{aligned}
$$




\section{Adaptive surfaces}

By using that $\sum_{b=1}^{3} \int_{f} N_{a}^{f} N_{b}^{f}=\frac{1}{3} A^{f}$, it follows that the blocks of the mass matrix are

$$
\begin{aligned}
m_{i j} & =I d_{3} \sum_{f \in F} \sum_{a=1}^{3} \delta_{a i} \delta_{a j} \frac{1}{3} A^{f} \\
M_{i j} & =\sum_{f \in F} \sum_{a=1}^{3} \delta_{i a} \delta_{j a}\left(\sum_{c=1}^{3} \sum_{d=1}^{3} t_{c}^{f} \otimes t_{d}^{f}\left(x_{c}^{f} \cdot x_{d}^{f}\right)\right) \frac{1}{3} A^{f}, \\
\tilde{M}_{i j} & =\sum_{f \in F} \sum_{a=1}^{3} \delta_{i a} \delta_{j a} \sum_{c=1}^{3}\left(t_{c}^{f} \otimes x_{c}^{f}\right) \frac{1}{3} A^{f},
\end{aligned}
$$

where $\otimes$ denotes the dyadic product of two vectors. We immediately notice that in the adaptive case, mass lumping generates a non-diagonal, non-constant, and possibly singular mass matrix

\section{Mixed Hamilton Principle}

As explained in [57], the time derivative $\dot{\phi}$ is only $L^{2}$ and this lack of regularity can cause a propagation of instabilities into the system, Therefore, we need to introduce an additional variable representing a $H^{1}$ velocity field

$$
V(X, t)=\sum_{f \in F} V^{f}(X, t)=\sum_{f \in F} \sum_{i=1}^{3} V_{i}^{f} N_{i}^{f}(X, t)=\sum_{i \in V} V_{i} N_{i}(X, t),
$$

from which the kinetic energy is trivially computed using linear elements:

$$
\frac{1}{2} \int_{\Omega} V^{2}=\frac{1}{2} V^{T} m(X, t) V .
$$

In this case, $\tilde{M}(X, t)$ does not appear in the equations, while $M(X, t)$ simplifies the computation of the following the mixed product:

$$
\begin{aligned}
\int_{\Omega} V \cdot \dot{\phi} & =\int_{\Omega} \sum_{f \in F} \sum_{a=1}^{3} \sum_{b=1}^{3} N_{a}^{f} N_{b}^{f}\left(\dot{x}_{a}^{f} \cdot V_{b}^{f}+\sum_{c=1}^{3}\left(\dot{X}_{a}^{f} \cdot t_{c}^{f}\right)\left(x_{c}^{f} \cdot V_{b}^{f}\right)\right) \\
& \approx \int_{\Omega} \sum_{f \in F} \sum_{a=1}^{3}\left(\dot{x}_{a}^{f} \cdot V_{a}^{f}+\sum_{c=1}^{3}\left(\dot{X}_{a}^{f} \cdot t_{c}^{f}\right)\left(x_{c}^{f} \cdot V_{a}^{f}\right)\right) \sum_{b=1}^{3} N_{a}^{f} N_{b}^{f} \\
& =V^{T} m(X, t) \dot{x}+V^{T} M(X, t) \dot{X} .
\end{aligned}
$$

Altogether, the additional term arising in the mixed Hamilton principle presented in [57] is obtained in a compact form:

$$
\int_{\Omega} P \cdot(\dot{\phi}-V)=\int_{\Omega} V \cdot(\dot{\phi}-V)=V^{T}(m(X, t) \dot{x}+M(X, t) \dot{X}-m(X, t) V),
$$

where $P$ is the linear momentum. Again, the global mass matrix formed by the blocks $m(X, t)$ and $M(X, t)$ is non-diagonal, non-constant, and possibly singular. 


\subsubsection{Numerical experiments}

We here perform two tests using the mass matrix computed above. The first tests compares this matrix with the canonical one, showing the necessity to use the former, while the second test illustrates the difficulty in obtaining a mesh-independent solution.

\section{Supported plate}

We consider the dynamic version of the usual simply supported plate we have employed to far. As can be seen from the frames captured at fixed interval of times in Fig. 5.13 and 5.14, the use of a standard mass matrix does not forbid the formation of a ruling, but slows down its formation, causing severe visual artifacts. On the contrary, the radaptive mass matrix is singular, and therefore it allows for instantaneous adaptation of the nodes in the undeformed configuration.
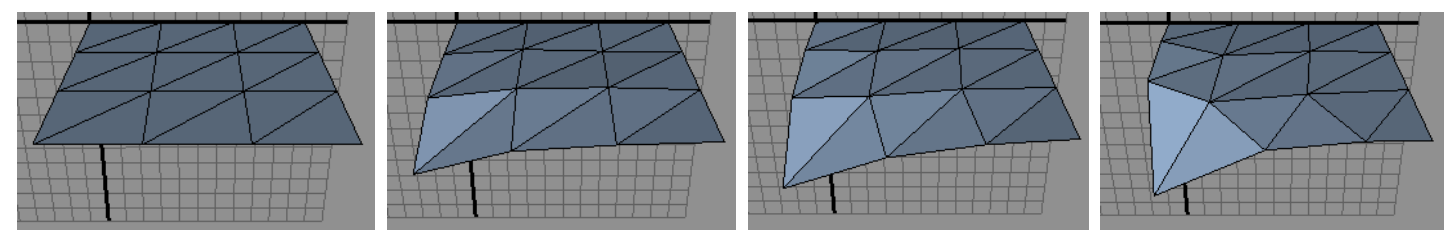

Figure 5.13.: Dynamical simulation of a supported plate with a standard constant mass matrix. Frames are captured every 40 fixed time steps.
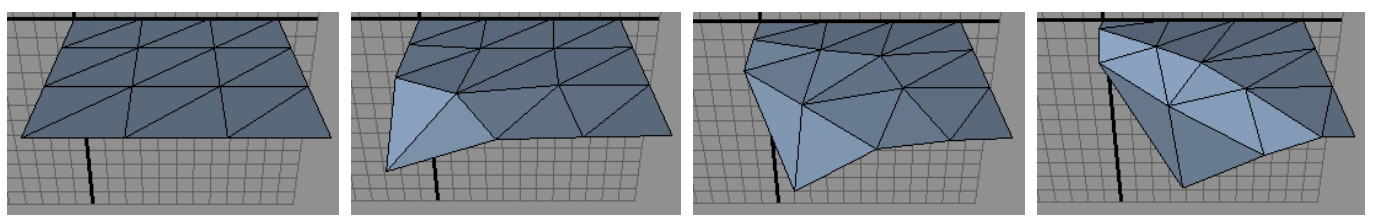

Figure 5.14.: Dynamical simulation of a supported plate with the r-adaptivity mass matrix. Frames are captured every 40 fixed time steps

\section{Draping}

In order to show the difficulties in obtaining a mesh-independent solution, we used two meshes with regular and irregular patterns, as shown in Fig. 5.15. As clear from the figure, despite the high number of DOFs, the solution shows a very strong mesh dependence. In fact, for this example, we found that an isotropic mesh with no adaptivity performs better than an anisotropic one with adaptivity.

For the reasons presented in this section, we will abandon r-adaptivity and look for more geometry- and physics-inspired non-adaptive discrete membrane models in the following chapter. 

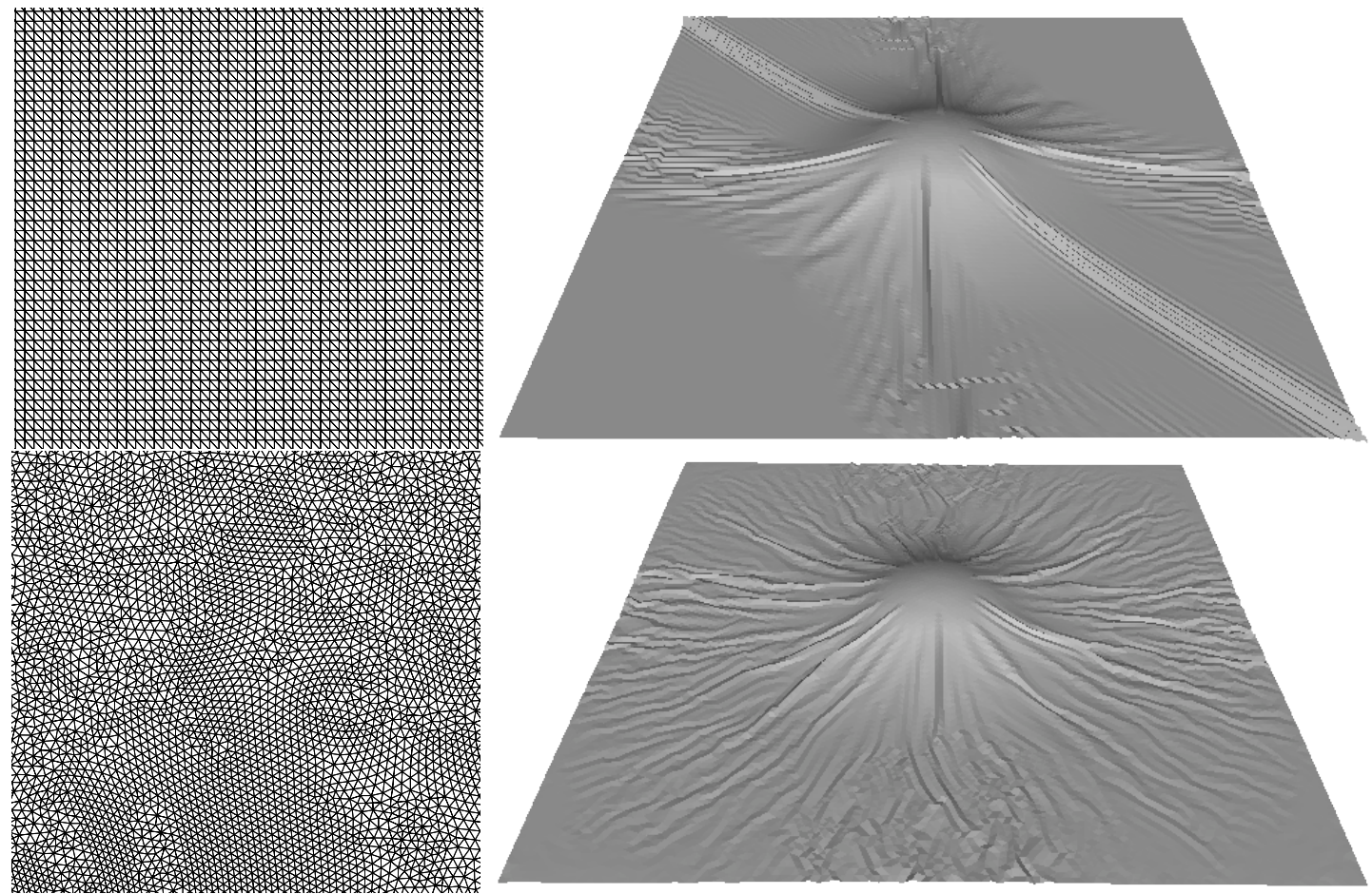

Figure 5.15.: Mesh dependence in draping over a sphere with r-adaptivity.

\subsection{Energy-based combinatorics}

So far, we have only considered fixed combinatorics. Despite not being interested in pursuing a detailed analysis of $h r$-adaptivity as found in [35], we point out peculiar properties of surfaces requiring a specialized treatment, in contrast to 3D elasticity.

In general, two strategies are used in combination with $r$-adaptivity: edge flips and h-adaptivity [35]. For reasons which will become clear in the following, we refer to these strategies as extrinsic and intrinsic flips, illustrated in Fig. 5.16 and 5.17, respectively.

A major difficulty with these strategies, especially for dynamical simulations, is the treatment of non-flat configurations since, e.g., energy jumps can be introduced. Despite these issues, the understanding of energy-based combinatorics will prove to be fundamental in the development of the next chapter.

\section{Extrinsic flips}

The idea behind this strategy is known under the name of edge swaps or Lawson flips, which is illustrated in Fig. 5.16. In short, the two triangles adjacent to each edge form a quadrilateral, which is split into a hinge by one of its diagonals. Since there are two possible ways to split a quadrilateral, both options are tested and the one with lower energy is preferred. The algorithm terminates when all the hinges are locally optimal. 

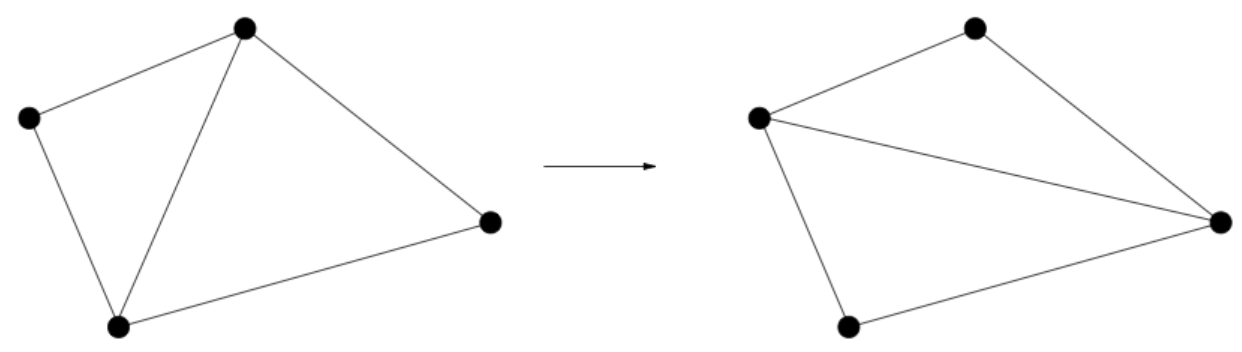

Figure 5.16.: Extrinsic flip of a hinge.

It is a known fact [35] that it is possible to attain any triangulation of a given set of nodes by a sequence of flips. However, there is no guarantee that the global minimum of the energy is attained, since the class of compositions of optimally local transformations does not contain all admissible triangulations [35]. Better algorithms can be devised, at the price of a higher computational cost.

In the case of surfaces, there is a clear situation in which the flip is energetically favorable, i.e., when the current diagonal is more compressed than the alternative one. In practice, we found that a minimal amount of bending in the hinge prevents any flip, since it induces a compression of the alternative diagonal which is significantly larger than the one present in the current diagonal, even in a rigidified situation when flipping could potentially lead to a formation of a global ruling.

\section{Intrinsic flips}

In this case, as shown in Fig. 5.17, a new node is introduced in the hinge if the local minimization of its position lowers the energy above a certain threshold. Clearly, this strategy solves the issue present with the extrinsic flips when the hinge is bent, since the new point can be placed such that the new diagonal is not straight and therefore not compressed.
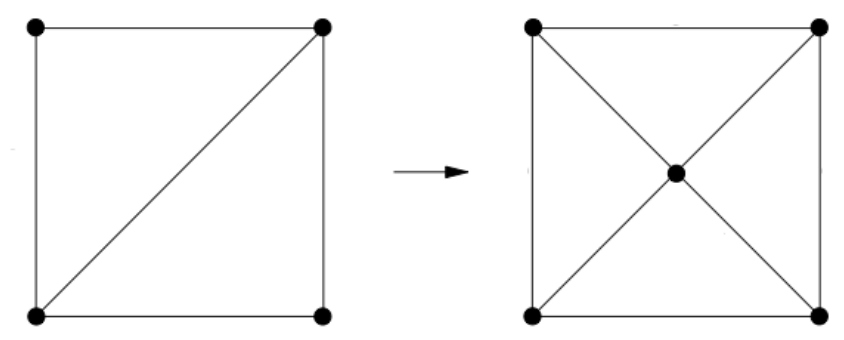

Figure 5.17.: Intrinsic flip of a hinge. 


\section{Adaptive surfaces}

In practice, we found that while performing better than extrinsic flips, this strategy is in general not sufficient to decrease the energy, since if a single hinge is not refined a ruling is broken and bending can be disallowed. Additionally, it is not clear how to define a coarsening strategy and, in the case of dynamical simulations, jumps in the forces cause severe visual artifacts.

Despite these issues, intrinsic flips posses an interesting physical interpretation, obtained by considering the membrane strains inside each triangle. Since the strain tensor is a symmetric $2 \times 2$ matrix, it has two real eigenvalues $\lambda_{1}, \lambda_{2} \in \Re$ and three cases are possible:

- Tension: $\lambda_{1}, \lambda_{2}>0$; the triangle is already in a nearly-optimal state, i.e., refinement would not significantly decrease the energy.

- Compression: $\lambda_{1}, \lambda_{2}<0$; the triangle might hide a cone singularity, i.e., refinement decreases the energy if buckling can be captured.

- Locking: $\lambda_{1} \lambda_{2}<0$; the triangle would like to bend, i.e., refinement could significantly reduce the energy.

Therefore, there is no need to perform a minimization for each newly inserted point, but the necessity of refinement can be directly inferred from the strain tensor. Interestingly, this discussion has lead us to a treatment of adaptivity which is very similar to the study of wrinkling models, which we look more closely at in the next chapter. 


\section{Mimetic surfaces}

In Chapter 3, we have presented the different asymptotic regimes that surfaces can exhibit, under given loads and boundary conditions. Here, we are interested in formulating discrete shell theories which preserve such asymptotic behaviors. The observation that plates prefer to buckle under uniaxial compression is not new, and it is known as wrinkling in the engineering community [34]. However, wrinkling models are employed to study unstable membrane deformations, such as the formation of wrinkles and folds in inflatable structures, but not bending-dominated regimes.

In this chapter, we propose a novel treatment of wrinkling, which makes possible its application to bending-dominated deformations. The main difficulty lies in the fact that known wrinkling theories neglect any bending contribution, motivated by the fact that inflatable structures are dominated by membrane stresses. On the contrary, we look for a discrete kinematic model that induces an increase of curvature, but not a change of metric, whenever compressive stresses are present.

We investigate and discuss several ideas to obtain a shell model mimicking the smooth regimes, which for this reason we call mimetic. We will proceed as follows:

- We start by understanding the physics and the importance of wrinkling, focusing in particular on devising a geometric description, leading to a novel discrete kinematic model which does not rigidify and does not lead to zero-energy modes.

- We continue by proposing a novel energetic formulation which yields to such kinematics in the inextensible limit. We also show that this framework generalizes the discrete shell theories presented in Chapter 4 and it can be interpreted as a geometrically nonlinear choice of mixed $\mathrm{FE}$ for $2 \mathrm{D}$ elasticity and plate problems.

- We conclude by providing numerical examples to support the validity of each component of our approach, testing separately membrane- and bending-dominated regimes, mesh dependence, and more complicated scenarios.

\subsection{Physics of locking}

In the end of Chapter 4, we have argued that the shortcomings of reduced integration techniques have a twofold origin: (i) not distinguishing between in-plane and normal displacements, and (ii) not differentiating between compressional and tensional stresses. This is often a source of misunderstandings, e.g., a membrane element such as CST, known to suffer from locking, is in fact better adequate for a membrane-dominated deformation than EAS or MITC methods, which are known to produce spurious modes or artificial softening in such a regime. 


\section{Mimetic surfaces}

In the previous chapter, we considered physically-based criteria for h-adaptivity. They are based on the analysis of the eigenvalues of the Cauchy-Green strain tensor and the insertion of new points along compressed edges. As we discussed, this strategy is inherently global and thus not robust, since the introduction of local rulings does not guarantee the avoidance of mesh rigidification.

Here, we present the approximation of the mixed behavior of inflatable structures, which are characterized by regions of membrane-dominated deformations, which look smooth, and regions of unstable membrane regimes where the material buckles, which are characterized by the presence of wrinkles, as can be seen in Fig. 6.1.

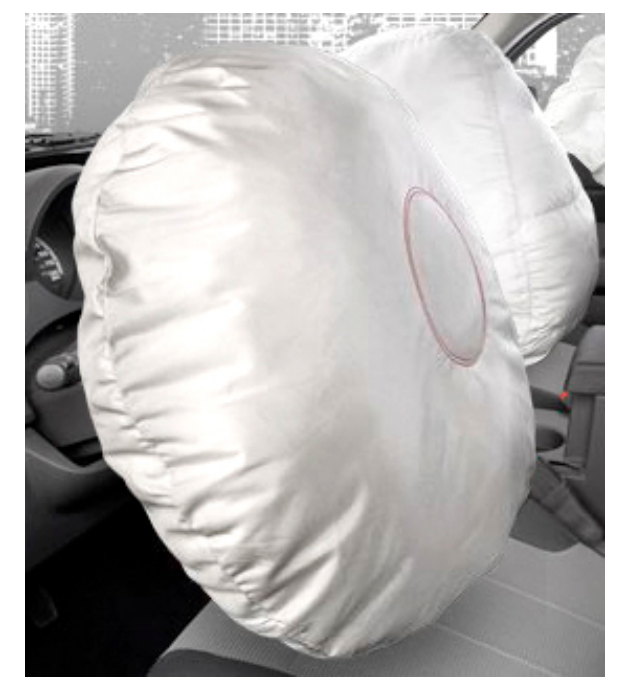

Figure 6.1.: Wrinkles in an inflated airbag.

In this approximation, called a wrinkling model, the bending contribution is completely neglected. Instead, our goal is to define a discrete kinematics that induces bending whenever a compressional stress state would cause buckling in the smooth theory. In fact, we argue that the issues encountered in the quest for shell FE arise when a discrete shell theory does not capture the correct smooth asymptotic behavior. In particular, we distinguish between two cases:

- The smooth regime is bending-dominated while the discrete one is membranedominated; membrane stresses are largely overestimated and locking occurs.

- The smooth regime is membrane-dominated while the discrete one is bendingdominated; membrane stresses are largely underestimated and failure modes occur.

The above offers a new perspective over the analysis performed in Chapter 4. Indeed, by working with traditional FE we always fall in one of the two categories, since we do not have direct control over the kinematics which results from a certain polynomial assumption. In our case, the kinematics is instead obtained by fulfilling certain given 
requirements and the difficulty is transferred to define a corresponding energetic term, as explained in the next sections.

\subsubsection{Wrinkling of membranes}

Before proceeding to the bending-dominated deformations, we must first understand the membrane-dominated case. In this scenario, locking is present when some areas of the body are subjected to uniaxial compressions, albeit it is not always recognized under this name, but is referred to as the inability to represent wrinkles [45]. In this case, locking appears since the mesh resolution cannot capture the small scale of the wrinkles. A well-known remedy, formulated as an energy-minimization problem by [34], is to define an augmented Cauchy-Green tensor

$$
\epsilon_{r}=\epsilon+a^{2} v \otimes v+b^{2} u \otimes u=\epsilon+\epsilon_{w}
$$

where $a, b \in \mathbb{R}$, the vectors $v, u \in \mathbb{R}^{3}$ span a cartesian coordinate system, and $\epsilon$ is the canonical Cauchy-Green tensor. In this case, $a, b$, and the orientation of the frame $u, v$ are free variables, which permit to find a better global minimizer of the elastic energy. The positivity of $a^{2}$ and $b^{2}$, implies that the minimum principle does not penalize compressions, i.e., it will produce a solution free of compressive stresses, whose overestimation is the culprit of locking.

As will become clear in the following, in the discrete setting there is no unique way to define wrinkling and our quest is to find an optimal choice. In the case of membranes, if the mesh is fine enough and the bending contribution is taken in account, then any FE for shells can be used and there is no need for employing wrinkling models [55].

\section{A multiscale view}

In order to acquire a better geometric and physical intuition of the asymptotic regimes, we propose a novel analogy with the motion of fluids. Other authors prefer instead to refer to plasticity theory in order to understand wrinkling [31].

In fluid dynamics, the equations for an incompressible and inviscid fluid on a domain $M$ describe a geodesic motion on $\operatorname{SDiff}(M)$, the group of smooth volume-preserving diffeomorphisms of $M \subset \mathcal{R}^{n}$ [41]. In other words, they arise from the constrained variation of a Lagrangian, which is the kinetic energy of the fluid. These equations can also be obtained as the limit of vanishing viscosity of Navier-Stokes equations, a case in which smooth solutions blow up and pointwise quantities lose their meaning [29].

Geometrically, the basic building block of a discrete representation of $\operatorname{Diff}(M)$ is represented by vorticity [41]. However, it is well known that is not feasible to approximate all the scales of vortices for a given Reynolds, thus the need for turbulence models. When viscosity increases, vortices of increasing size appear, and it is known that the accuracy in the approximation of the incompressibility constraint is a function of the smallest representable vortex for a given mesh [11].

In the case of thin structures, the equations for an isometric shell describe the constrained variation of the bending energy over the manifold represented by the shell itself, 


\section{Mimetic surfaces}

which can be described by a gradient field of $S O(3)$ frames. As the bending stiffness vanishes, a surface can develop an infinite number of cone singularities, meaning that the frames become uncoupled. As the bending stiffness increases, a coupling appears and the curvature becomes finite. Therefore, in the same way as for turbulence models, we argue that wrinkling models are needed in order to represent all the scales of curvatures on a coarse mesh.

\subsubsection{Two important obstacles}

As anticipated, it is not trivial to obtain a physically consistent kinematic model assuming that the deformation is given by a certain polynomial, as done in FE. Our approach is based instead on the definition of a set of minimal requirements which have to be satisfied at any coarse level. For this reason, our building block is represented by a hinge made of two triangles. In particular, we focus on two opposite scenarios, one demanding for enough flexibility in order to bend along arbitrary directions, the other one for enough rigidity to avoid in-plane deformations.

\section{Simply supported plate (SSP)}

The first requirement is important to validate the flexibility of the model. Consider a thin sheet having infinite membrane stiffness and represented as a mesh having four vertices and two triangles. Three vertices are fixed and a force in the out-of-plane direction is applied to the fourth vertex, resulting in the deformation represented in Fig. 6.2. The requirement is that for any choice of the three vertices, the displacement of the fourth vertex is nonzero. Otherwise the test is failed and we say that the kinematic model induces locking.

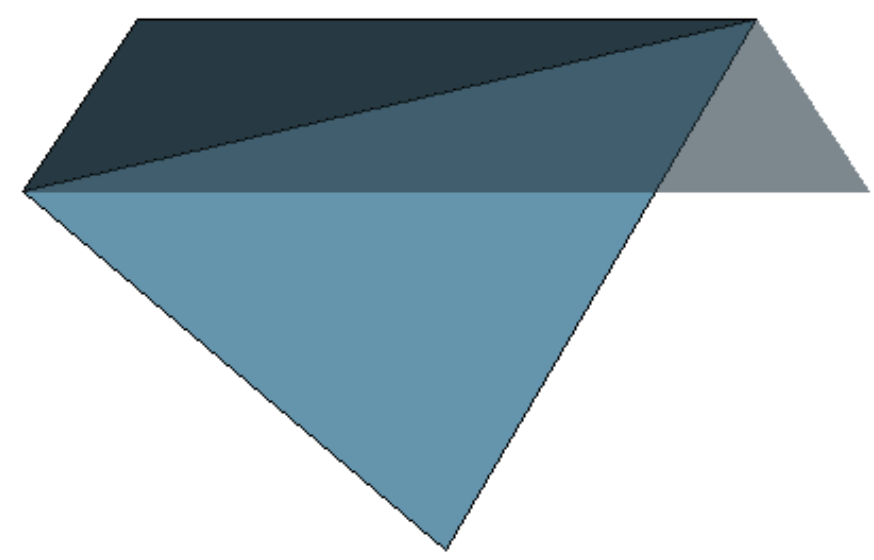

Figure 6.2.: Undeformed and deformed configurations for the simply supported plate. 


\section{Push-pull failure mode (PPM)}

The second requirement is important to validate the in-plane rigidity of the model. Consider a thin sheet having infinite membrane stiffness and represented as an hinge made of two triangles. A compressive force is applied along the inner edge and a traction along its orthogonal direction. The requirement is that there must exist no configuration in which the extrinsic distances between vertices increases. On the contrary, if the four vertices do not leave the plane and the extrinsic distance between them increases, as shown on the right of Fig. 6.3, then the test is failed.
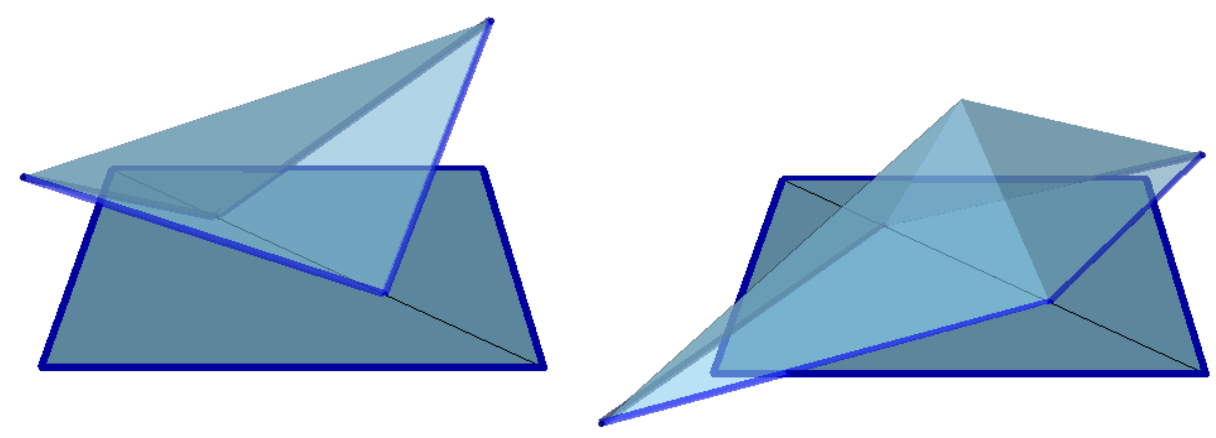

Figure 6.3.: Undeformed (flat), acceptable (left), and unacceptable (right) deformed configurations for the push-pull test. In both cases, the blue edges preserve their lengths.

We remark that, in the limit of vanishing thickness, the bending energy generated during wrinkling is negligible in a membrane dominated deformation. Therefore, the existence of some coupling between wrinkling and bending, which induces an increase curvature under compressions, is not enough to prevent the appearance of failure modes involving artificial stretching. In other words, the deformation on the right of Fig. 6.3 is not strictly a zero-energy mode, since it induces bending energy, but it is nevertheless a failure mode of the membrane model, since the amount of discrete energy generated in negligible compared to the corresponding smooth deformation.

\subsection{Kinematics}

As anticipated, it is not trivial to define a discrete notion of wrinkling. We here present several approaches tailored at building a kinematic model from first principles, each one focused at considering wrinkling of a different discrete entity. In this section, we will consider the problem of enforcing the isometry constraint $\epsilon=0$ with no locking or failure modes, without discussing how and if such constraint could arise as the limit $t \rightarrow 0$ of a stretching energy. We will address such question in the next section. 


\section{Mimetic surfaces}

\section{Wrinkling of triangles}

The most trivial application of wrinkling can be obtained by writing the CST energy in terms of the principal directions of the deformation

$$
\epsilon=\left(\begin{array}{cc}
\lambda_{1} & 0 \\
0 & \lambda_{2}
\end{array}\right)
$$

where $\lambda_{i}$ are the eigenvalues of the CST strain tensor $\epsilon$. The modified energy is built by allowing zero-energy compressions as

$$
\tilde{\epsilon}=\left(\begin{array}{cc}
\lambda_{1}+a_{1}^{2} & 0 \\
0 & \lambda_{2}+a_{2}^{2}
\end{array}\right),
$$

from which it follows that $\tilde{\epsilon}=0$ has a solution if $\lambda_{i} \leq 0$ for $i=1,2$.

Unfortunately, locking is still an issue for the SSP test. To see this, notice that in order to lift the free vertex, it is possible to compress the edges of the triangles, but not without increasing their heights, which is a non-zero energy mode for the energy 6.3.

\section{Wrinkling of edges}

A second application of wrinkling can be obtained by writing the strain as a 3-vector of constraints.

$$
\left(\begin{array}{l}
\epsilon\left(e_{1}\right) \\
\epsilon\left(e_{2}\right) \\
\epsilon\left(e_{3}\right)
\end{array}\right)=\left(\begin{array}{l}
\left\|e_{1}\right\|^{2}-\left\|\bar{e}_{1}\right\|^{2} \\
\left\|e_{2}\right\|^{2}-\left\|\bar{e}_{2}\right\|^{2} \\
\left\|e_{2}\right\|^{2}-\left\|\bar{e}_{3}\right\|^{2}
\end{array}\right)=\left(\begin{array}{c}
I_{1}-\bar{I}_{1} \\
I_{2}-\bar{I}_{2} \\
I_{3}-\bar{I}_{3}
\end{array}\right),
$$

where $I_{i}$ are the actions of the first fundamental form, pulled-back on the undeformed surface. The modified strain has in this case an additional parameter $a_{3}$

$$
\tilde{\epsilon}\left(e_{i}\right)=\left\|e_{i}\right\|^{2}-\left\|\bar{e}_{i}\right\|^{2}+a_{i}^{2}, \quad i=1,2,3 .
$$

Unfortunately, the test PPM is failed. To see this, notice that the above model only requires edges to compress, introducing no coupling inside triangles. Therefore, the triangle's height is allowed to stretch not only for bent configurations, as required to avoid locking, but also for membrane-dominated deformations, such as PPM.

\section{Wrinkling of polynomials}

The wrinkling of edges described above has an interesting connection with the model proposed by Stolarski et al. [51], which is particularly useful to additionally understand that wrinkling alone might be appropriate to avoid rigidification, but it does not suffice to completely describe the deformation, as the zero-energy membrane mode must yield a non-zero bending mode.

The basic idea of this section is to split the deformation into in-plane and out-of-plane displacements, denoted with $u$ and $w$, so that the membrane strain can be written in terms of Föppl-von Karman kinematics [4]

$$
\epsilon=\nabla u+(\nabla w)^{2},
$$


combined with a co-rotational formulation to maintain the frame invariance of the model. The key point to avoid locking is to notice again that the linearized CST energy can be build from the three edge elongations $\eta_{i}:=\sqrt{I_{i} / \bar{I}_{i}}-1$ as

$$
E(\eta)=\sum_{f \in F} \eta_{f}^{T} K_{f} \eta_{f}
$$

where $K_{f}$ is the stiffness matrix and $\eta_{f}$ is the vector containing the three edge elongations relative to the face $f$. Thanks to this observation, it is possible to define

$$
a_{i}:=-\int_{0}^{L_{i}}(\nabla w)^{2} d s \quad \forall i \in E .
$$

so that we obtained the modified energy

$$
\tilde{E}(\eta, a)=\sum_{f \in F}\left(\eta_{f}-a_{f}\right)^{T} K_{f}\left(\eta_{f}-a_{f}\right),
$$

where $a_{f}$ is a vector containing the values relative to the three edges of the face $f$. Specifically, Stolarski et al. use Marguerre's linearization $(\nabla w)^{2} \approx \nabla w_{0} \nabla w$ for efficiency purposes but, more importantly, approximate $w$ using DKT elements, assuming $w=0$ at vertices.

From the kinematic perspective, the resulting model is equivalent to the wrinkling of edges described above and, as such, it does not pass the PPM test, despite performing very well for the popular shell benchmarks [51]. Energetically, however, definition (6.8) introduces a crucial coupling between wrinkling and bending, which has been so far missing in our treatment.

\subsubsection{Geometry of wrinkling}

Let us observe now that the non-rigid solution of SSP produced by the edge wrinkling is, when unwrapped on the plane, exactly identical to its PPM failure. Motivated by this observation, we consider now the projection of the wrinkled triangle onto a different plane, namely, a hinge-based tangent plane.

Let our degrees of freedom be

$$
v_{i=1 . . V} \in \mathbb{R}^{3},
$$

where $\mathrm{V}$ is the total number of vertices in the mesh. We start by looking at the hinge $\left\{v_{1}, v_{2}, v_{3}, v_{4}\right\}$ where $\left\{v_{1}, v_{2}\right\}$ and $\left\{v_{3}, v_{4}\right\}$ denote hinge and flap vertices, respectively. Since it is a known result that the midpoints of the sides of a quadrilateral are the vertices of a parallelogram, we express its normal as

$$
n=\frac{\left(v_{2}-v_{1}\right) \times\left(v_{4}-v_{3}\right)}{\left\|\left(v_{2}-v_{1}\right) \times\left(v_{4}-v_{3}\right)\right\|} .
$$

If the hinge is flat, as in PPM, the resulting model is equivalent to the triangle-based wrinkling and therefore there is no failure mode. On the contrary, for SSP, it will be equivalent to edge-based wrinkling and therefore it does not rigidify. 


\section{Mimetic surfaces}

\section{Wrinkling of hinges}

In this case, we define wrinkling to penalize only the stretching of the projected triangle onto the plane defined by $n$. First, the projected edge lengths can easily be computed as

$$
\epsilon\left(e_{i}\right)=\left\|v_{j}-v_{k}\right\|^{2}-\left|\left(v_{j}-v_{k}\right) \cdot n\right|^{2}, \quad i=(j, k) \in E,
$$

from which the CST strain $\epsilon$ can be computed, whose eigenvalues are $\lambda_{1}$ and $\lambda_{2}$.

Unfortunately, while being exact for two triangles, this model still does not induce the proper bending contribution under compression resulting in, e.g., no failure mode but zero bending energy in the case of PPM. More importantly, the model does not allow the addition of a third triangle coplanar to its neighbor when a hinge is bent. To see this, notice that for a bent hinge the height of a triangle is allowed to increase, but since the projection on a coplanar triangle is the identity, this mode has nonzero membrane contribution. So hinge wrinkling is non-local, i.e., the wrinkling of one hinge restricts the configurational space of its neighbors.

\subsubsection{Tensegrity}

We have so far come to the conclusion that using three constraints per triangle produces either locking or zero-energy modes. The correct way to look at wrinkling is in fact to consider only compressions of extrinsic distances, since discrete intrinsic distances can either increase or decrease, but unfortunately this does not give informations about the deformation regime. In order to capture the compression-induced bending, we increase our set of degrees of freedom to be

$$
v_{i=1 . . V} \in \mathbb{R}^{3}, \quad e_{i=1 . . E} \in \mathbb{R},
$$

where $\mathrm{V}$ and $\mathrm{E}$ are the total number of vertices and edges in the mesh, respectively. The role of the scalar variable $e_{i}$ is to recover the midpoint position

$$
m_{i}=\frac{1}{2}\left(v_{j}+v_{k}\right)+e_{i} n_{i}, \quad i=(j, k) \in E,
$$

where $n$ is a known edge normal. From the knowledge of $m_{i}$, it is straightforward to choose any bending model and apply it on each subtriangle of the original mesh. However, the computation of the membrane contribution of $e_{i}$ raises the question of how to properly define the membrane energy such that there are no overlapping contribution in the case of purely tensional deformations.

$$
I_{3 i+j}:=\left\|m_{j}-v_{i}\right\|^{2}, \quad i, j=0,1,2, \quad i \neq j .
$$

The first naïve idea is to consider the CST model on the subdivided triangle, with the edge wrinkling modification (6.5) only for the inner edges, i.e., those connecting midpoint to midpoint. While very appealing, this idea causes a failure of PPM. 


\section{Wrinkling of dual edges}

Let us consider a hinge $\left\{v_{1}, v_{2}, v_{3}, v_{4}\right\}$ with a defined normal $n$, e.g., the parallelogram normal (6.11). The projected areas of the triangles can be computed as

$$
\begin{aligned}
& A_{1}=\left(\left(v_{1}-v_{3}\right) \times\left(v_{2}-v_{3}\right)\right) \cdot n, \\
& A_{2}=\left(\left(v_{2}-v_{4}\right) \times\left(v_{1}-v_{4}\right)\right) \cdot n,
\end{aligned}
$$

from which we recover the dual edge

$$
\left\|* v_{1} v_{2}\right\|=\frac{A_{1}+A_{2}}{\left\|v_{2}-v_{1}\right\|} .
$$

The first fundamental form can be recovered from its actions along the dual edges

$$
I_{3 i}-\bar{I}_{3 i}=\left\|* v_{j} v_{k}\right\|^{2}-* \bar{L}_{i}^{2}+a_{i}^{2}, \quad i=0,1,2,
$$

where $* \bar{L}$ is the undeformed length of the dual. From this, the membrane energy is computed. This final adjustment repairs the non-locality issues of the hinge wrinkling described above, but it still yields zero bending energy for the compression of PPM.

\section{Wrinkling of medians}

An alternative and simpler view, is to use the tensegrity shown in Fig. 6.4. In this case, projections are not needed, since everything can be computed in terms of extrinsic distances. Thanks to this property, this approach does not produce the PPM failure mode.

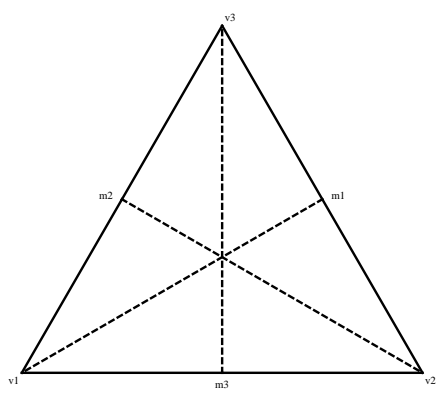

Figure 6.4.: Tensegrity representation of median wrinkling. Dashed lines denote cables, solid lines denote bars.

In this case, the three additional constraints are defined as

$$
I_{3 i}-\bar{I}_{3 i}:=\left\|m_{i}-v_{i}\right\|^{2}-\left\|\bar{m}_{i}-\bar{v}_{i}\right\|^{2}+a_{i}^{2}, \quad i=0,1,2 .
$$

where $a_{i}$ is the wrinkling parameter associated to the vertex $v_{i}$. The three constraints (6.20), together with the six equations (6.15), complete a locking-free kinematic model free of failure modes. 


\subsection{Energy}

We now need to build a membrane energy, whose limit $t \rightarrow 0$ yields the median wrinkling constraints defined above. In order to do this, we propose a novel extension of the piecewise constant fundamental forms presented in Chapter 4 to piecewise discontinuous linear fundamental forms.

\subsubsection{Linear membrane triangle (LMT)}

We have seen in the previous post how to measure inner edges from heights, in order to use a piecewise Constant Strain Triangle (CST) to measure the membrane energy of the element. In order to achieve this, let us consider the DOFs $\left\{v_{i} \in \mathbb{R}^{3}\right\}_{i=1 . . V}$ and $\left\{m_{i} \in \mathbb{R}^{3}\right\}_{i=1 . . E}$, where it is not of our concern now whether the midpoints $m_{i}$ are computed using scalar variables along each edge or they are independent from the vertex positions.
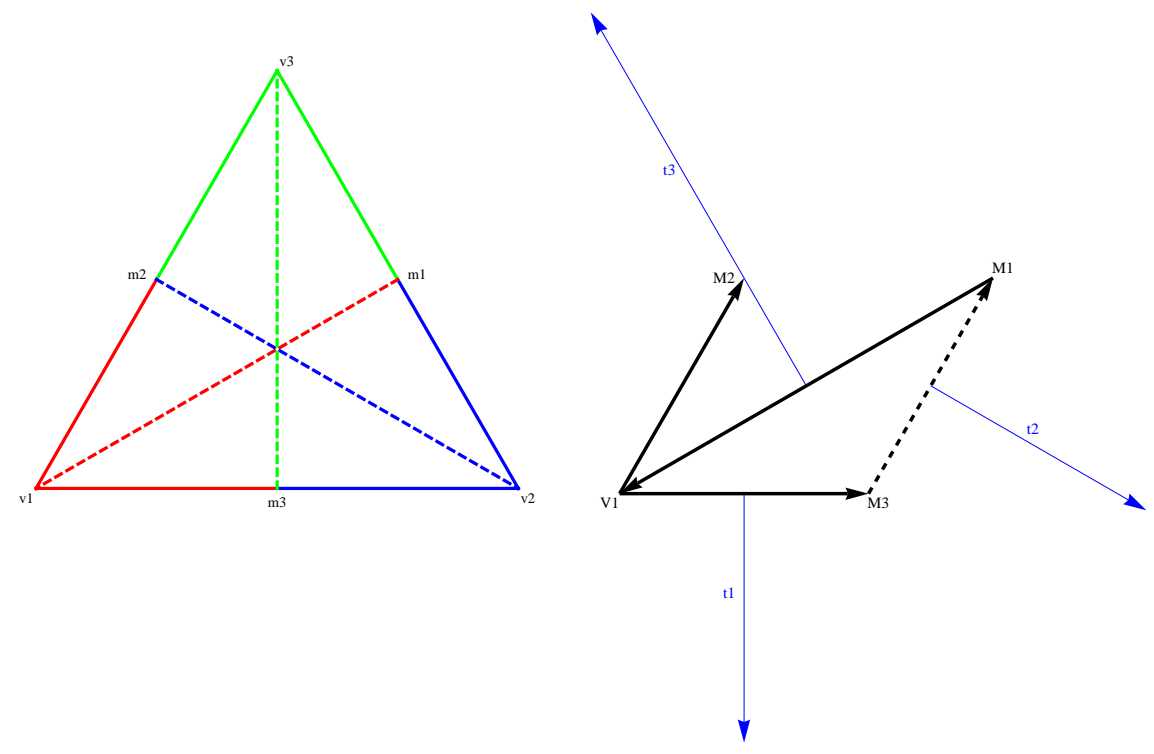

Figure 6.5.: On the left, deformation modes associated to each vertex. On the right, basis vectors (in blue) for the undeformed frame (in black).

We recall the definition of the strain tensor $\epsilon$ as the difference of deformed and undeformed first fundamental forms

$$
\epsilon=\mathrm{I}-\overline{\mathrm{I}} \text {. }
$$

For the sake of simplicity, we concentrate on an energy of the form

$$
E=\int \sigma: \epsilon
$$


where $\epsilon$ and $\sigma$ are the strain and stress tensors. In particular, we assume the following interpolation

$$
\epsilon, \sigma \in \mathbb{P}^{1} \cap L^{2},
$$

which implies that

$$
\epsilon(s, t)=(1-s-t) \epsilon^{1}+s \epsilon^{2}+t \epsilon^{3}
$$

where

$$
\epsilon^{i}=\left(\epsilon_{11}^{i}, \epsilon_{12}^{i}, \epsilon_{21}^{i}, \epsilon_{22}^{i}\right)
$$

is the strain evaluated at the vertex $v_{i}$, which is computed from each frame shown in Fig. 6.5. For each vertex, we use the three vectors denoted with the same color in the figure. Explicitly, this means that each strain can be computed using CST assembled from the action of the first fundamental form on three arbitrary directions as

$$
\epsilon^{i}=\epsilon^{i}\left(I_{i}, I_{i+1}, I_{i+2}\right), \quad i=0,1,2,
$$

where $I_{i}$ are computed from (6.15) and (6.20). By grouping the variables as

$$
\epsilon_{i j}=\left(\epsilon_{i j}^{1}, \epsilon_{i j}^{2}, \epsilon_{i j}^{3}\right),
$$

the discrete energy has the form

$$
E_{h}=\sigma_{11} M \epsilon_{11}+2 \sigma_{12} M \epsilon_{12}+\sigma_{22} M \epsilon_{22}
$$

where we have used the symmetry of the tensors and $M$ is the $\mathbb{P}^{1}$ mass matrix

$$
M=\frac{A}{12}\left(\begin{array}{lll}
2 & 1 & 1 \\
1 & 2 & 1 \\
1 & 1 & 2
\end{array}\right)
$$

Each symmetric $2 \times 2$ tensor has 9 free parameters over a triangle. The 9 parameters of the stress tensor are trivially determined by Hooke's law

$$
\left(\begin{array}{c}
\sigma_{11} \\
\sigma_{22} \\
2 \sigma_{12}
\end{array}\right)=\frac{E}{1-\nu^{2}}\left(\begin{array}{ccc}
1 & \nu & 0 \\
\nu & 1 & 0 \\
0 & 0 & 1-\nu
\end{array}\right)\left(\begin{array}{c}
\epsilon_{11} \\
\epsilon_{22} \\
2 \epsilon_{12}
\end{array}\right)
$$

which implies that

$$
\begin{aligned}
E_{h} & =\frac{E}{1-\nu^{2}}\left(\left(\epsilon_{11}+\nu \epsilon_{22}\right) M \epsilon_{11}+2(1-\nu) \epsilon_{12} M \epsilon_{12}+\left(\epsilon_{22}+\nu \epsilon_{11}\right) M \epsilon_{22}\right) \\
& =\frac{E}{1-\nu^{2}}\left(\epsilon_{11} M \epsilon_{11}+\epsilon_{22} M \epsilon_{22}+2 \nu \epsilon_{11} M \epsilon_{22}+2(1-\nu) \epsilon_{12} M \epsilon_{12}\right) .
\end{aligned}
$$




\section{Mimetic surfaces}

\section{Tying points}

The above framework can be generalized even further, using an idea stemming from the MITC element, presented in Chapter 4. As we will see in the next section, our LMT produces a slightly softer solution in $2 \mathrm{D}$ membrane problem. To correct this problem, rather than assuming that the piecewise constant strains correspond to the piecewise linear strain when evaluated at the vertices of the coarse triangle, we enforce the condition

$$
\epsilon\left(s_{i}, t_{i}\right):=\epsilon^{i}, \quad i=1,2,3,
$$

where $\left(s_{i}, t_{i}\right)$ are carefully chosen tying points inside the coarse triangle. In the case $\left(s_{1}, t_{1}\right)=(0,0),\left(s_{2}, t_{2}\right)=(1,0),\left(s_{3}, t_{3}\right)=(0,1)$, then the coupling is equivalent to the particular case of a standard FE projection. From a discrete viewpoint, a better choice is

$$
\left(s_{1}, t_{1}\right)=\left(\frac{1}{4}, \frac{1}{4}\right),\left(s_{2}, t_{2}\right)=\left(\frac{1}{2}, \frac{1}{4}\right),\left(s_{3}, t_{3}\right)=\left(\frac{1}{4}, \frac{1}{2}\right),
$$

which corresponds to choosing the centroids of the parallelograms defined by each frame in Fig. 6.5. In practice, we have found that choices closer to the centroid of the coarse triangle can give even better results.

\section{Discrete linear strain triangle (dLST)}

We here remark that this energetic framework is not constrained to be applied only with the kinematics defined on the previous section. To outline this, we now present an alternative formulation, which turns out to be equivalent to the choice

$$
\mathbf{x} \in \mathbb{P}^{1} i s o \mathbb{P}^{2}, \quad \epsilon \in \mathbb{P}_{0}^{1}:=\mathbb{P}^{1} \cap L^{2},
$$

in the Hellinger-Reissner mixed formulation presented in Chapter 3. Moreover, this formulation generalizes the linear strain triangle (LST), which is obtained with the choice

$$
\mathbf{x} \in \mathbb{P}^{2}, \quad \epsilon \in \mathbb{P}_{0}^{1},
$$

in the Hellinger-Reissner mixed formulation, which is in turn equivalent to the choice $\mathbf{x} \in \mathbb{P}^{2}$ in the primal formulation of $2 \mathrm{D}$ elasticity, after having observed than $\epsilon$ can be eliminated from the unknowns.

Our formulation is obtained by simply replacing equation (6.32) with

$$
\int_{\overline{\mathcal{S}}} \epsilon_{i j} \phi d s d t=\sum_{k=1}^{3} \int_{\overline{\mathcal{S}}} \epsilon_{i j}^{k} \phi d s d t, \quad \forall \phi \in \mathbb{P}^{1}, \quad i, j=1,2,
$$

where we assume $\epsilon_{i j}^{k}$ to be zero outside the parallelogram defined by its corresponding frame. Clearly, this is nothing but a FE projection between the spaces indicated in (6.34). While suffering from locking, we will show in the next section that this new formulation has an exceptional performance in the context of $2 \mathrm{D}$ membranes. To our knowledge, this choice has never been applied and represent a new result of this work. It remains an open problem, however, whether is possible to define an appropriate wrinkling kinematics in the case of shells. 


\subsubsection{Linear curvature triangle (LCT)}

We have introduced in Chapter 4 the Midedge Shape Operator (MSO) [25], arising from a piecewise constant approximation of the second fundamental form. As we have seen, MSO adds a rotation angle per edge to the CST description of the deformation. Motivated by already having the midpoint position available in our membrane model, we here propose a piecewise linear approximation of the curvature which is free from rotations.

As discussed in Chapter 4 for the case of Constant Shells (CS), the idea of eliminating the rotation from the DOFs is not new. This can be done, e.g, by defining the normal vector field $n$ at each edge to be the average of the two adjacent face normals. This effectively halves the total number of DOFs and performs well if the mesh is regular, but leads to more mesh dependence and stiffer results in case of free edges [21]. However, we will see that our formulation outperforms MSO on a regular mesh with free edges.

Following step by step the derivation of LMT, let us consider the DOFs $\left\{v_{i} \in \mathbb{R}^{3}\right\}_{i=1 . . V}$ and $\left\{m_{i} \in \mathbb{R}^{3}\right\}_{i=1 . . E}$. We define the bending strain tensor $\kappa$ as the difference of deformed and undeformed second fundamental forms

$$
\kappa:=\mathrm{II}-\overline{\mathrm{II}} \text {. }
$$

For the sake of simplicity, we concentrate on an energy of the form

$$
E=\int_{\overline{\mathcal{S}}} \sigma: \kappa,
$$

where $\kappa$ and $\sigma$ are the strain and stress tensors. In particular, we assume the following interpolation

$$
\kappa, \sigma \in \mathbb{P}^{1} \cap L^{2},
$$

which implies that

$$
\kappa(s, t)=(1-s-t) \kappa^{1}+s \kappa^{2}+t \kappa^{3}
$$

where

$$
\kappa^{i}=\left(\kappa_{11}^{i}, \kappa_{12}^{i}, \kappa_{21}^{i}, \kappa_{22}^{i}\right)
$$

is the strain evaluated at the vertex $v_{i}$. As done in equation (6.36), we do not use the frames indicated in Fig. 6.5, but rather project the strains obtained with the following FE choice

$$
\mathbf{n} \in \mathbb{C} \mathbb{R} i \text { so } \mathbb{P}^{2}, \quad \kappa \in \mathbb{P}_{0}^{1}:=\mathbb{P}^{1} \cap L^{2},
$$

where we use $\mathbb{C R}$ iso $\mathbb{P}^{2}$ to denote $\mathbb{C R}$ elements applied on the refined mesh. This choice is equivalent to interpolating linearly on the coarse triangle the four piecewise constant second fundamental forms, which are computed on each subtriangle.

By grouping the variables as

$$
\kappa_{i j}=\left(\kappa_{i j}^{1}, \kappa_{i j}^{2}, \kappa_{i j}^{3}\right),
$$

the discrete energy has the form

$$
E_{h}=\sigma_{11} M \kappa_{11}+2 \sigma_{12} M \kappa_{12}+\sigma_{22} M \kappa_{22},
$$




\section{Mimetic surfaces}

where we have used the symmetry of the tensors and $M$ is the $\mathbb{P}^{1}$ mass matrix

$$
M=\frac{A}{12}\left(\begin{array}{lll}
2 & 1 & 1 \\
1 & 2 & 1 \\
1 & 1 & 2
\end{array}\right) .
$$

Each symmetric $2 \times 2$ tensor has 9 free parameters over a triangle. The 9 parameters of the stress tensor are trivially determined by Hooke's law

$$
\left(\begin{array}{c}
\sigma_{11} \\
\sigma_{22} \\
2 \sigma_{12}
\end{array}\right)=\frac{E}{1-\nu^{2}}\left(\begin{array}{ccc}
1 & \nu & 0 \\
\nu & 1 & 0 \\
0 & 0 & 1-\nu
\end{array}\right)\left(\begin{array}{c}
\kappa_{11} \\
\kappa_{22} \\
2 \kappa_{12}
\end{array}\right),
$$

which implies that

$$
\begin{aligned}
E_{h} & =\frac{E}{1-\nu^{2}}\left(\left(\kappa_{11}+\nu \kappa_{22}\right) M \kappa_{11}+2(1-\nu) \kappa_{12} M \kappa_{12}+\left(\kappa_{22}+\nu \kappa_{11}\right) M \kappa_{22}\right) \\
& =\frac{E}{1-\nu^{2}}\left(\kappa_{11} M \kappa_{11}+\kappa_{22} M \kappa_{22}+2 \nu \kappa_{11} M \kappa_{22}+2(1-\nu) \kappa_{12} M \kappa_{12}\right) .
\end{aligned}
$$

\section{Boundary conditions}

As anticipated, the case of free edges can be a source of problems, if BCs are applied naïvely. We here discuss the case of MSO, since our treatment can be extended to each subtriangle of LBT. For the corresponding rotation-free FE, this was discussed in detail in [42], from which we recall Fig. 6.6. In the figure, the constant curvature triangle is $\left(v_{4}, v_{5}, v_{6}\right)$, while the other three triangles are used to compute the face normals.

By inserting a fictitious node, we are in fact introducing rotations only along boundary edges. This is, in turn, equivalent to prescribing $\operatorname{II}\left(v_{4}-v_{6}\right)=0$. Physically, this condition makes the curvature tensor rank-deficient, which implies that there is no bending moment around the free edge.

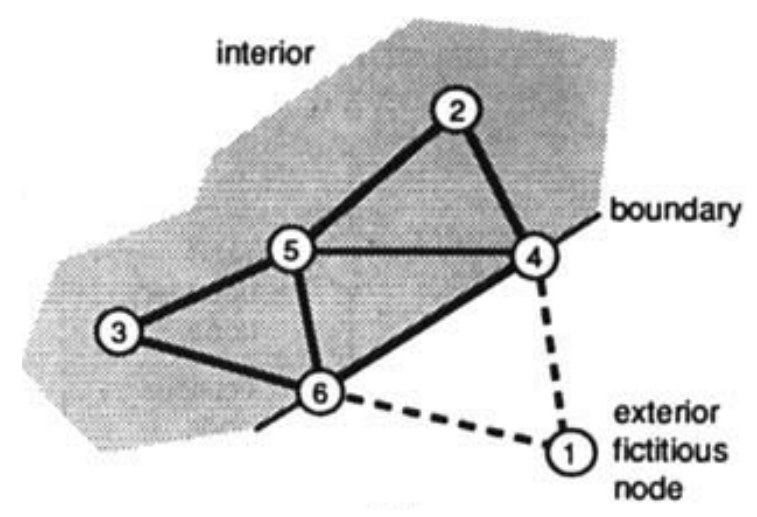

Figure 6.6.: Illustration of free edge boundary conditions, as depicted by [42]. 
Our modification consists in enforcing a similar condition in $\left(v_{3}, v_{6}, v_{5}\right)$ and $\left(v_{2}, v_{5}, v_{4}\right)$, which normally would be excluded from boundary computations. In these triangles, we define

$$
\mathrm{II}\left(v_{3}-v_{5}\right)=\operatorname{II}\left(v_{5}-v_{2}\right)=0,
$$

which makes the bending moment consistent with the neighboring boundary triangles. As we will see in the following section, this choice has a dramatic impact on the accuracy of free-edge computations, making this element clearly more competitive than MSO on regular meshes. Finally, in the case of clamped BCs, it suffices to prescribe the edge normal $n$ at clamped boundaries.

\subsection{Numerical experiments}

We test our new discrete shell theory on the following aspects:

- The approximation of membrane-dominated deformations, for which we use a $2 \mathrm{D}$ test taken from [20] and shown in Fig. 6.7.

- The approximation of bending-dominated deformations, for which we use standard plate bending benchmark problems [52], shown in Fig. 6.9.

- The absence of locking, for which we use irregular meshes with the simple supported plate and the visual quality of draping using very coarse meshes.

We will obtain clear evidence that the discrete shell theory proposed in this chapter is free of locking and suitable for the approximation of any kind of deformation regime that shells can exhibit.

\section{Cantilever under shear load}

We consider the shear-loaded cantilever illustrated in Fig. 6.7, clamped on the left side, where the squares indicate areas which will be subdivided into two triangles. The parabolic load is obtained by lumping the corresponding integral on each vertex.

We use this example in order to compare the accuracy of the following discrete membrane theories:

- CST: the Constant Strain Triangle.

- LST: the Linear Strain Triangle, which in the context of small-deformations elasticity means the choice $\phi \in \mathbb{P}^{2}$ in the elastic energy (3.45) formulated in terms of displacements.

- ALL: the Allman triangle with a 3-point integration rule, based on the computation of a linear strain field from the vertices of the triangle and the midpoint of the DKT surface. 
a)

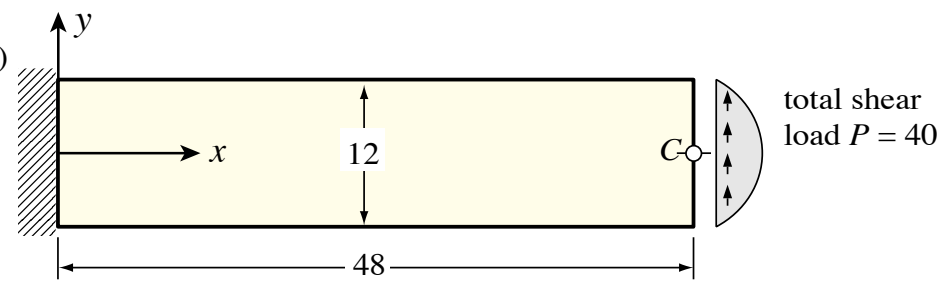

b)

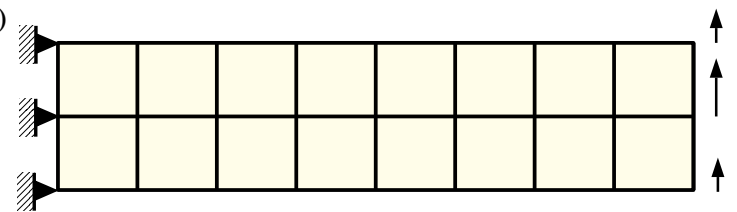

Figure 6.7.: Illustration of the cantilever under shear load [20], for a 8x2 mesh.

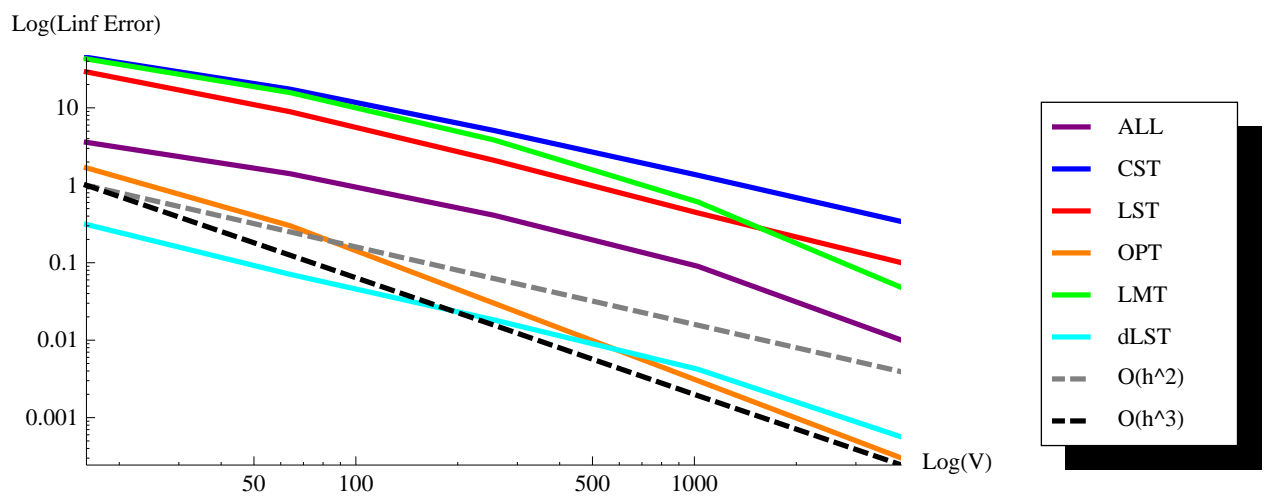

Figure 6.8.: Shear-loaded cantilever: logarithmic plot of the error in the in-plane deflection at the point $\mathrm{C}$ (see Fig. 6.7) versus the total number of vertices $V$ in the mesh. Values for ALL, CST, OPT, and LST, are taken from [20].

- OPT: the element proposed by Felippa [20], based on the optimal choice of constant in a template model consisting in the weighted sum of a CST and a higher-order deformation modes.

- LMT: our novel discrete shell theory presented in this chapter, with strains evaluated at barycentric coordinates $(0.4,0.3,0.3)$.

- dLST: the discrete linear strain triangle, proposed in equation (6.34).

As we can see from Fig. 6.8, OPT and dLST outperform all the other elements. However, dLST converges to a $0.001 \%$ softer solution, which could be caused by the fact that we have used a geometrically nonlinear strain for our experiments, while the 
numbers provided by [20] are obtained using its linearized version, which penalizes also rigid rotations.

We remark that, for this test, we have not considered wrinkling of medians and we do not constrain midpoints to move along the angle-bisecting normal direction. The reason is that we want to test our novel energetic model, independently from the kinematic assumptions. If we had incorporated them, then LMT would reduce to CST for in-plane membrane deformations.

\section{Cantilever under transverse load}

This example measures the accuracy of the representation of curvature and it is not subjected to membrane locking, if the mesh is obtaining by subdivision of the quadrilaterals shown in Fig. 6.9.

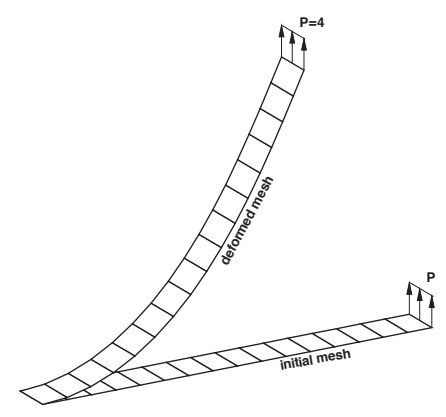

Figure 6.9.: Illustration of the deformation of the loaded cantilever and the slit annular plate, taken from [52].

Since the membrane model is uninfluential, we use the same membrane model to compare the following discrete bending theories:

- MSO: the midedge shape operator, presented in Chapter 4.

- ROT: the rotation-free version of MSO, using angle-bisecting normals

- S4R: the four-noded element with shear strain and reduced integration, which can be found in Abaqus [52].

- LCT: our novel linear curvature triangle, presented in this chapter.

The results can be seen in Fig. 6.10. We plot the relative $L^{\infty}$ error versus the number of DOFs, which is a direct indication of the computational cost. We see that our formulation (LCT) is superior even to the MSO model. Interestingly, the MSO model slightly overestimates the solution at coarse scales, while our method underestimates it, which is a clear indication that MSO could not benefit from our new BCs. 

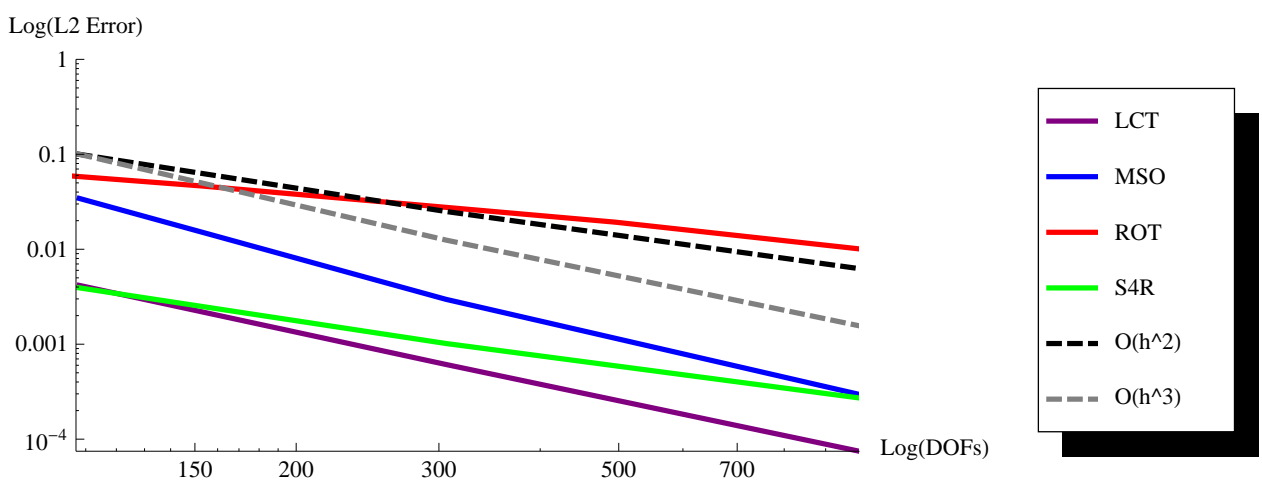

Figure 6.10.: Loaded cantilever: logarithmic plot of the error in the out-of-plane deflection at the loaded tip (see Fig. 6.9) versus the total number of DOFs used by each method. The reference solution is given in [52].

\section{Supported plate}

In this example, we test the supported plate shown in Fig. 6.2, using the meshes shown in Fig. 6.11. In the case of a regular mesh, we perform two experiments: first the situation in which the edges are aligned with the rulings of the smooth solution, refer to as the aligned case, then the situation in which they are orthogonal, refer to as the opposite case in Fig. 6.12.
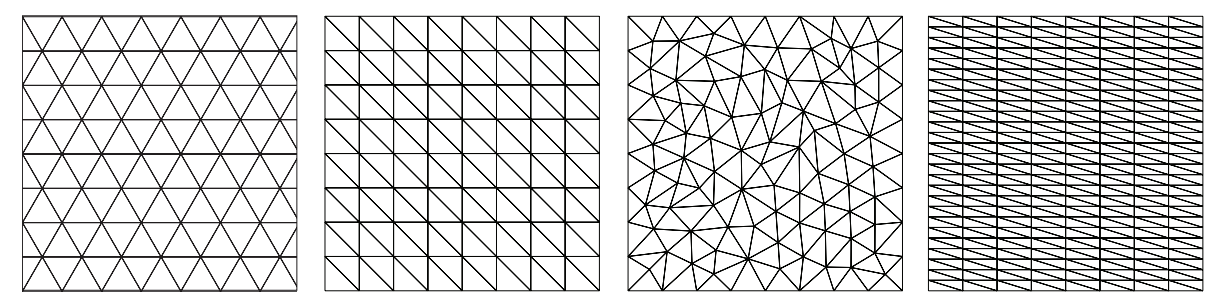

Figure 6.11.: Meshes used for testing the simply supported plates. From left to right: equilateral, regular, irregular, and aspect ratio.

In Fig. 6.12, we plot the $L^{\infty}$ norm of the solution versus the number of DOFs for the tested meshes, which clearly shows no locking. As a comparison, we have included the behavior of CST and dLST when a regular mesh having edges orthogonal to the smooth rulings is employed. We remark that, to our knowledge, such remarkable result cannot be found anywhere in the literature of membrane locking. 

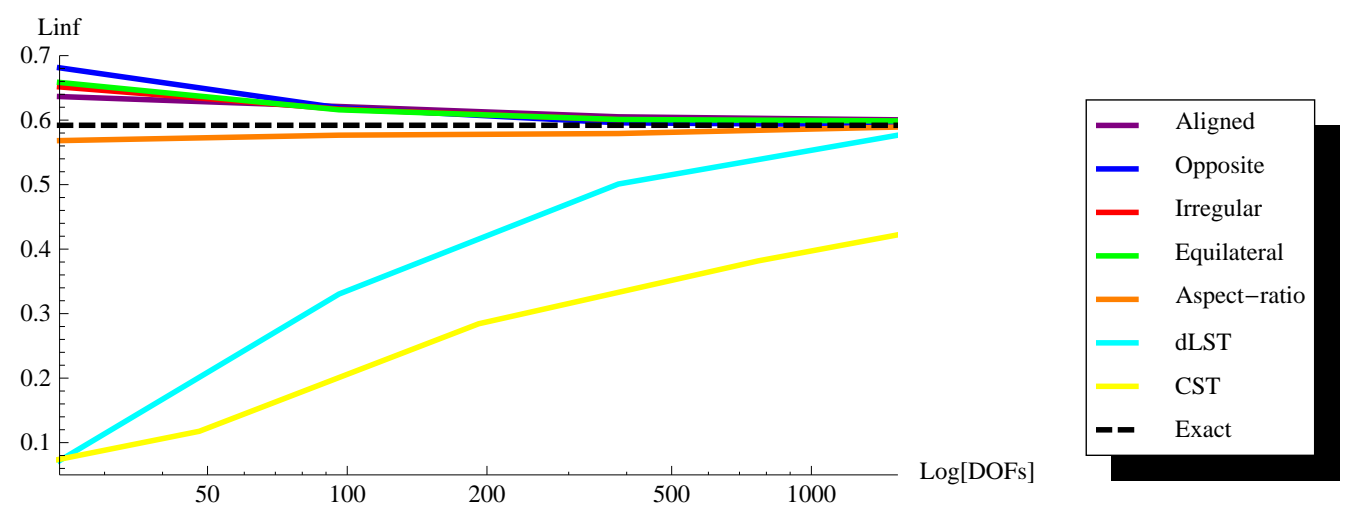

Figure 6.12.: $L^{\infty}$ norm of the vertical displacement versus number of DOFs. dLST and CST were tested on the opposite mesh, i.e., a regular mesh in which the edges are orthogonal to the rulings of the smooth solution. We used a membrane-to-bending stiffness ratio of $10^{7}$.

\section{Draping over a cube}

As a last example, we compare qualitatively the visual accuracy in simulating draping over a cube using an extremely coarse mesh of $4 \times 4$ vertices, which is a typical scenario for a rapid preview of a cloth simulation in computer graphics.
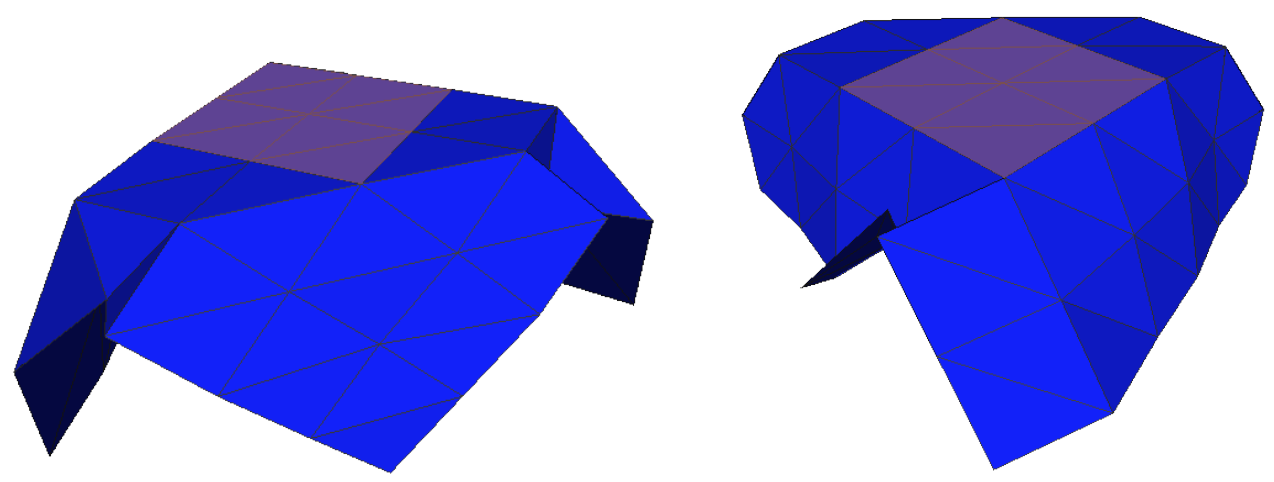

Figure 6.13.: Draping of a cloth over a square object, highlighted in the figure. Two different perspectives of the result obtained with CST.

We compare the following discrete models:

- CST: in this case, we used a refined $8 \times 8$ version of the mesh, in order to have a fair comparison in terms of total number of nodes. As can be seen in Fig. 6.13, locking is severe and the final solution exhibits a very strong mesh dependence. 

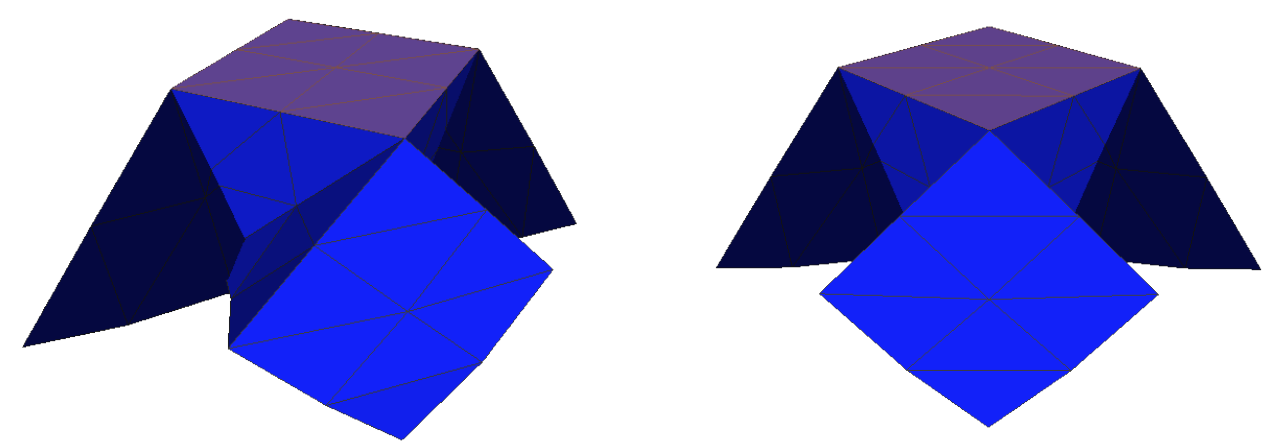

Figure 6.14.: Draping of a cloth over a square object, highlighted in the figure. Two different perspectives of the result obtained with $\mathbb{P}^{1}$ iso $\mathbb{P}^{2}-\mathbb{P}_{0}^{1}$ elements.
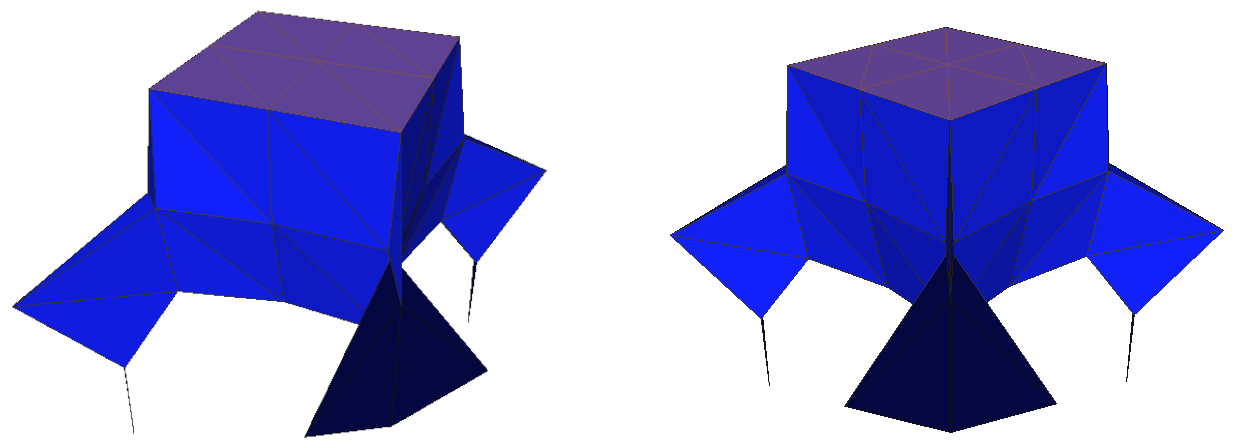

Figure 6.15.: Draping of a cloth over a square object, highlighted in the figure. Two different perspectives of the result obtained with the model by Stolarski et al. [51].

- dLST: in this case, we used the $\mathbb{P}^{1}$ iso $\mathbb{P}^{2}-\mathbb{P}_{0}^{1}$ elements, which have shown an excellent behavior in a membrane-dominated case. As can be seen in Fig. 6.14, these elements lock in a bending-dominated scenario. Indeed, notice that coarse triangles, formed by groups of four triangles shown in the picture, remain nearly flat.

- STO: in this case, we have completely removed the medians and preserved only the length of the half-edges of the triangles. The resulting kinematic description is very similar to the model by Stolarski et al. [51], which we have presented earlier. As can be seen in Fig. 6.15, locking is not present but it is replaced by the existence of zero-energy membrane modes which cause undesirable artifacts in the corner nodes of the mesh, producing an overall unrealistic result. 

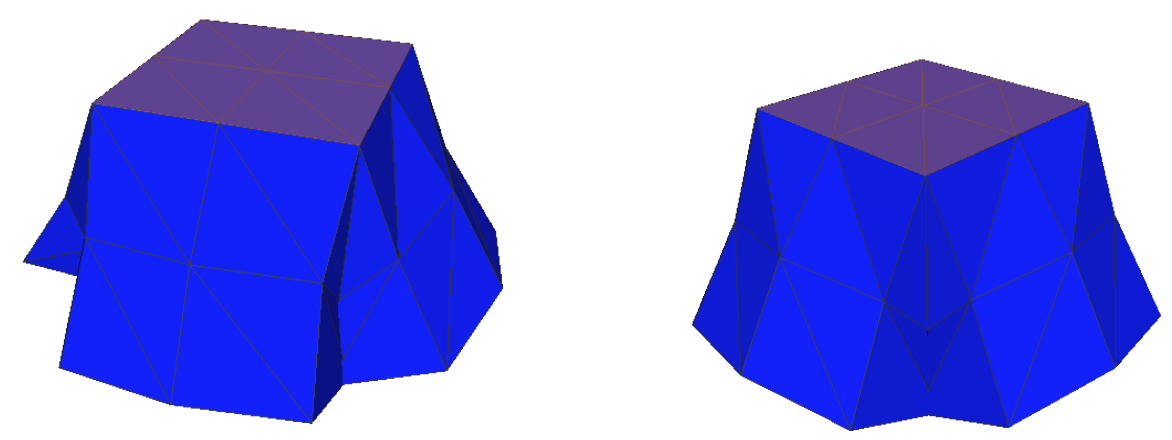

Figure 6.16.: Draping of a cloth over a square object, highlighted in the figure. Two different perspectives of the result obtained with median wrinkling.

- LMT: as can be seen in Fig. 6.16, our model is free of locking and unrealistic artifacts, producing a very symmetric and realistic solution.

We remark that in all the pictures we have rendered the simplex connecting of all the nodes of the original $4 \times 4$ triangulation, which results in a $8 \times 8$ mesh. It is clear from this example that our mimetic shell theory produces a dramatic improvement over other methods, providing reliable qualitatively result even on extremely coarse meshes and complicated large deformations scenarios.

\section{Numerical issues}

To conclude, we point out that using directly (6.20) can cause severe problems to Newtonlike solvers, since the initial configuration lies often very close to several bifurcation points. To see this, notice that in order to start wrinkling we need an initial increase in the bending energy, which is often discarded by the solver, resulting in a rigidified local minimum. To avoid this problem, we have devised the following algorithm:

1. Compute a trial solution with $\epsilon_{i i}=0$ in (6.20).

2. Loop over each triangle, if $\epsilon_{i i}>0$, then set $a_{i}=0$ in (6.20).

3. Compute the final wrinkled solution.

The advantage of the above algorithm is manifold: it reduces the total number of iterations, it avoids local minima, and it significantly reduces the number of DOFs, since there is no need to retain $a_{i}$ as a part of the unknowns. 



\section{Conclusions}

Starting from the issue of membrane locking, arising in the FE approximation of shells, we took a step back and looked at the construction of smooth shell theories, describing the deformation of elastic surfaces. In particular, we concentrated on two known but largely overlooked aspects in the transition from smooth to discrete theories:

- The relationship between the kinematic assumptions and the choice of FE spaces.

- The preservation of the correct asymptotic regimes.

The path to detect the importance of the two topics above has been nonlinear. In fact, the original goal of this work was to study the approximation of developable surfaces proposed in [17], for which we found the presence of failure modes and locking. To tackle these issues, we created the strategy of edgepoints. Later, we recognized that this is only a subset of a larger class of surfaces with adaptive kinematics which, however, proved to introduce more issues than benefits.

Only when we started considering the combinatorial adaptation of the mesh, we realized the importance of the preservation of the correct asymptotic regimes in the discrete setting. Motivated by this observation, we decided to formulate a discrete shell theory from first principles, having the property to recover membrane theories and developable surfaces, according to the physics of the problem.

Three main tools were fundamental to achieve this:

- The differential geometry description of strains as the fundamental forms of the surface, computed by defining their action on three linearly independent vectors, which permitted us to avoid the use of a polynomial interpolation of the surface.

- The use of the wrinkling concept [34], which in the past was restricted to membranes, to neglect the artificial membrane contribution during compression.

- The linear interpolation of strains and curvatures over each triangle, which opened up the possibility for more complicated discrete kinematic assumptions.

Therefore, the contribution of this work has been to combine material from graphics, engineering, and mathematics, into a common language, proposing an extension of the CST-MSO discrete theory [25], having piecewise constant strains and curvatures, to a locking-free discrete shell theory with piecewise linear fundamental form, which proved to be beneficial for the examples presented in this thesis. 


\section{Conclusions}

\section{Future work}

The proposed methodology is still in its infancy in computational mechanics, despite having proved to exhibit excellent results in the case of rods [10]. Here, we propose some tasks which can stem from this work:

- The generalization to curved undeformed configurations.

- The extension to higher-order membrane and bending strains.

- The analysis of discrete boundary layers.

- The interpretation of discrete shell theories using FE, which could lead to theoretical results in the convergence analysis.

As it has been the case for computational electromagnetics [50] and fluid dynamics [41], we hope that our effort has shown the potential of relying on physical considerations in the development of the approximation of smooth theories, which are historically tackled using purely analytical concepts, such as polynomial interpolation. In this respect, we believe that our approach towards the formulation of discrete theories, based on defining discrete physical laws on a finite-size element, is more closely related to the formulation of smooth physical laws on infinitesimal elements, rather than to their approximation. 


\section{A. Derivatives}

\section{Membrane derivatives}

We here present the derivation of the CST energy with respect of the undeformed vertices, required in order to compute the gradients and the Hessians of r-adaptivity.

\section{Area}

Assume to have an energy of the form:

$$
E=\frac{1}{A^{n}} W
$$

Then, it is straightforward to obtain gradient and Hessian:

$$
\begin{aligned}
\nabla E= & \nabla\left(\frac{1}{A^{n}}\right) W+\frac{1}{A^{n}} \nabla W=-\frac{n W}{A^{n+1}} \nabla A+\frac{1}{A^{n}} \nabla W \\
\nabla^{2} E= & \nabla^{2}\left(\frac{1}{A^{n}}\right) W+\nabla\left(\frac{1}{A^{n}}\right) \nabla W+\nabla W \nabla\left(\frac{1}{A^{n}}\right)+\frac{1}{A^{n}} \nabla^{2} W \\
= & \frac{n(n+1) W}{A^{n+2}} \nabla A^{T} \nabla A-\frac{n W}{A^{n+1}} \nabla^{2} A- \\
& \frac{n}{A^{n+1}}\left(\nabla A^{T} \nabla W+\nabla W^{T} \nabla A\right)+\frac{1}{A^{n}} \nabla^{2} W .
\end{aligned}
$$

Moreover, we can compute:

$$
\begin{aligned}
(\nabla A)_{p} & =\partial_{v_{p}} A=\frac{t_{p}}{2}=-\frac{n \times e_{p}}{4 A}, \\
\left(\nabla^{2} A\right)_{p q} & =\left(-\nabla\left(\frac{n \times e_{p}}{4 A}\right)\right)_{q} \\
& =-\left(\frac{\nabla\left(n \times e_{p}\right)}{4 A}-\frac{\left(n \times e_{p}\right) \nabla A}{4 A^{2}}\right)_{q}=\frac{\left(-\nabla\left(n \times e_{p}\right)\right)_{q}}{4 A}-\frac{\left(\nabla A^{T} \nabla A\right)_{p q}}{A}, \\
\left(\nabla\left(n \times e_{p}\right)\right)_{q} & =e_{p} \cdot e_{q} \delta_{p q}^{3}+\left(e_{p} \otimes e_{q}-2 e_{q} \otimes e_{p}\right), \quad q \geq p,
\end{aligned}
$$

where $\delta_{p q}^{3}$ is a $3 \times 3$ matrix, having the Kronecker delta $\delta_{p q}$ on its diagonal.

\section{Energy}

Let us denote the vertex positions with $v_{i}$ and their opposite edge vectors as $e_{i}$. Define:

$$
\operatorname{Tr}(Q)=-\sum_{i}\left(e_{j} \cdot e_{k}\right) Q_{i}, \quad \operatorname{Det}(Q)=\sum_{i}\left(Q_{j}+Q_{k}-Q_{i}\right) Q_{i}, \quad Q_{i}=l_{i}^{2}-\bar{l}_{i}^{2},
$$




\section{A. Derivatives}

where $(i, j, k)$ is a circular permutation of $(1,2,3)$. For simplifying the notations, we consider $v_{i}$ and $Q_{i}$ as two separate variables. The energy is:

$$
E=\int_{T}\left(\frac{\operatorname{Tr}(Q)^{2}}{16 A^{4}}-\frac{\operatorname{Det}(Q)}{8 A^{2}}\right) d A=\frac{\operatorname{Tr}(Q)^{2}}{16 A^{3}}-\frac{\operatorname{Det}(Q)}{8 A}=\frac{W_{2}}{16 A^{3}}-\frac{W_{1}}{8 A} .
$$

With $n_{1}=1$ and $n_{2}=3$, we immediately get the gradient:

$$
\begin{aligned}
\nabla E_{1} & =-\frac{\nabla A}{8 A^{2}} W_{1}+\frac{1}{8 A} \nabla W_{1}, \\
\nabla E_{2} & =-\frac{3 \nabla A}{16 A^{4}} W_{2}+\frac{1}{16 A^{3}} \nabla W_{2}, \\
\nabla E & =\left(\frac{1}{8 A^{2}} W_{1}-\frac{3}{16 A^{4}} W_{2}\right) \nabla A+\left(\frac{1}{16 A^{3}} \nabla W_{2}-\frac{1}{8 A} \nabla W_{1}\right) .
\end{aligned}
$$

and the Hessian:

$$
\begin{aligned}
8 \nabla^{2} E_{1} & =\frac{2 W_{1}}{A^{3}} \nabla A^{T} \nabla A-\frac{W_{1}}{A^{2}} \nabla^{2} A-\frac{1}{A^{2}}\left(\nabla A^{T} \nabla W_{1}+\nabla W_{1}^{T} \nabla A\right)+\frac{1}{A} \nabla^{2} W_{1}, \\
16 \nabla^{2} E_{2} & =\frac{12 W}{A^{5}} \nabla A^{T} \nabla A-\frac{3 W_{2}}{A^{4}} \nabla^{2} A-\frac{3}{A^{4}}\left(\nabla A^{T} \nabla W_{2}+\nabla W_{2}^{T} \nabla A\right)+\frac{1}{A^{3}} \nabla^{2} W_{2}, \\
\nabla^{2} E & =\nabla A^{T} \nabla A\left(\frac{3}{4 A^{5}} W_{2}-\frac{1}{4 A^{3}} W_{1}\right)+\nabla^{2} A\left(\frac{1}{8 A^{2}} W_{1}-\frac{3}{16 A^{4}} W_{2}\right) \\
& +\frac{1}{8 A^{2}}\left(\nabla A^{T} \nabla W_{1}+\nabla W_{1}^{T} \nabla A\right)-\frac{3}{16 A^{4}}\left(\nabla A^{T} \nabla W_{2}+\nabla W_{2}^{T} \nabla A\right) \\
& +\left(\frac{1}{16 A^{3}} \nabla^{2} W_{2}-\frac{1}{8 A} \nabla^{2} W_{1}\right) .
\end{aligned}
$$

\section{Determinant}

We have that:

$$
\begin{aligned}
\nabla W_{1} & =\frac{\partial}{\partial v} W_{1}=\frac{\partial Q}{\partial v} \frac{\partial W_{1}}{\partial Q} \\
\nabla^{2} W_{1} & =\frac{\partial^{2}}{\partial v^{2}} W_{1}=\frac{\partial}{\partial v}\left(\frac{\partial Q}{\partial v} \frac{\partial W_{1}}{\partial Q}\right)=\frac{\partial^{2} Q}{\partial v^{2}} \frac{\partial W_{1}}{\partial Q}+\frac{\partial Q}{\partial v} \frac{\partial^{2} W_{1}}{\partial Q^{2}} \frac{\partial Q}{\partial v} .
\end{aligned}
$$

Moreover:

$$
\left(\frac{\partial}{\partial Q} W_{1}\right)_{p}=2\left(Q_{j}+Q_{k}-Q_{p}\right)
$$

\section{Trace}

We have that:

$$
\begin{aligned}
\nabla W_{2} & =\frac{\partial}{\partial v} W_{2}=\frac{\partial Q}{\partial v} \frac{\partial}{\partial Q} W_{2}+\frac{\partial}{\partial v} W_{2}, \\
\nabla^{2} W_{2} & =\frac{\partial^{2}}{\partial v^{2}} W_{2}=\left(\frac{\partial^{2} Q}{\partial v^{2}} \frac{\partial W_{2}}{\partial Q}+\frac{\partial Q}{\partial v} \frac{\partial^{2} W_{2}}{\partial Q^{2}} \frac{\partial Q}{\partial v}\right)+\frac{\partial^{2} W_{2}}{\partial v^{2}}+\frac{\partial Q}{\partial v} \frac{\partial^{2} W_{2}}{\partial v \partial Q}+\frac{\partial^{2} W_{2}}{\partial Q \partial v} \frac{\partial Q}{\partial v} .
\end{aligned}
$$


Moreover:

$$
\begin{aligned}
\left(\frac{\partial}{\partial v} \operatorname{Tr}(Q)\right)_{p}= & -\sum_{i} Q_{i}\left(e_{j} \cdot \partial_{v_{p}}\left(v_{j}-v_{i}\right)+e_{k} \cdot \partial_{v_{p}}\left(v_{i}-v_{k}\right)\right) \\
= & -\sum_{i} Q_{i}\left(e_{j}\left(\delta_{j p}-\delta_{i p}\right)+e_{k}\left(\delta_{i p}-\delta_{k p}\right)\right) \\
= & -\sum_{i} Q_{i}\left(\delta_{i p}\left(e_{k}-e_{j}\right)+e_{j} \delta_{j p}-e_{k} \delta_{k p}\right) \\
= & Q_{p}\left(e_{j}-e_{k}\right)+e_{p}\left(Q_{j}-Q_{k}\right), \\
\left(\frac{\partial}{\partial v} W_{2}\right)_{p}= & 2 \operatorname{Tr}(Q)\left(\frac{\partial}{\partial v} \operatorname{Tr}(Q)\right)_{p}=2 \operatorname{Tr}(Q)\left(Q_{p}\left(e_{j}-e_{k}\right)+e_{p}\left(Q_{j}-Q_{k}\right)\right), \\
\left(\frac{\partial}{\partial Q} W_{2}\right)_{p}= & 2 \operatorname{Tr}(Q)\left(-\sum_{i}\left(e_{j} \cdot e_{k}\right) \nabla Q_{i}\right)_{p}=-2 \operatorname{Tr}(Q)\left(e_{j} \cdot e_{k}\right), \\
\left(\frac{\partial^{2}}{\partial Q \partial v} W_{2}\right)_{p q}= & 2 \operatorname{Tr}(Q)\left(\delta_{p q}\left(e_{j}-e_{k}\right)+e_{p}\left(\delta_{q j}-\delta_{q k}\right)\right)- \\
& 2\left(e_{r} \cdot e_{s}\right)\left(Q_{p}\left(e_{j}-e_{k}\right)+e_{p}\left(Q_{j}-Q_{k}\right)\right), \\
\left(\frac{\partial^{2}}{\partial v^{2}} W_{2}\right)_{p q}= & 2\left(\frac{\partial}{\partial v} \operatorname{Tr}(Q)\right)_{p}\left(\frac{\partial}{\partial v} \operatorname{Tr}(Q)\right)_{q}+ \\
& 2 \operatorname{Tr}(Q)\left(Q_{p} \partial_{v_{q}}\left(e_{j}-e_{k}\right)+\left(Q_{j}-Q_{k}\right) \partial_{v_{q}} e_{p}\right) \\
= & 2\left(\frac{\partial}{\partial v} \operatorname{Tr}(Q)\right)_{p}\left(\frac{\partial}{\partial v} \operatorname{Tr}(Q)\right)_{q}+ \\
& 2 \operatorname{Tr}(Q)\left(\delta_{p q} Q_{p}-\delta_{k q}\left(Q_{p}-Q_{j}+Q_{k}\right)-\delta_{j q}\left(Q_{p}+Q_{j}-Q_{k}\right)\right),
\end{aligned}
$$

where $(p, j, k)$ and $(q, r, s)$ are cyclic permutations of $(1,2,3)$.

\section{Force}

The total force $F$ is obtained by augmenting the standard CST force $F_{C S T}$ :

$$
\begin{aligned}
F & =-\nabla E=-\frac{\partial Q}{\partial v} \frac{\partial E}{\partial Q}-\frac{\partial E}{\partial v}=F_{C S T}-\frac{\partial E}{\partial v}, \\
\frac{\partial E}{\partial Q} & =\frac{1}{16 A^{3}} \frac{\partial}{\partial Q} W_{2}-\frac{1}{8 A} \frac{\partial}{\partial Q} W_{1}, \\
\frac{\partial E}{\partial v} & =\left(\frac{1}{8 A^{2}} W_{1}-\frac{3}{16 A^{4}} W_{2}\right) \nabla A+\left(\frac{1}{16 A^{3}} \frac{\partial}{\partial v} W_{2}\right)
\end{aligned}
$$

It follows:

$$
\begin{aligned}
\left(\frac{\partial E}{\partial v}\right)_{p}= & -\frac{n \times e_{p}}{4 A}\left(\frac{\operatorname{Det}(Q)}{8 A^{2}}-\frac{3 \operatorname{Tr}(Q)^{2}}{16 A^{4}}\right)+ \\
& \frac{1}{8 A^{3}} \operatorname{Tr}(Q)\left(Q_{p}\left(e_{j}-e_{k}\right)+e_{p}\left(Q_{j}-Q_{k}\right)\right) .
\end{aligned}
$$




\section{A. Derivatives}

\section{Hessian}

The hessian is:

$$
\begin{aligned}
H=-\nabla^{2} E= & -\left(\frac{\partial^{2} Q}{\partial v^{2}} \frac{\partial E}{\partial Q}+\frac{\partial Q}{\partial v} \frac{\partial^{2} E}{\partial Q^{2}} \frac{\partial Q}{\partial v}\right)-\left(\frac{\partial Q}{\partial v} \frac{\partial^{2} E}{\partial v \partial Q}+\frac{\partial^{2} E}{\partial Q \partial v} \frac{\partial Q}{\partial v}\right)-\frac{\partial^{2} E}{\partial v^{2}} \\
= & H_{C S T}-\left(\frac{\partial Q}{\partial v} \frac{\partial^{2} E}{\partial v \partial Q}+\frac{\partial^{2} E}{\partial Q \partial v} \frac{\partial Q}{\partial v}\right)-\frac{\partial^{2} E}{\partial v^{2}} \\
\frac{\partial^{2} E}{\partial v^{2}}= & \nabla A^{T} \nabla A\left(\frac{3}{4 A^{5}} W_{2}-\frac{1}{4 A^{3}} W_{1}\right)+\nabla^{2} A\left(\frac{1}{8 A^{2}} W_{1}-\frac{3}{16 A^{4}} W_{2}\right) \\
& -\frac{3}{16 A^{4}}\left(\nabla A^{T} \frac{\partial}{\partial v} W_{2}+\frac{\partial}{\partial v} W_{2} \nabla A^{T}\right)+\left(\frac{1}{16 A^{3}} \frac{\partial^{2}}{\partial v^{2}} W_{2}\right) \\
\frac{\partial^{2} E}{\partial Q \partial v}+\frac{\partial^{2} E}{\partial v \partial Q}= & \frac{1}{8 A^{2}}\left(\nabla A \frac{\partial W_{1}}{\partial Q}+\frac{\partial W_{1}}{\partial Q} \nabla A\right)-\frac{3}{16 A^{4}}\left(\nabla A \frac{\partial W_{2}}{\partial Q}+\frac{\partial W_{2}}{\partial Q} \nabla A\right) \\
& +\frac{1}{16 A^{3}}\left(\frac{\partial^{2} W_{2}}{\partial v \partial Q}+\frac{\partial^{2} W_{2}}{\partial Q \partial v}\right) .
\end{aligned}
$$

\section{Mixed Hessian}

$$
\begin{aligned}
\left(\frac{\partial Q_{i}}{\partial v}\right)_{p} & =2 e_{i}\left(\delta_{p k}-\delta_{p j}\right), \\
\left(\frac{\partial Q_{i}}{\partial \bar{v}}\right)_{p} & =-2 \bar{e}_{i}\left(\delta_{p k}-\delta_{p j}\right), \\
\left(\frac{\partial^{2} Q_{i}}{\partial v^{2}}\right)_{p q} & =-\left(\frac{\partial^{2} Q_{i}}{\partial v^{2}}\right)_{p q} \\
& =2\left(\delta_{q k}-\delta_{q j}\right)\left(\delta_{p k}-\delta_{p j}\right), \\
\left(\frac{\partial^{2} Q_{i}}{\partial \bar{v} \partial)_{p q}}\right. & =0, \\
F & =-\frac{\partial Q}{\partial \bar{v}} \frac{\partial E}{\partial Q}-\frac{\partial E}{\partial \bar{v}}=-\frac{\partial Q}{\partial \bar{v}} \frac{\partial E}{\partial Q}=\bar{F}_{C S T}, \\
H & =-\frac{\partial}{\partial v}\left(\frac{\partial Q}{\partial \bar{v}} \frac{\partial E}{\partial Q}\right)=-\frac{\partial Q}{\partial \bar{v}} \frac{\partial^{2} E}{\partial Q^{2}} \frac{\partial Q}{\partial v}-\frac{\partial Q}{\partial \bar{v}} \frac{\partial^{2} E}{\partial Q \partial v}, \\
\left(\frac{\partial^{2} E}{\partial Q^{2}}\right)_{p q} & =\frac{1}{8 A^{3}}\left(e_{j} \cdot e_{k}\right)\left(e_{r} \cdot e_{s}\right)-\frac{1}{4 A}\left(\delta_{j q}+\delta_{k q}-\delta_{q p}\right) .
\end{aligned}
$$

\section{Efficiency}

For a more efficient assembly, we need to directly evaluate the following quantities:

$$
\left(\frac{\partial Q}{\partial v} \frac{\partial^{2} W_{2}}{\partial v \partial Q}\right)_{p q}=\left(\sum_{i} \frac{\partial Q_{i}}{\partial v} \frac{\partial^{2} W_{2}}{\partial v \partial Q_{i}}\right)_{p q}=\sum_{i} 2 e_{i}\left(\delta_{p k}-\delta_{p j}\right)\left(\frac{\partial^{2} W_{2}}{\partial v \partial Q_{i}}\right)_{q}
$$




\section{Bending derivatives}

We here present the derivation of the DS energy with respect of the undeformed vertices, required in order to compute the gradients and the Hessians of r-adaptivity.

\section{Edge Length}

Let us denote with $L=\left\|p_{2}-p_{1}\right\|^{2}=\|e\|^{2}$ the squared hinge length, with $p$ and $q$ the hinge and flap vertices, respectively. We have:

$$
\begin{aligned}
(\nabla L)_{p_{1}} & =-(\nabla L)_{p_{2}}=-2\left(p_{2}-p_{1}\right)=-2 e \\
(\nabla L)_{q_{1}} & =(\nabla L)_{q_{2}}=0 \\
\left(\nabla^{2} L\right)_{p_{i} p_{i}} & =2, \quad\left(\nabla^{2} L\right)_{p_{i} p_{j}}=-2 .
\end{aligned}
$$

\section{Area}

Let us denote with $A=\left\|n_{1}\right\|+\left\|n_{2}\right\|$ the area associated to a hinge, with $p$ and $q$ the hinge and flap vertices, respectively. We have:

$$
\begin{aligned}
(\nabla A)_{p_{1}}=\left(\nabla\left\|n_{1}\right\|+\nabla\left\|n_{2}\right\|\right)_{p_{1}} & =\frac{n_{1} \times\left(p_{2}-q_{1}\right)}{\left\|n_{1}\right\|}+\frac{n_{2} \times\left(p_{2}-q_{2}\right)}{\left\|n_{2}\right\|}, \\
(\nabla A)_{p_{2}}=\left(\nabla\left\|n_{1}\right\|+\nabla\left\|n_{2}\right\|\right)_{p_{2}} & =\frac{n_{1} \times\left(p_{1}-q_{1}\right)}{\left\|n_{1}\right\|}+\frac{n_{2} \times\left(p_{1}-q_{2}\right)}{\left\|n_{2}\right\|}, \\
(\nabla A)_{q_{1}}=\left(\nabla\left\|n_{1}\right\|\right)_{q_{1}} & =\frac{n_{1} \times e}{\left\|n_{1}\right\|}, \\
(\nabla A)_{q_{2}}=\left(\nabla\left\|n_{2}\right\|\right)_{q_{2}} & =\frac{n_{2} \times e}{\left\|n_{2}\right\|}, \\
\left(\nabla^{2} A\right)_{p_{i} p_{j}} & =\left(\nabla^{2}\left\|n_{1}\right\|+\nabla^{2}\left\|n_{2}\right\|\right)_{p_{i} p_{j}}, \\
\left(\nabla^{2} A\right)_{q_{i} q_{j}} & =\left(\nabla^{2}\left\|n_{i}\right\|\right)_{q_{i} q_{j}}, \\
\left(\nabla^{2} A\right)_{q_{i} p_{j}} & =\left(\nabla^{2}\left\|n_{i}\right\|\right)_{q_{i} p_{j}}
\end{aligned}
$$

For the second derivatives, consider a single triangle with normal $n$, vertices $v_{i}, v_{k}, v_{j}$, and edges $e_{i}, e_{j}, e_{k}$.

$$
\begin{aligned}
\left(\nabla^{2}\|n\|\right)_{v_{i} v_{j}} & =\left(\nabla\left(\frac{n \times e_{i}}{\|n\|}\right)\right)_{v_{j}}=\frac{(\nabla(n \times e))_{v_{j}}}{\|n\|}-\frac{(\nabla\|n\|)_{v_{i}}(\nabla\|n\|)_{v_{j}}}{\|n\|}, \\
\left(\nabla\left(n \times e_{p}\right)\right)_{q} & =e_{p} \cdot e_{q} \delta_{p q}^{3}+\left(e_{p} \otimes e_{q}-2 e_{q} \otimes e_{p}\right), \quad q \geq p,
\end{aligned}
$$

where $\delta_{p q}^{3}$ is a $3 \times 3$ matrix, having the Kronecker delta $\delta_{p q}$ on its diagonal.

\section{Energy}

On a single edge, the energy is:

$$
E=3 \frac{L}{A} \theta^{2}
$$




\section{A. Derivatives}

\section{Force}

The gradient is:

$$
\begin{aligned}
\nabla E & =\nabla\left(\frac{3}{A}\right) L \theta^{2}+\frac{3}{A} \nabla\left(L \theta^{2}\right)=-3 \frac{\nabla A}{A^{2}} L \theta^{2}+\frac{3}{A} \nabla\left(L \theta^{2}\right) . \\
\nabla\left(L \theta^{2}\right) & =\nabla L \theta^{2}+2 L \theta \nabla \theta .
\end{aligned}
$$

The forces are obtained by augmenting the standard DS vector:

$$
\begin{aligned}
F_{D S} & =-\frac{6 L \theta}{A} \nabla \theta, \\
F=-\nabla E & =F_{D S}+3 \frac{\nabla A}{A^{2}} L \theta^{2}-3 \frac{\theta^{2}}{A} \nabla L .
\end{aligned}
$$

\section{Hessian}

The second derivative is:

$$
\begin{aligned}
\nabla^{2} E= & \nabla^{2}\left(\frac{3}{A}\right) L \theta^{2}+\nabla\left(\frac{3}{A}\right) \nabla\left(L \theta^{2}\right)+\nabla\left(L \theta^{2}\right) \nabla\left(\frac{3}{A}\right)+\frac{3}{A} \nabla^{2}\left(L \theta^{2}\right) \\
= & L \theta^{2}\left(\frac{6}{A^{3}} \nabla A^{T} \nabla A-\frac{3}{A^{2}} \nabla^{2} A\right)- \\
& \frac{3}{A^{2}}\left(\nabla A^{T} \nabla\left(L \theta^{2}\right)+\nabla\left(L \theta^{2}\right)^{T} \nabla A\right)+\frac{3}{A} \nabla^{2}\left(L \theta^{2}\right) . \\
\nabla^{2}\left(L \theta^{2}\right)= & \nabla^{2} L \theta^{2}+2 \theta(\nabla L \nabla \theta+\nabla \theta \nabla L)+2 L \nabla \theta \nabla \theta+2 L \theta \nabla^{2} \theta .
\end{aligned}
$$

The Hessian is obtained by augmenting the standard DS matrix:

$$
\begin{aligned}
H_{D S}= & -\frac{6 L}{A}\left(\nabla \theta \nabla \theta+\theta \nabla^{2} \theta\right), \\
H=-\nabla^{2} E= & H_{D S}-2 \frac{E}{A^{2}} \nabla A^{T} \nabla A+\frac{E}{A} \nabla^{2} A+3 \frac{\theta^{2}}{A^{2}}\left(\nabla A^{T} \nabla L+\nabla L^{T} \nabla A\right) \\
& +6 \frac{L \theta}{A^{2}}\left(\nabla A^{T} \nabla \theta+\nabla \theta^{T} \nabla A\right)-3 \frac{\theta^{2}}{A} \nabla^{2} L-6 \frac{\theta}{A}(\nabla L \nabla \theta+\nabla \theta \nabla L) .
\end{aligned}
$$




\section{B. Implementation}

We have implemented the contend of this thesis in a $\mathrm{C}++$ library called MESHOPT. Here we present an overview of its structure and of our contribution. An illustration is given in figure B.1.

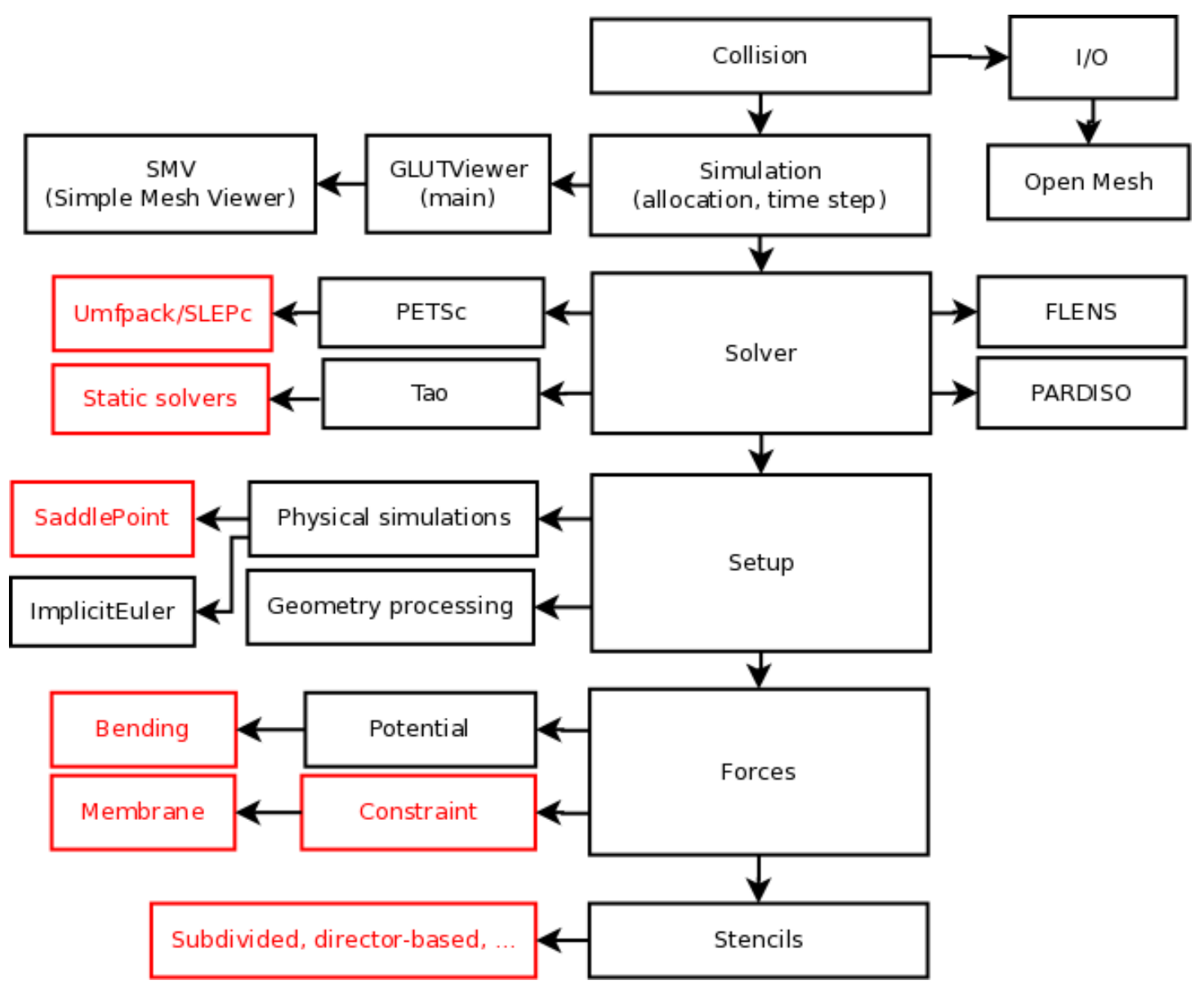

Figure B.1.: Structure of Meshopt class tree. The red blocks were created or modified during this thesis. 


\section{B. Implementation}

\section{Setup classes}

The original SetupProblem base abstract classes was intended to find its stationary points of the Lagrangian

$$
E(q, \dot{q})=E_{k i n}(\dot{q})-E_{p o t}(q),
$$

where $q \in \Re^{n}$ is the state variable. In particular, DynAmicProblem solves

$$
M \ddot{q}-\nabla E_{p o t}(q)=0,
$$

where $M$ is the lumped mass matrix.

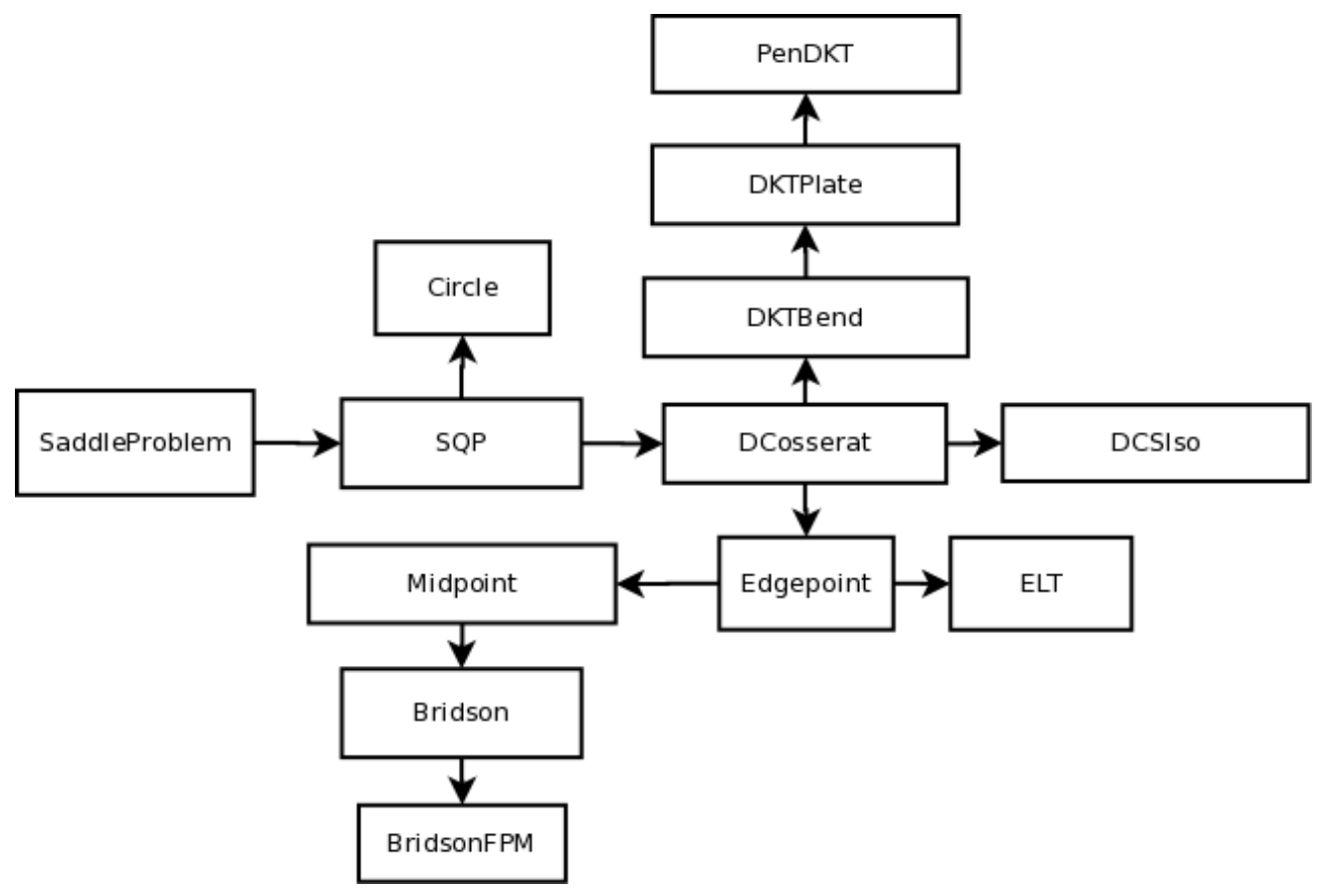

Figure B.2.: Structure of the setup class tree.

In our new implementation shown in figure B.2, we are given the vector $c(q) \in \Re^{m}$ of prescribed constraints, so the SADDLEPROBLEM Lagrangian is

$$
L(q, \dot{q}, \sigma)=E(q, \dot{q})-\sigma^{T} c(q)+\mu\|c(q)\|^{2}+\frac{\gamma}{2}\|\sigma\|^{2},
$$

where $\sigma \in \Re^{m}$ are Lagrange multipliers and $\gamma, \mu \in \Re^{2}$ are stabilization parameters. Typically $\gamma \approx 10^{-9}$ if $\nabla c(q)^{T}$ is not full rank. Note that in particular

$$
L(q, \dot{q}, \sigma)=E(q, \dot{q})+(\mu c(q)-\sigma)^{T} c(q)+\frac{\gamma}{2}\|\sigma\|^{2} .
$$


Assuming the static case for simplicity, corresponding Euler-Lagrange equations are

$$
\begin{aligned}
F(q, \sigma) & =0, \\
c(q) & =\gamma \sigma .
\end{aligned}
$$

whose Newton iteration is

$$
\left[\begin{array}{cc}
(\mu c-\sigma)^{T} \nabla^{2} c+\mu \nabla c^{T} \nabla c & \nabla c^{T} \\
\nabla c & -\gamma I d
\end{array}\right]\left[\begin{array}{c}
d q \\
d \sigma
\end{array}\right]=\left[\begin{array}{c}
F(q, \sigma) \\
c(q)
\end{array}\right] .
$$

Remark 1 . The variables $d q$ and $d \sigma$ are the corrections of the guesses $q$ and $\sigma$. Typically, $q$ denotes a displacement, which has not to be confused with the meaning of $d q$.

Remark 2 . In the dynamic case, the positional variable can be expressed using an implicit scheme $q_{n+1}=q_{n}+h \dot{q}_{n+1}$, where $h$ is the time step size. Then, for $\mu=0$ the Newton iteration becomes

$$
\left[\begin{array}{cc}
M-h^{2}\left(\nabla^{2} E_{p o t}\left(q_{n}\right)+\sigma_{n} \nabla^{2} c\left(q_{n}\right)\right) & -h \nabla c\left(q_{n}\right) \\
-h \nabla c\left(q_{n}\right)^{T} & -\gamma I d
\end{array}\right]\left[\begin{array}{c}
d \dot{q} \\
d \sigma
\end{array}\right]=\left[\begin{array}{c}
-h F\left(\dot{q}_{n}, \sigma_{n}\right) \\
-c\left(q_{n}\right)
\end{array}\right]
$$

The role of the remaining classes shown in figure B.2 is briefly explained as follows:

- SQP: interfaces SADDLEPROBLEM with PETSc nonlinear solver.

- DCosserat: discretized bending energies using directors.

- DKTBEnd: discretizes the bending energy using DKT elements.

- DKtPlate: discretizes the membrane energy using DKT elements.

- PENDKT: selects between penalty and multipliers formulations.

- Midpoint: handles methods based on edge midpoints.

- Bridson: implements Bridson's paper [17].

- Edgepoint: uses edgepoints.

- RADAPTIVITY: uses r-adaptivity.

- CirClE: handles perimeter-preserving elements.

\section{Solver classes}

We provide the following nonlinear solvers:

- PETScSolver: base abstract class.

- SimplePETScSolver: basic Newton solver for SAddleProblem.

- IncrLoadNewton: Newton with incremental loading, to tackle the issue of providing a good initial guess. 


\section{B. Implementation}

- ArcLengthnewton: incremental loading with arc length continuation, to handle bifurcations.

- InCRPenNeWton: constraint enforcement using an incremental penalty method.

- AugmentedLag: combining penalty and duality to avoid ill-conditioning using Lancelot [36].

- BARRIER: barrier method for inequality constraints.

- RectPetScNewton: Gauss-Newton for over- and under-constrained systems using least squares.

- SingularSLEPcNewton: singular Jacobian handled by using a Singular Value Decomposition.

- FastProjection: abstract implementation of [23].

- ShellPETScSolver: matrix-free Newton solver.

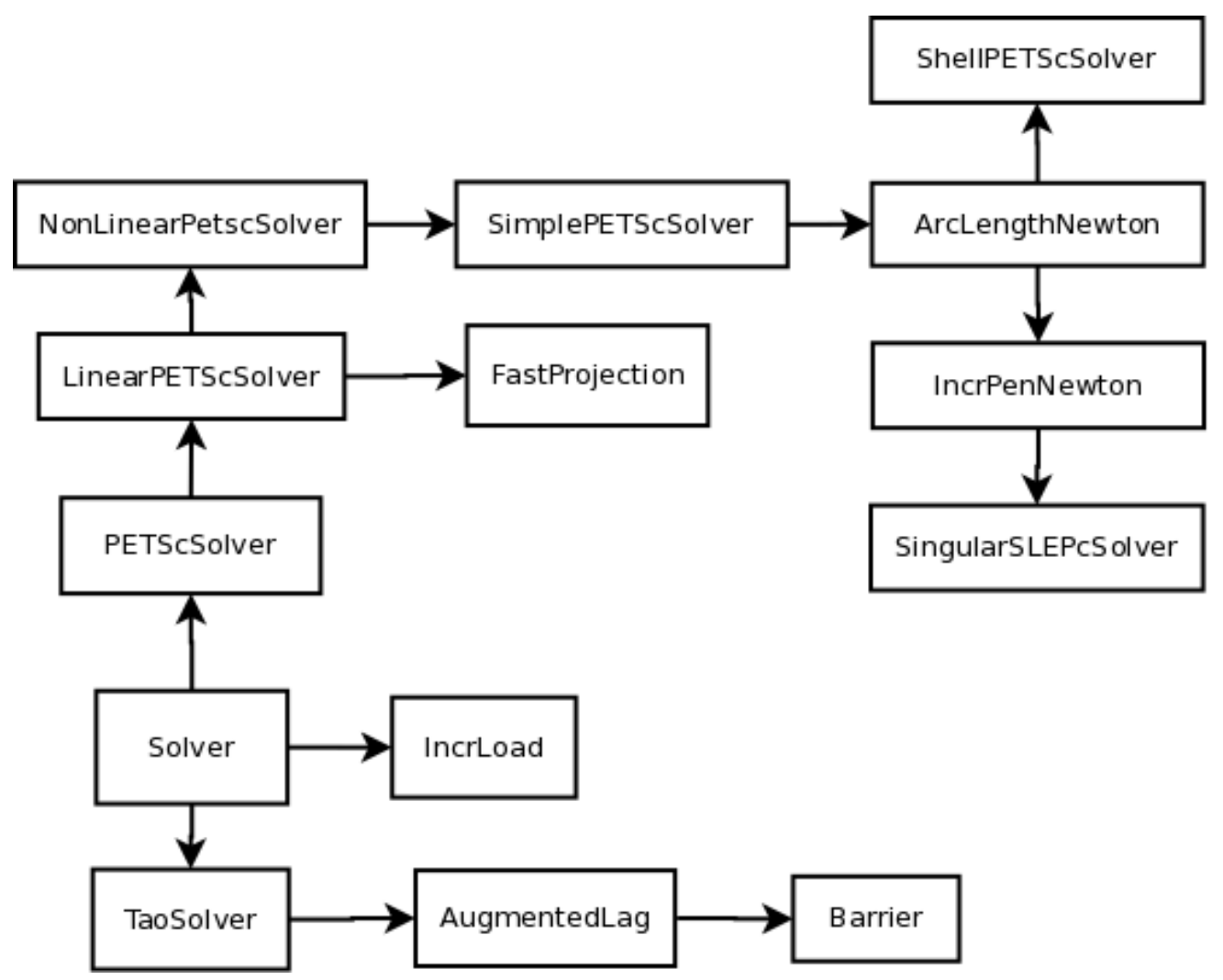

Figure B.3.: Structure of the solver class tree. 


\section{Acknowledgements}

There are three groups of people I wish to thank. First and foremost, I wish to thank Max for his mentoring, which helped me to evolve from a newly-minted engineer to an (almost) independent young scientist.

Secondly, I wish to thank the people who gave me any sort of technical help. Gert Lube and Clarisse for having read my thesis before the submission, providing me with many useful comments. Lars for his tips regarding the bureaucratic matters. Eitan Grinspun, partially for his help with obtaining the frustrating negative results of Chapter 5 , but mainly for our great trip to Paris. Joachim Linn for the lengthy but insightful discussions, which eventually led me to formulate the bending model presented in Chapter 6 .

Last but certainly not least, I wish to thank the people who gave me moral support. Alessandro and Marco for having participated in the creation of a virtual office, spanning $900 \mathrm{~km}$ from Milan to Göttingen, passing through Lugano, in which our online chats accompany everyday our work. I wish them all the best for their exams, too. My parents for the encouragement during the difficult moments. But above all of them, I thank Iliana, because I am certain that without her I would not have managed to reach the end of this journey. 



\section{Bibliography}

[1] Arnold, D., And Brezzi, F. Locking-free finite element methods for shells. Math. Comput. 66 (1997), 1-14.

[2] Arnold, D., Brezzi, F., And Marini, L. A family of discontinuous galerkin finite elements for the reissner-mindlin plate. Journal of Scientific Computing 22, 1 (2005), $25-45$.

[3] Arnold, D., And Falk, R. A uniformly accurate finite element method for the reissner-mindlin plate. SIAM J. Numer. Anal. 26, 6 (1989), 1276-1290.

[4] Audoly, B., And Pomeau, Y. Elasticity and geometry: from hair curls to the nonlinear response of shells. Oxford University Press, 2010.

[5] Bathe, K., And Chapelle, D. The Finite Element Analysis of Shells: Fundamentals, 1 ed. Springer, 2003.

[6] Batoz, J.-L., BAthe, K.-J., AND Ho, L.-W. A study of three-node triangular plate bending elements. International Journal for Numerical Methods in Engineering 15, 12 (1980), 1171-1812.

[7] Benson, D., Bazilevs, Y., Hsu, M., and Hughes, T. Isogeometric shell analysis: the reissner-mindlin shell. Computer Methods in Applied Mechanics and Engineering 199, 58 (2010), $276-289$.

[8] BerdichevskiI, V. L. Variational principles of continuum mechanics. Springer, 2009.

[9] Bergou, M., Wardetzky, M., Harmon, D., Zorin, D., and Grinspun, E. A quadratic bending model for inextensible surfaces. In Proceedings of the fourth Eurographics symposium on Geometry processing (Aire-la-Ville, Switzerland, Switzerland, 2006), SGP '06, Eurographics Association, pp. 227-230.

[10] Bergou, M., Wardetzky, M., Robinson, S., Audoly, B., and Grinspun, E. Discrete elastic rods. In ACM Siggraph Asia 2008 courses (New York, NY, USA, 2008), SIGGRAPH Asia '08, ACM, pp. 14:1-14:12.

[11] Boffi, D., and Gastaldi, L. Mixed finite elements, compatibility conditions, and applications. Springer, Berlin, 2008.

[12] Braess, D. Finite Elemente, 1 ed. Springer-Verlag GmbH, 2007. 
Bibliography

[13] Brezzi, F., and Fortin, M. Mixed and hybrid finite element methods. SpringerVerlag New York, Inc., New York, NY, USA, 1991.

[14] Chapelle, D., And Bathe, K. J. Fundamental considerations for the finite element analysis of shell structures. Computers and Structures 66, 1 (1998), 19 36.

[15] Choi, D., Palma, F., Sanchez-Palencia, E., and Vilariño, M. Membrane locking in the finite element computation of very thin elastic shells. R.A.I.R.O. 32, 2 (1998).

[16] Cirak, F., Ortiz, M., And Schröder, P. Subdivision surfaces: A new paradigm for thin-shell finite-element analysis. International Journal for Numerical Methods in Engineering 47 (2000), 2039-2072.

[17] English, E., And Bridson, R. Animating developable surfaces using nonconforming elements. ACM Trans. Graph. 27 (August 2008), 66:1-66:5.

[18] FALK, R. Nonconforming finite element methods for the equations of linear elasticity. Math. Comp. 57, 196 (1991), 529-550.

[19] Felippa, C. A. Refined finite element analysis of linear and nonlinear twodimensional structures. PhD thesis, University of California, 1972.

[20] Felippa, C. A. A study of optimal membrane triangles with drilling freedoms. Comput. Methods Appl. Mech. Eng. 192, 16-18 (April 2003), 2125-2168.

[21] Gardsback, M., And Tibert, G. A comparison of rotation-free triangular shell elements for unstructured meshes. Computer Methods in Applied Mechanics and Engineering 196 (Nov. 2007), 5001-5015.

[22] Gingold, Y., Secord, A., Han, J. Y., Grinspun, E., and Zorin, D. A Discrete Model for Inelastic Deformation of Thin Shells. Tech. rep., Courant Institute of Mathematical Sciences, New York University, Aug 2004.

[23] Goldenthal, R., Harmon, D., Fattal, R., Bercovier, M., and Grinspun, E. Efficient simulation of inextensible cloth. ACM Trans. Graph. 26 (July 2007).

[24] Goss, V., van der Heijden, G., Thompson, J., and Neukirch, S. Experiments on snap buckling, hysteresis and loop formation in twisted rods. Experimental Mechanics 45 (2005), 101-111. 10.1007/BF02428182.

[25] Grinspun, E., Gingold, Y., Reisman, J., and Zorin, D. Computing discrete shape operators on general meshes. Eurographics (Computer Graphics Forum) 25 (2006), 547-556.

[26] Grinspun, E., Hirani, A. N., Desbrun, M., And Schröder, P. Discrete shells. In Proceedings of the 2003 ACM SIGGRAPH/Eurographics symposium on 
Computer animation (Aire-la-Ville, Switzerland, Switzerland, 2003), SCA '03, Eurographics Association, pp. 62-67.

[27] Hardesty, S. S. Optimization of shell structure acoustics. PhD thesis, Rice University, 2010.

[28] Hirani, A. N. Discrete Exterior Calculus. PhD thesis, California Institute of Technology, 2005.

[29] Hoffman, J., And Johnson, C. Blow up of incompressible euler equations. BIT Numerical Mathematics 48 (2008), 285-307.

[30] Hubert, J. S. Coques élastiques minces : propriétés asymptotiques. Masson, Paris, 1997.

[31] Jarasjarungkiat, A., Wüchner, R., and Bletzinger, K.-U. Efficient subgrid scale modeling of membrane wrinkling by a projection method. Computer Methods in Applied Mechanics and Engineering 198 (2009), 1097 - 1116.

[32] Levien, R. The Elastica: a Mathematical History. EECS Technical Reports. University of California, Berkeley, California, 2008.

[33] Love, A. E. A treatise on the mathematical theory of elasticity, 4th ed. ed. Dover Publications, New York, 1944.

[34] Mosler, J. A novel variational algorithmic formulation for wrinkling at finite strains based on energy minimization: Application to mesh adaption. Computer Methods in Applied Mechanics and Engineering 197, 9-12 (2008), 1131 - 1146.

[35] Mosler, J., And Ortiz, M. On the numerical implementation of variational arbitrary lagrangian-eulerian (vale) formulations. International Journal for Numerical Methods in Engineering 67, 9 (2006), 1272-1289.

[36] Nocedal, J., And Wright, S. Numerical Optimization. Springer, 2000.

[37] OÑate, E., Zarate, F., And Flores, F. A simple triangular element for thick and thin plate and shell analysis. International Journal for Numerical Methods in Engineering 37, 15 (1994), 2569-2582.

[38] O'NeIll, B. Elementary Differential Geometry, Revised 2nd Edition, 2 ed. Academic Press, 2006.

[39] Papadopoulos, P., and Taylor, R. L. A triangular element based on reissnermindlin plate theory. International Journal for Numerical Methods in Engineering 30, 5 (1990), 1029-1049.

[40] Paris, I. Robustesse des Éléments Finis Triangulaires de Coque. Ph.d. thesis, Université Pierre et Marie Curie - Paris VI, Nov. 2006. 
Bibliography

[41] Pavlov, D., Mullen, P., Tong, Y., Kanso, E., Marsden, J. E., and DesBRUn, M. Structure-preserving discretization of incompressible fluids. Physica D: Nonlinear Phenomena (2011).

[42] Phaal, R., and Calladine, C. R. A simple class of finite elements for plate and shell problems. I - Elements for beams and thin flat plates. II - An element for thin shells, with only translational degrees of freedom. International Journal for Numerical Methods in Engineering 35 (Sept. 1992), 955-977.

[43] Pottmann, H., And Wallner, J. Computational Line Geometry. Mathematics and Visualization. Springer, Dordrecht, 2010.

[44] Reddy, J. N. An introduction to nonlinear finite element analysis. Oxford University Press, Oxford, 2004.

[45] Rossi, R., Lazzari, M., Vitaliani, R., and OÑate, E. Simulation of lightweight membrane structures by wrinkling model. International Journal for Numerical Methods in Engineering 62, 15 (2005), 2127-2153.

[46] Scherer, M., Denzer, R., And Steinmann, P. On a solution strategy for energy-based mesh optimization in finite hyperelastostatics. Computer Methods in Applied Mechanics and Engineering 197, 6-8 (2008), $609-622$.

[47] Scholz, R. A mixed method for 4th order problems using linear finite elements. R.A.I.R.O. Numerical Analysis 12 (1978), 85-90.

[48] Simo, J. C., And Armero, F. Geometrically non-linear enhanced strain mixed methods and the method of incompatible modes. International Journal for Numerical Methods in Engineering 33, 7 (1449 1992), 1413.

[49] Simo, J. C., And Fox, D. D. On stress resultant geometrically exact shell model. part i: formulation and optimal parametrization. Comput. Methods Appl. Mech. Eng. 72 (March 1989), 267-304.

[50] Stern, A., Tong, Y., Desbrun, M., and Marsden, J. E. Variational Integrators for Maxwell's Equations with Sources. ArXiv e-prints (Mar. 2008).

[51] Stolarski, H., Belytschko, T., Carpenter, N., and Kennedy, J. M. A simple triangular curved shell element. International Journal for Computer-Aided Engineering and Software 1, 3 (1984), 210-218.

[52] Sze, K. Y., Liu, X. H., AND Lo, S. H. Popular benchmark problems for geometric nonlinear analysis of shells. Finite Elem. Anal. Des. 40 (July 2004), 1551-1569.

[53] Thoutireddy, P., AND Ortiz, M. A variational r-adaption and shapeoptimization method for finite-deformation elasticity. International Journal for Numerical Methods in Engineering 61, 1 (2004), 1-21. 
[54] Timoshenko, S., And Woinowsky-Kreiger. Theory of Plates and Shells (McGraw-Hill Classic Textbook Reissue Series), 2nd ed. McGraw Hill Higher Education, 1964.

[55] Trimarchi, D., Turnock, S. R., and Taunton, D. J. The use of shell elements to capture sail wrinkles, and their influence on aerodynamic loads. In Proceedings of the Second International Conference on Innovation in High Performance Sailing Yachts, Lorient, France (2010).

[56] Van Keulen, F., And BooiJ, J. Refined consistent formulation of a curved triangular finite rotation shell element. International Journal for Numerical Methods in Engineering 39, 16 (1996), 2803-2820.

[57] Zielonka, M. G., Ortiz, M., and Marsden, J. E. Variational r-adaption in elastodynamics. International Journal for Numerical Methods in Engineering 74, 7 (2008), 1162-1197.

[58] Zienkiewicz, O., and Taylor, R. The Finite Element Method for Solid and Structural Mechanics, Sixth Edition, 6 ed. Butterworth-Heinemann, 2005. 



\section{Curriculum Vitæ}

\section{Personal}

$\begin{array}{ll}\text { Name } & \text { Alessio Quaglino } \\ \text { Date of birth } & \text { April 25th, 1983 } \\ \text { Place of birth } & \text { Cantù(CO), Italy } \\ \text { Citizenship } & \text { Italian }\end{array}$

\section{Professional}

since 11/2008 Research Assistant

Discrete Differential Geometry Lab

Georg-August-Universität Göttingen, Germany

2-3/2012 Research Intern

Fraunhofer ITWM, Kaiserslautern, Germany

8-12/2007 Physics Programmer

Craft Animations AB, Gothenburg, Sweden

\section{Education}

2008 M.Sc., Engineering Mathematics

Politecnico di Milano, Milan, Italy

2007 M.Sc., Engineering Physics

Royal Institute of Technology, Stockholm, Sweden

2005 B.Sc., Engineering Mathematics

Politecnico di Milano, Milan, Italy 